\title{
DESENVOLVIMENTO DE UMA BIBLIOTECA DE OBJETOS PARA USO EM PROGRAMAS DE ESTATÍSTICA E DE MATEMÁTICA
}

\section{AMAURI DE ALMEDA MACHADO}

Engenheiro Agrônomo

Orientador: Prof. Dr. CASSIO ROBERTO DE MELO GODOI

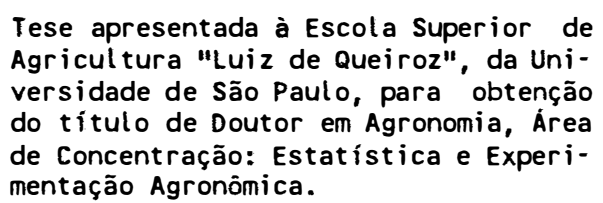

P I R A C I C A B A

Estado de São Paulo - Brasil

Junho - 1993 
Ficha catalografica preparada pela Seça de Livros da Divisán de Biblioteca e Documentaça - PClQuSP

Machado, Amauri de Almeida

M149d Desenvolvimento de uma biblioteca de objetos para uso em programas de matematica e de estatistica. Piracicaba, 1993. $202 \mathrm{p}$.

Tese - EsalQ

Bibliografia.

1. Estatistica - Informática Z. Informática agrico1 a 3. Matemática - Informatica 4. Programa de computa dor 5. Frogramaço orientada a objeto I. Escola Superior de Agricultura Luiz de Queiroz, Firacicaba

CDD 001.8424

519.50285 


\section{DESENVOLVIMENTO DE UMA BIBLIOTECA DE OBJETOS PARA}

USO EM PROGRAMAS DE ESTATÍSTICA E DE MATEMÁTICA

\section{AMAURI DE ALMEIDA MACHADO}

Aprovada em 12/08/93

Comissão julgadora

Prof. Dr. Cassio Roberto de Melo Godoi ESALQ-USP

Prof. Dr. Pedro Valentim Marques ESALQ-USP

Prof. Dr. João Gilberto Correa da Silva UFPEL/Pelotas

Prof. Dr. José Carlos Barbosa

UNESP/Jaboticabal

Prof $^{\text {a }}$ Dr $^{\text {a }}$ Sheila Maria Zambelo Pinho UNESP/Botucatu

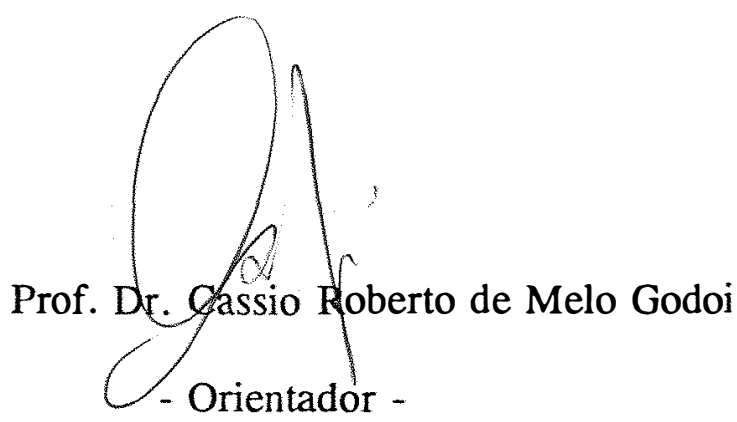


Aos meus pais (in memorian), ofereço

À Dida, Carolina e Juliano, dedico com todo amor. 


\section{AGRADECIMENTOS}

Ao Professor Dr. Cassio Roberto de Melo Godoi, pela orientação e pela sempre profícua troca de idéias.

Aos professores do Departamento de Matemática e Estatística, pelos ensinamentos e pelo convívio cordial.

Aos professores Clarice Borges Demétrio e Décio Barbin, pela amizade.

A todos os funcionários, em particular à Rosa e à Solange, pelo apoio.

Ao grąnde amigo Luis Eloi (in memorian) pelo convívio enquanto o destino

permitiu.

Ao Professor Paulo Silveira Júnior, grande amigo e companheiro, pelo apoio irrestrito.

Aos Professores Élio Paulo Zonta, João Baptista da Silva e João Gilberto Corrêa da Silva, pelo apoio e amizade.

À Maria Izalina Ferreira Alves, uma pessoa rara.

Aos meus colegas de curso, em particular ao amigo Paulo Cecon, pelo companheirismo e pela confiança em mim depositada.

Aos meus irmãos que além de familiares são amigos, pelo apoio incondicional.

À Universidade Federal de Pelotas, em particular ao Departamento de Matemática, Estatística e Computação, pela oportunidade de realizar o curso.

Ao Programa PICD da CAPES, pela bolsa concedida.

A todos os que direta ou indiretamente colaboraram com esta importante etapa de minha vida. 


\section{ÍNDICE}

Página

LISTA DE TABELAS ............................... vi

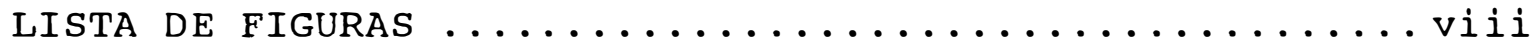

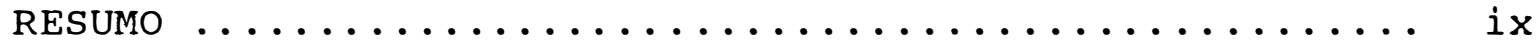

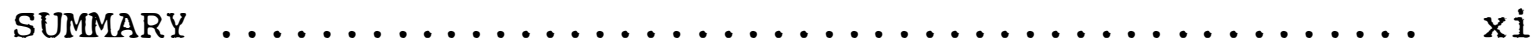

1. INTRODUÇÃO $\ldots \ldots \ldots \ldots \ldots \ldots \ldots \ldots \ldots \ldots \ldots \ldots \ldots \ldots \ldots \ldots$

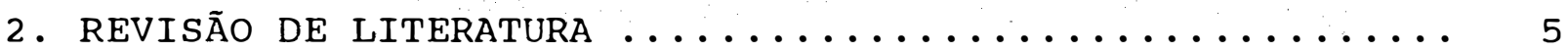

2.1. Programação orientada a objetos (POO) e o Pascal .. 5

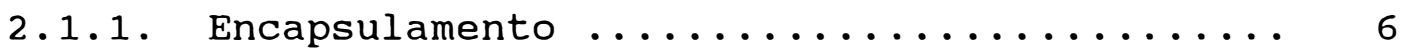

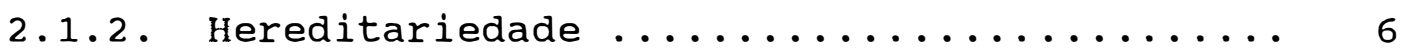

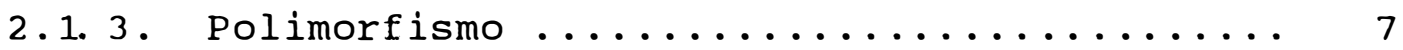

2.2. Alguns Algoritmos Básicos ................. 8

2.2.1. Avaliação de expressōes ............... 8

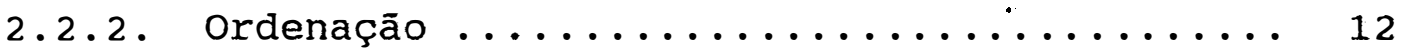

2.2.3. Geração de índices .................. 14

2.2.4. O método de Gauss-Jordan ............. 15

2.2.5. A decomposição LU e relacionados ......... 16

2.2.6. O método de cholesky e relacionados ...... 19

2.2.7. O operador SWEEP e relacionados ......... 21

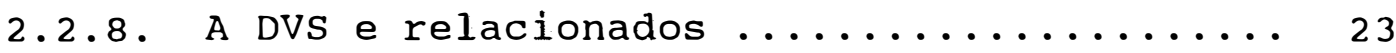

2.2.9. A decomposição ortogonal e relacionados ... 26

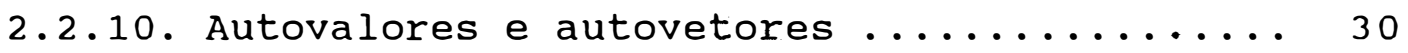

2.2.11. A geração de variáveis aleatórias ....... 32

2.2.12. Probabilidades e pontos percentuais ...... 35

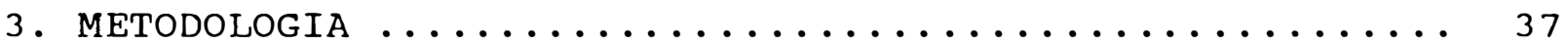

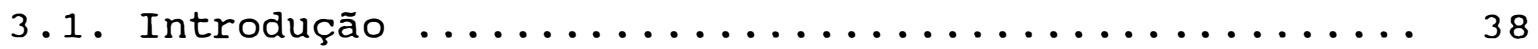

3.2. A Programação Dirigida a Eventos .............. 38

3.3. A Biblioteca de Objetos do Turbo Vision ........ 39

3.3.1. Objetos primitivos ................. 40

3.3.2. Objetos de interface ................. 41

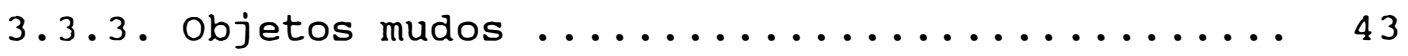

3.3 .4 . outros objetos .................. 46 
3.3.5. Convenções de nome ................... 47

3.4. A Navegação no Turbo Vision ................ 48

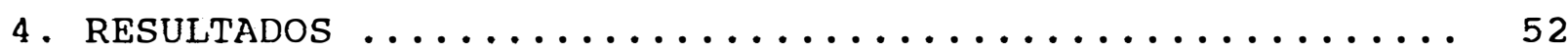

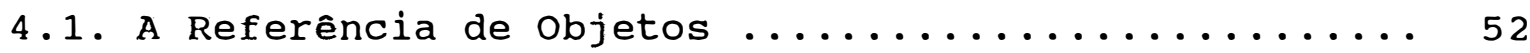

4.1 .1$. Arquivos de dados ................... 53

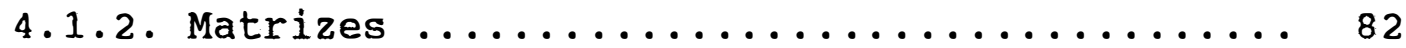

4.1.3. Geração de variáveis aleatórias ......... 105

4.2. Referência das Rotinas Externas à Biblioteca de

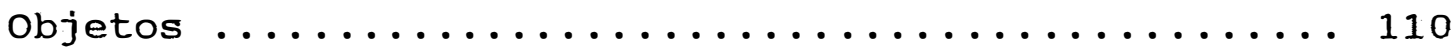

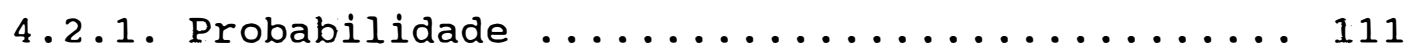

4.2.2. Avaliação de expressões ............... 118

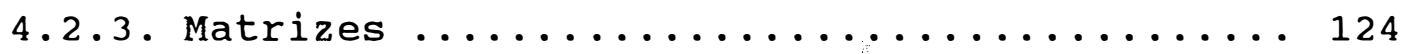

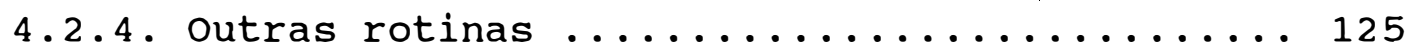

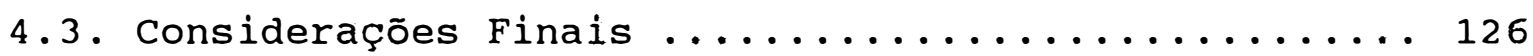

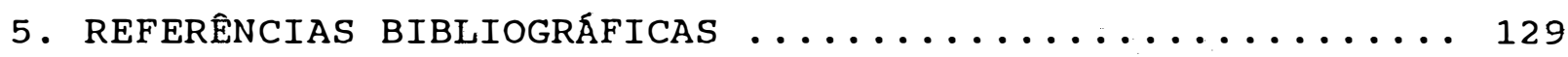

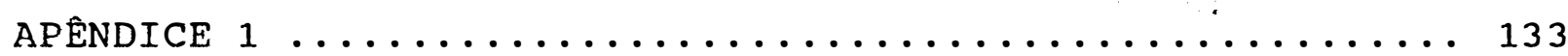

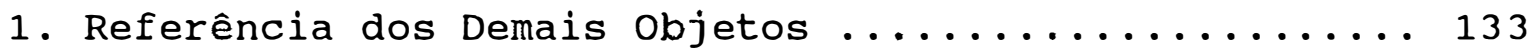

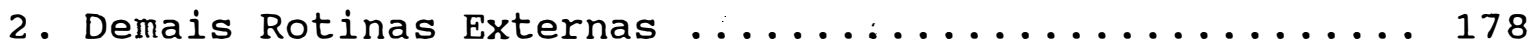

APENDICE $2 \ldots \ldots \ldots \ldots \ldots \ldots \ldots \ldots \ldots \ldots \ldots \ldots \ldots \ldots \ldots \ldots \ldots \ldots$ 


\section{LISTA DE TABELAS}

Tabela 1. Prioridade dos operadores na avaliação das expressões

Tabela 2. Operadores e respectivas prioridades na construção da expressão pós-fixa .................. 11

Tabela 3. Prefixos de algumas constantes do Turbo Vision ... 48

Tabela 4. Códigos de erro previstos por TDataFileMaker.Error 54

Tabela 5. Códigos de erros reconhecidos por TFilestruct .... 65

Tabela 6 . Possiveis valores do parâmetro op ........... 68

Tabela 7. Códigos de erro do objeto TMatrix ............ 89

Tabela 8. Modos de aplicação da transformação de

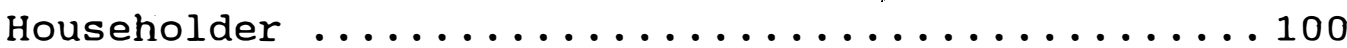

Tabela 9. Condições de falha no objeto TRandNum .......... 106

Tabela 10. Operadores aritméticos previstos em Éval .......118

Tabela 11. Operadores lógicos ou boleanos aceitos por Eval . 119

Tabela 12. Funções matemáticas disponíveis em Eval ........ 119

Tabela 13. Códigos de erro reconhecidos por Eval ..........120

Tabela 14. Condições de erro reconhecidos na especificação da expressão a ser avaliada ................122

Tabela 15. Eventos tratados por TBrowserDialog.HandleEvent . 134

Tabela 16. Códigos de erros reconhecidos por

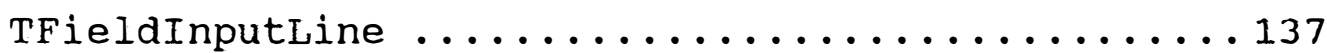

Tabela 17. Eventos tratados por TGenDataDlg.HandleEvent ....139

Tabela 18. Eventos tratados por TGenIfThenDlg. HandleEvent . 142

Tabela 19. Códigos de erros reconhecidos por THaltstream ... 144

Tabela 20. Eventos reconhecidos por TListDataDlg. HandleEvent ................. 148

Tabela 21. Eventos reconhecidos por

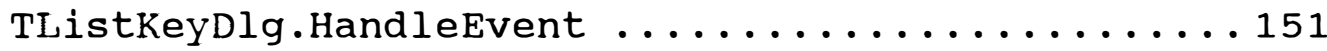

Tabela 22. Códigos de erro na especificação do critério de

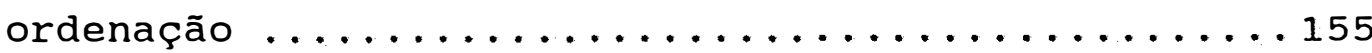


Tabela 23. Eventos reconhecidos por

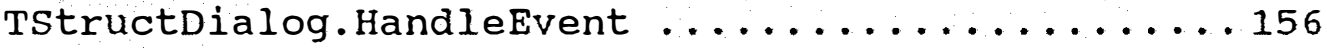

Tabela 24. Códigos de erros reconhecidos na obtenção dos

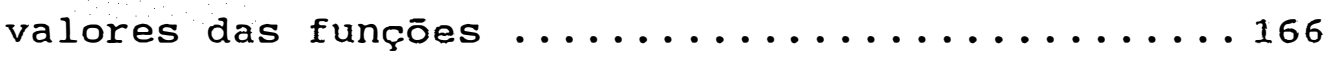




\section{LISTA DE FIGURAS}

Figura 1. Hierarquia dos objetos do Turbo Vision ........ 39 Figura 2. Árvore hierárquica após a inclusão de novos

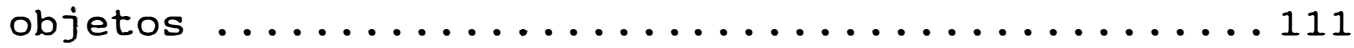




\title{
DESENVOLVIMENTO DE UMA BIBLIOTECA DE OBJETOS PARA USO EM PROGRAMAS DE MATEMÁTICA E DE ESTATÍSTICA
}

\author{
Autor: Amauri de Almeida Machado \\ Orientador: Prof. Dr. Cassio Roberto de Melo Godoi
}

\section{RESUMO}

A finalidade do presente trabalho foi construir uma biblioteca de objetos e outras rotinas que pudessem ser úteis para desenvolvimento de programas nas áreas de matemática e estatística. A linguagem utilizada foi Pascal, ambiente Turbo Pascal versão 6.0, e os programas foram todos escritos para utilização num equipamento IBM-PC ou compatível com sistema operacional MS-DOS ou compatível. A biblioteca de objetos foi toda desenvolvida como uma extensão da biblioteca Turbo Vision fornecida juntamente com a versão 6.0 do Turbo Pascal.

O desenvolvimento de uma biblioteca, tal como a que se propõe, deve-se a dois contextos muito interligados. O primeiro é o avanço da programação orientada a objetos (POO) como metodologia de programação e o segundo é a tendência atual de construção de bibliotecas para áreas específicas (frameworks). A POO se presta muito bem para a construção de bases para programas maiores, pois coloca um novo patamar aumentando a abstração e, consequentemente, a facilidade de desenvolvimento. As áreas específicas que se busca atingir neste caso são as de matemática e estatística. Assim, ela poderá ser muito útil como instrumento de apoio para programadores que atuam nessas áreas e como material didático em disciplinas de matemática numérica e estatística computacional.

Construiu-se um conjunto de objetos para tratar arquivos de dados estatísticos que serve também para exemplificar em detalhes a construção de uma aplicação Turbo Vision. Para tanto, foram desenvolvidos métodos para edição de dados, geração de novas colunas, acesso indexado e condicional ao disco, ordenação, seleção de chaves, acesso 
a dispositivos de entrada e saída, etc. Como toda aplicação Turbo Vision, é totalmente interativa e possui suporte para mouse, menus suspensos (pull-down), linha de status, cores, etc. Faz uso intensivo das ferramentas de interface fornecidas pelo Turbo Vision.

Foram desenvolvidos também objetos para a manipulação de matrizes e para a geração de variáveis aleatórias. O objeto para manipulação de matrizes sabe dimensionar-se e redimensionar-se seja quanto ao aumento de linhas ou de colunas, guardar-se e recuperar-se de um dispositivo de entrada e saída além de uma vasta gama de rotinas implementadas na forma de métodos cuja finalidade é dotar o objeto de um conjunto de ações que normalmente são efetuadas sobre matrizes. Outro objeto associado à matrizes estabelece uma lista ligada de matrizes sobre as quais poderão ser definidos procedimentos matriciais particulares ou gerais. Os objetos destinados à geração de variáveis aleatórias estão definidos para gerar variáveis uniformes segundo três metodologias diferentes e gerar variáveis de distribuição normal, gama, binomial e de Poisson. Um outro gerador de variáveis uniformes pode ser introduzido sem qualquer alteração na estrutura. Além disso, os objetos são facilmente extensíveis de modo que variáveis de outras distribuições possam ser geradas, aproveitando toda a estrutura já existente.

Todos os objetos poderão ser utilizados tal como se encontram, ou seja, todos eles são dotados de funcionalidade para muitas aplicações, embora a sua extensão seja possível e até desejável em muitos casos.

Também foi desenvolvido um conjunto completo de rotinas para a avaliação de funções, incluíndo funções de distribuição de probabilidade, avaliação de expressões matemáticas e tratamento de cadeias de caracteres. 


\title{
DEVELOPMENT OF AN OBJECT LIBRARY FOR USE IN MATHEMATICAL AND STATISTICAL PROGRAMS
}

\author{
Author: Amauri de Almeida Machado \\ Adviser: Prof. Dr. Cassio Roberto de Melo Godoi
}

\section{SUMMARY}

The objective of the current work was to build an object library and other routines that could be useful to the program development in the matematical and statistical areas. The programming language used was Pascal, on the Turbo Pascal version 6.0 environment, and the programs were all written to be used in an IBM-PC compatible equipment with MS-DOS compatible operational system. The object library was fully developed as an extension of the Turbo Vision library that comes together with Turbo Pascal version 6.0.

The development of a library as such as the one which is proposed is linked on two interconnected subjects. The first is the advance of Object Oriented Programming (OOP) as a metodology and the second is the present tendency of constructing libraries for specific areas (frameworks). The OOP fits well for constructing the bases for larger programms because establishes a new step increasing the abstraction and the facility of development. This library can be very useful as a support tool for programmers that work specifically in the mathematical and statistical areas and as a teaching material for courses in Numerical Analysis and Statistical Computing.

A set of objects was built to handle statistical data files, which by its turn has the objective of examplifying in details the construction of a Turbo Vision application. Furthermore, methods were developed for data edition, generation of new columns, input and output indexed access devices, etc. As a Turbo Vision application, it is totally interactive and has mouse support, pull-down menus, status line, colors, etc. It makes intensive use of the interface tools that come along with Turbo Vision. 
Objects were also developed to handle matrices and to generate random deviates. The object to handle matrices knows how to dinamically dimension and redimension itself with regards to the increase of lines and columns. It knows how to store and retrieve input and output devices, besides of a wide ammount of routines added in as methods with the purpose of providing to the object a set of actions that usually are performed on matrices. Another object associated to matrices establishes a linked list of matrices which allow the derivation of any particular or general matrix procedure. The objects to generate random deviates are defined for uniform deviates according to three different methods, and for normal, gamma, binomial and Poisson variables. Another generator of uniform deviates can be used without any change of the structure. Furthermore, the objects were built to allow easy extensions so that deviates with other distributions could be generated taking advantage of the structure already in.

All the objects can be used as they are. That is, all of them have the functionality for many applications. However, extensions of them will be possible and even desirable in many cases.

A complete set of routines was also developed for evaluation of functions, including probability distributions functions, evaluation of mathematical expressions and treatment of strings. 


\section{INTRODUÇÃO}

A eficiência no desenvolvimento de programas computacionais para todas as áreas e em particular para as áreas de estatística e matemática, está vinculada diretamente à disponibilidade de bibliotecas de apoio à programação. Este trabalho tem por objetivo propor uma concepção de desenvolvimento de uma biblioteca utilizando a técnica da programação orientada a objetos. A programação orientada a objetos é uma técnica avançada de produção de programas, sendo, devido às suas peculiares características, particularmente recomendada para a construção de bibliotecas.

Umá biblioteca de apoio à programação poderá ser útil para programadores experientes, permitindo uma melhor visualização e eficiência na produção de sistemas, para utilização com propósitos didáticos em disciplinas de matemática numérica ou estatística computacional e para usuários de computador que poderão eventualmente produzir algum programa para a resolução de um problema específico. A produção de bibliotecas científicas é uma atividade muito comum em outros países onde se nota que uma quantidade grande de pesquisadores que não são especialistas em programação ou em computadores produz os seus próprios programas sempre com auxílio de alguma biblioteca dentre as várias comercialmente distribuídas. Uma biblioteca com bons recursos permite a esse tipo de usuário uma dedicação quase que completa à resolução de seus problemas específicos, uma vez que não terá a necessidade de se preocupar com a escolha de algoritmos ou com detalhes específicos do equipamento. Esse enfoque permite que a programação possa ser desenvolvida através de "macros", de uma forma muito mais eficiente e genérica, uma vez que essas "macros" podem ser desenvolvidas na própria linguagem de programação sem a exigência de um grande período de aprendizado na linguagem e nas especificidades (algorítmicas, por exemplo) da aplicação, o que, talvez, seja o maior entrave para uma maior utilização dessa poderosa ferramenta.

A possibilidade de uma utilização mais ampla da programação de 
computadores como método auxiliar na solução de problemás cresce mais ainda com a extraordinária evolução da linguagem Pascal e do ambiente de programação desenvolvido pela BORLAND INTERNATIONAL. O Pascal é sabidamente uma linguagem de aprendizado relativamente fácil, é bastante formal e estruturada o que, em geral, conduz a bons hábitos de programação. Pelas suas qualidades, possui ampla penetração nas camadas onde são desenvolvidas aplicações científicas. A biblioteca, por estar vinculada ao TURBO PASCAL, está, em princípio, vinculada à equipamento compatível com IBM-PC e ao sistema operacional MSDOS ou compatível.

A programação orientada a objetos é uma técnica de desenvolvimento de programas relativamente recente. Entretanto, segundo a quase unanimidade dos especialistas na área, será a técnica majoritária e amplamente utilizada num futuro muito próximo. Alguns afirmam que ela representará nos anos 90 o que a programação estruturada representou nos anos 70 e 80. As suas características de encapsulamento, herdabilidade e polimorfismo permitem que nada já existente seja reinventado e sim herdado, estendido e apenas em alguns aspectos (já previstos) modificado. O ambiente TURBO PASCAL já suporta a programação orientada a objetos desde a versão 5.5 , mas foi com a introdução da biblioteca TURBO VISION, a partir da versão 6.0 , que a técnica foi realmente colocada numa forma que possibilita o desenvolvimento de aplicações sem um investimento exagerado em treinamento. O TURBO VISION, dentre outras facilidades, colocou à disposição dos programadores uma complexa hierarquia de objetos que praticamente elimina, por exemplo, um conhecimento mais profundo dos detalhes do equipamento e do sistema operacional. Ou seja, mudou o nível do desenvolvimento.

O presente trabalho tem por objetivo colocar um novo patamar nesse nível. Através das propriedades da programação orientada a objetos, é possível aproveitar todas as características da biblioteca TURBO VISIONe extendê-la para um direcionamento de interesse, onde se procurará fornecer ferramentas para resolução de problemas na área de matemática e estatística. Assim, a biblioteca ora apresentada, no que se refere aos seus objetos, não é uma nova biblioteca e sim uma extensão do TURBO VISION.

A biblioteca proposta inclui objetos para tratamento de arquivos, para álgebra de matrizes, para a geração de variáveis aleatórias e rotinas para a avaliação de funções 
e integrais de algumas funções de interesse particular da estatística como, por exemplo, as integrais que definem a função gama e as envolvidas no cálculo de probabilidades, relativas às distribuições mais utilizadas. Os objetos para tratamento de arquivos foram desenvolvidos com a finalidade de propor uma estrutura de arquivos adequada para o armazenamento de dados estatísticos e para exemplificar a construção de uma aplicação interativa TURBO VISION, utilizando a maioria dos seus objetos de interface. Procura-se mostrar que, através desses objetos, é possível construir aplicações avançadas, de grande compatibilidade em termos de interface com outros programas, tornando o aprendizado muito mais simples e rápido. Além disso, procura-se mostrar, também, que é possível criar uma estrutura de arquivos de dados estatísticos de manuseio relativamente sofisticado de forma totalmente interativa sem a necessidade de uma única linḩa de programa.

A documentação da biblioteca, apresentada na seção de resultados, segue de perto a documentação utilizada no TURBO VISION USER'S GUIDE. Basicamente, descreve o modo como os métodos e os campos dos objetos poderão ser utilizados e indica as finalidades de cada um. Também são descritas todas as variáveis, tipos e constantes utilizadas, além das rotinas que não compõem a hierarquia de objetos de uma forma semelhante à descrição dos objetos.

Note-se que não existe em todo o trabalho nenhum resultado de teste de eficiência, velocidade ou qualquer coisa do gênero. Procurou-se, entretanto, utilizar algoritmos e implementações já consagrados na literatura referente a cada assunto específico e, sempre que possível, colocando alternativas quando uma rotina pudesse falhar em algum ponto importante. Alguns deles poderão não ser os melhores disponíveis, como o ShellSort, por exemplo, mas são os que melhor se adaptaram aos objetivos propostos.

Finalmente, seria interessante tecer alguns comentários acerca da terminologia utilizada em algumas situações. Uma dificuldade clássica na área é encontrar termos em português correspondentes aos respectivos na língua inglesa. O termo string, por exemplo, usualmente traduzido como cadeia (indicando uma cadeia ou uma sequência de caracteres alfanuméricos), embora isso possa trazer dificuldades de compreensão. Outros são de tradução mais difícil ainda (senão impossível), como os termos stream e view. Um stream é, na realidade, uma seqüência de bytes que poderá ser enviada a algum dispositivo. 
Tipicamente, poderá ser um arquivo em disco, mas o conceito é mais amplo que arquivo já que esse dispositivo poderá ser, por exemplo, a memória expandida ou uma porta de comunicação. Uma view é um objeto de interface que caracteristicamente mostra alguma coisa na tela, mas é, também, um conceito muito mais amplo do que isso. A aplicação inteira, por exemplo, é conceitualmente uma view. Outros termos de grande importância na programação orientada a objetos são early binding e late binding que indicam como é feita a atribuição do endereço de um procedimento a ser chamado num determinado momento, se durante a compilação ou depois (durante a execução). 


\section{REVISÃO DE LITERATURA}

\subsection{Programação Orientada a Objetos (POO) e o Pascal}

O texto que segue é baseado na excelente documentação do Turbo Pascal versão 6.0, mais especificamente no capítulo 4 do USER's GUIDE, que é a referência recomendada para uma descrição mais detalhada das características e capacidades da POO. A programação orientada a objetos também é muito bem descrita em EZZEL (1989) enquanto que uma descrição mais resumida da POO pode ser encontrada em O'BRIEN (1991).

Como o próprio nome diz, a programação orientada a objetos está relacionada com o conceito de objeto, ou seja, algo que possui características e comportamento ou funcionalidade. Todos os objetos são assim. Uma cadeira, um automóvel, um avião, um televisor, etc. Um automóvel, por exemplo, possui muitas características (carroceria de metal, tipo e potência do motor, tipo e largura de pneus, cor, etc.) e uma funcionalidade básica que é servir como meio de transporte ou de locomoção. Note-se que um objeto que possuir uma funcionalidade completa (um televisor ou um carro, por exemplo) pode ser utilizado da forma como foi produzido e toda a complexidade interna desse objeto, em princípio, não interessa ao usuário comum, ou pelo menos não há necessidade do seu conhecimento para a utilização do objeto.

Outros objetos possuem características e funcionalidade próprias, mas que são insuficientes para o objetivo para o qual se deseja destiná-los. Pode-se, então, aproveitar essas características já existentes e derivar a partir deles outros objetos que além das características já existentes no anterior, possuam outras mais especializadas. Está-se, dessa forma, adicionando novas características e nova funcionalidade àquelas já existentes.

A POO toma vantagem de todas essas características dos objetos. Em 
termos de programação, um objeto pode ser definido como a junção numa única entidade de dados (que definem as suas características) e programas (que definem a sua funcionalidade), ou resumidamente, Código e Dados = Objeto. Essa definição possui três propriedades básicas e fundamentais: encapsulamento, hereditariedade e polimorfismo, que traduzem toda a sua filosofia, descritas nos sub-ítens de 2.1.1. a 2.1.3.

\subsubsection{Encapsulamento}

É a combinação de dados e programas numa única entidade. É a propriedade que permite que os objetos sejam considerados como entidades autônomas, dotados de características e de funcionalidade. O encapsulamento poderia ser definido através da seguinte condição: o acesso aos dados (chamados de campos) de um objeto somente poderá ser feito através de rotinas (chamadas de métodos) do próprio objeto. O benefício primário do encapsulamento é que, sendo o acesso aos campos limitado apenas aos métodos do objeto, existe uma maior liberdade e segurança nas alterações que necessitem ser feitas nos campos do objeto, evitando efeitos colaterais.

\subsubsection{Hereditariedade}

A hereditariedade existente numa hierarquia de objetos funciona exatamente como aquela utilizada na taxonomia de animais ou vegetais. Assim, os animais, por exemplo, podem se dividir em vertebrados e invertebrados. Dentro da divisão dos vertebrados existem mais divisões, mas as características que possuem os vertebrados são comuns a todas as divisões que se sucedem. Note-se, também, que essas características são repassadas por herança aos descendentes e quanto mais se aproxima do final da hierarquia mais especializadas se tornam as subdivisões.

A hereditariedade, tal como utilizada pela POO funciona de maneira parecida. Os objetos que estão acima na hierarquia (os ancestrais) repassam suas características (os campos) e sua funcionalidade (seus métodos) a todos os seus descendentes. A hereditarieda- 
de é, portanto, o princípio chave da reutilização de código e dados já definidos. Em geral, os objetos do topo da hierarquia são objetos genéricos e o princípio da funcionalidade crescente ou da especialização se torna cada vez mais evidente quando se desce na hierarquia.

\subsubsection{Polimorfismo}

O termo polimorfismo tem sua origem na língua grega e quer dizer "de muitas formas". Na POO é um meio de se dar um nome a uma ação que é compartilhada acima e abaixo da hierarquia de objetos, sendo que cada um implementa a ação segundo as suas próprias especificidades, muitas vezes aproveitando, também, as ações já definidas pelos seus ancestrais.

O polimorfismoé, portanto, um dos pilares da extensibilidade da hierarquia de objetos. A idéia fundamental dentro desse processo são os métodos virtuais. Os métodos virtuais implementam um tipo de ação que é definido somente em tempo de execução. Eles se contrapõem aos métodos estáticos, cuja chamada deve ser rigorosamente definida em tempo de compilação. Assim, durante a compilação existe a denominada "ligação anterior" (early binding), apropriada aos métodos estáticos, onde o compilador define exatamente a ação a ser tomada numa determinada circunstância. Em algumas situações, entretanto, não é desejável definir com antecedência qual ação será tomada, uma vez que ela dependerá do encaminhamento da execução do programa. Isto exigirá do compilador um tipo de ligação denominada "ligação posterior" (late binding). Os métodos virtuais, juntamente com a definição de objetos como entidades de alocação dinâmica, formam o conjunto de condições fundamentais para a criação de objetos polimórficos, de modo que a extensibilidade de uma hierarquia de objetos está estreitamente relacionada ao uso de métodos virtuais.

Como se pode ver, a filosofia de implementação através da POO é muito distinta da programação tradicional. Através do exposto até agora pode-se ter uma idéia das imensas potencialidades dessa filosofia de organização. Se as características de encapsulamento, herdabilidade e polimorfismo forem utilizadas adequadamente, ter-se-á um código mais estruturado, mais modular e abstrato (no sentido de esconder os detalhes) e reutilizável. Tudo isto adiciona ao código maior estruturação, extensibilidade e facilidade de manutenção. 


\subsection{Alguns Algoritmos Básicos}

\subsubsection{Avaliação de expressões}

O texto que segue é inteiramente baseado em HOROWITZ \& SAHNI (1984), onde poderão ser encontrados maiores detalhes acerca da metodologia de avaliação de expressões e da forma de descrição algorítmica utilizada pelos autores, o SPARKS.

A dificuldade na avaliação de expressões pode ser colocada segundo dois aspectos básicos: como interpretar e como resolver expressões aritméticas complexas tais como:

$$
\mathrm{X}=\mathrm{A} / \mathrm{B}^{\wedge} \mathrm{C}+\mathrm{D}^{*} \mathrm{E}-\mathrm{A}^{*} \mathrm{C}
$$

ou

$$
\mathbf{Y}=\mathbf{A} *(\mathbf{B}+\mathbf{C}) * \mathbf{D}
$$

onde * representa a multiplicação e ^ a exponenciação.

Segundo os autores, a solução que se tem hoje é tão simples quanto elegante, o que tornou a construção de compiladores uma tarefa elementar dentro da ciência da computação.

Uma expressão é formada de operandos, operadores e delimitadores. Os operandos das expressões anteriores são todos caracteres, por facilidade de representação. Poderão ser quaisquer seqüências de caracteres com um determinado significado (Tokens), incluindo nomes de variáveis ou constantes. Os valores que as variáveis podem assumir devem ser consistentes com as operações que podem ser feitas com elas.

Para fixar a ordem de avaliação de uma expressão, atribui-se uma prioridade a cada operador. Entende-se, a partir daí, que dentro de qualquer par de operadores serão avaliados sempre os operadores de mais alta prioridade.

Os tipos mais comuns são os operadores aritméticos, os boleanos e os relacionais. Um conjunto de operadores com a sua prioridade usual pode ser visto na Tabela 1. 
Tabela 1. Prioridade dos operadores na avaliação das expressões

\begin{tabular}{ll} 
Operadores & Prioridade \\
\hline , - unário & 6 \\
$*$, , & 5 \\
,+- & 4 \\
$<,>, \leq, \geq,=, \neq$ & 3 \\
and (e) & 2 \\
or (ou) & 1 \\
\hline
\end{tabular}

Os operadores de mesma prioridade são avaliados da esquerda para a direita, como, por exemplo, os relacionais e os da soma algébrica. Uma vez estabelecidos os operadores e as respectivas prioridades, pode-se decidir como a expressão será avaliada. A maneira mais simples e elegante é a sua re-expressão numa outra forma denominada "pós-fixa" (contrariamente à forma original, denominada "infixa", por possuir os operadores entre os operandos) ou notação polonesa reversa. Uma forma pós-fixa possui muitas vantagens em relação à sua correspondente infixa. Primeiro, não há mais necessidade de parênteses e, segundo, deixa de existir prioridade entre os operadores. Evidentemente, tudo isso é levado em consideração pelo algoritmo de construção da expressão pós-fixa. A expressão pode ser avaliada fazendo o exame da esquerda para a direita, empilhando os operandos e analisando os operadores. Usa-se os operandos como números da pilha e, finalmente, coloca-se o resultado na pilha.

HOROWITZ \& SAHNI (1984) estabelecem o algoritmo seguinte, para avaliação de expressões pós-fixadas:

\section{procedure EVAL(E)}

" $\quad$ //Avalia expressão E. Assume-se que o último caráter em E é um ';' (ponto e vírgula). O procedimento NextToken está sendo utilizado para extrair o último ítem de E. O ítem pode ser um operando, um operador ou um ';'. Um arranjo unidimensional Stack(1:n) está sendo utilizado como uma pilha. 


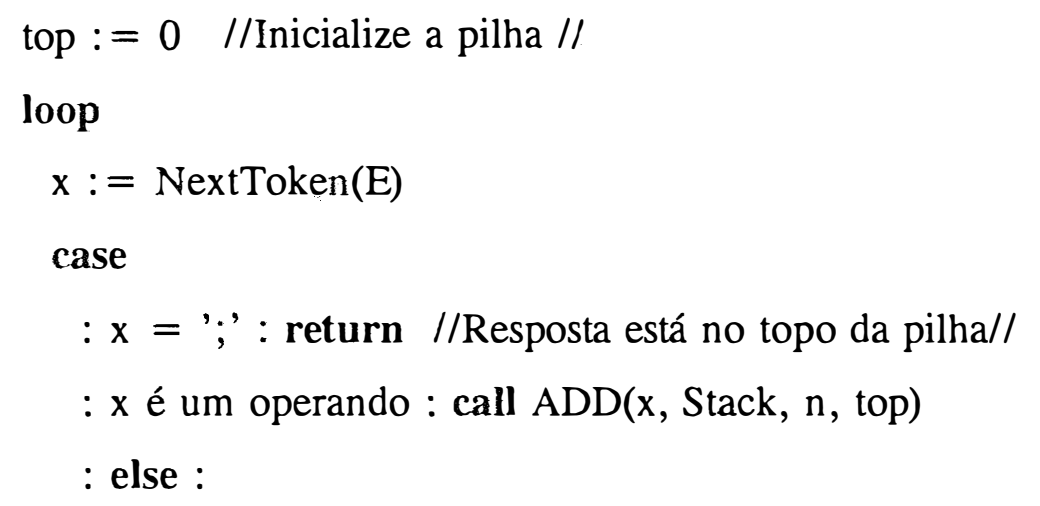

Retire a quantidade certa de operandos para operador $\mathrm{x}$ de Stack, execute a operação e armazene o resultado, se hou- ver, dentro da pilha.

end

forever

end EVAL

onde os procedimentos para a colocação e a retirada de elementos na pilha são dados por:

procedure $\mathrm{ADD}$ (item, Stack, $\mathrm{n}$, top)

//Insere um item em Stack de tamanho máximo n. Top é a quantidade de itens que atualmente se encontra em Stack//

if top $\geq \mathrm{n}$ then call Stack_Full

top $:=$ top +1

Stack(top) $:=$ item

end ADD

procedure DELETE(item, Stack, top)

//Remove um elemento do topo de Stack, se Stack não está vazia, e o armazena em item//

if top $\leq 0$ then call Stack_Empty

item $:=$ Stack(top)

top $:=$ top -1

end DELETE 
Na construção da expressão pós-fixa existe uma prioridade de chegada dos operadores dentro da pilha e uma prioridade dos operadores dentro da pilha, resumidas na Tabela 2.

Tabela 2. Operadores e respectivas prioridades na construção da expressão pós-fixa

\begin{tabular}{lll}
$\begin{array}{c}\text { Símbolo } \\
(\mathrm{x})\end{array}$ & $\begin{array}{l}\text { Prioridade dentro } \\
\text { da pilha ISP }(\mathrm{x})\end{array}$ & $\begin{array}{l}\text { Prioridade de } \\
\text { chegada ICP(x) }\end{array}$ \\
\hline \multirow{2}{*}{, / } & - & - \\
,+- & 3 & 4 \\
( & 2 & 2 \\
\hline
\end{tabular}

A regra será: os operadores serão retirados da pilha até que sua prioridade dentro da pilha "isp" permaneça maior ou igual a prioridade entrante "icp" do novo operador. $\operatorname{ISP}(\mathrm{x})$ e $\operatorname{ICP}(\mathrm{x})$ são funções que refletem a lógica da Tabela 2.

\section{procedure PostFix $(\mathrm{E})$}

//Converte a expressão infixa $\mathrm{E}$ em pós-fixa. O último caráter de $\mathrm{E}$ é um ';', que também será o último caráter da pós-fixada. O procedimento NextToken devolve o próximo ítem (operador, operando ou delimitador, o que chegar primeiro). $\operatorname{Stack}(1: n)$ está sendo utilizado como uma pilha e o caráter " com ISP(") = -1 é usado como indicador de fundo da pilha. ISP e ICP são funções//

Stack(1):=" : top $:=1$ //Inicializa a pilha//

Joop

$$
\mathrm{x}:=\operatorname{NextToken}(\mathrm{E})
$$

case

$: \mathrm{x}=$ ';' : while top $>1$ do //Esvazie a pilha// 


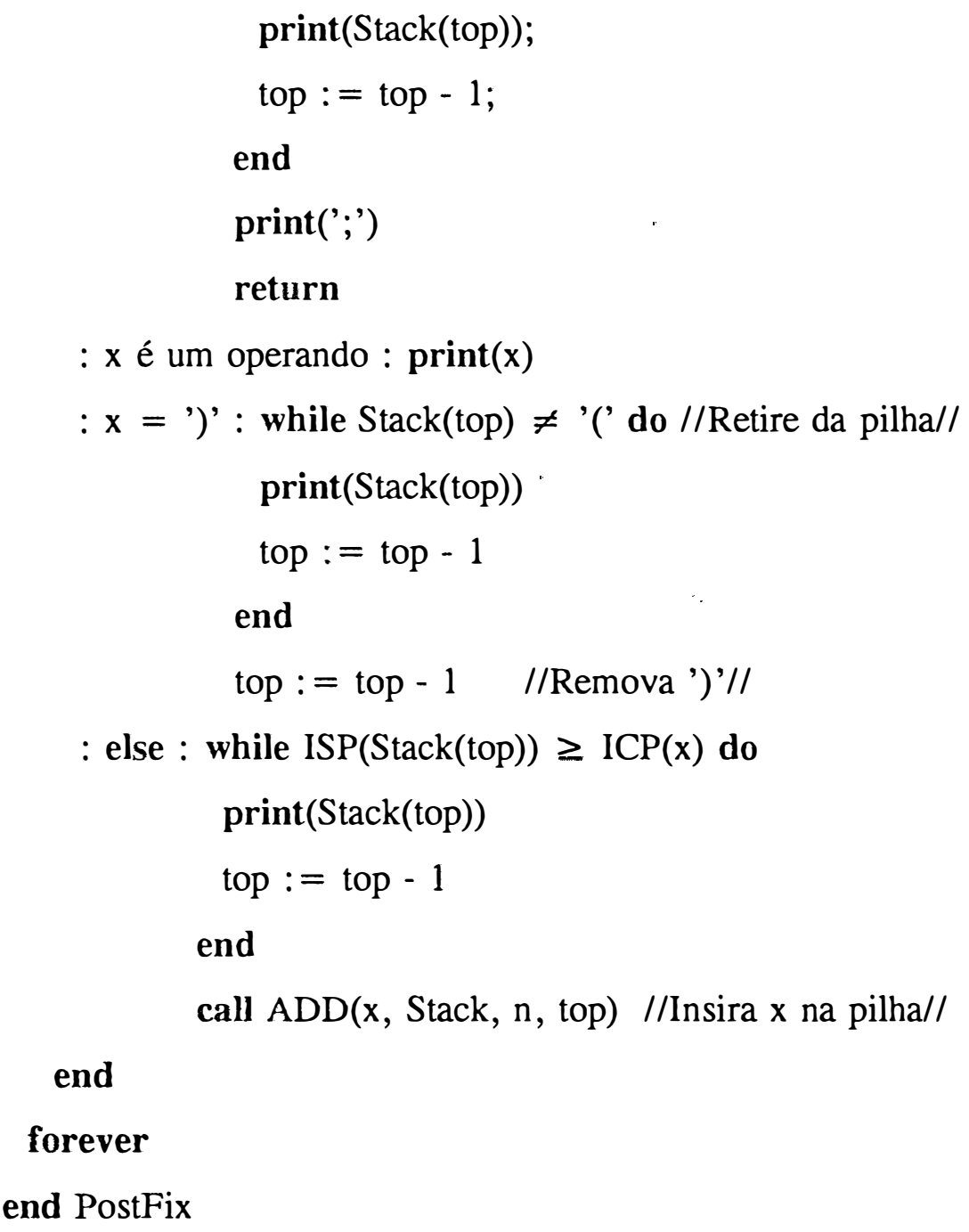

Quanto ao tempo de computação, o algoritmo faz apenas uma passagem através da entrada. Se a expressão tem n símbolos (ítens), então a quantidade de operações será alguma constante vezes $\mathrm{n}$. A pilha não será mais profunda do que a quantidade de operadores mais 1.

\subsubsection{Ordenação}

COOK et alli (1985) descrevem o algoritmo ShellSort, através de uma ilustração, da seguinte maneira:

Considere-se que se deseja ordenar $n=12$ valores $7,9,2,11,4,1,3$, 
$10,5,6,2,8$, em ordem crescente.

$1^{\circ}$ passo: Inicialmente divide-se o conjunto em dois grupos de tamanho Gap através da operação Gap $=$ Gap div 2, sendo n o valor inicial de Gap. Inicia-se pela comparação de todos os pares de valores decorrentes da subdivisão. Ordena-se, agora, todos os 6 grupos de 2 componentes cada (um * indica onde as trocas serão feitas):

$\begin{array}{lc}7 & 3 * \\ 9 & 10 \\ 2 & 5 \\ 11 & 6 * \\ 4 & 2 * \\ 1 & 8\end{array}$

Efetuando as trocas e intercalando os grupos, o conjunto fica:

$$
3,9,2,6,2,1,7,10,5,11,4,8
$$

$2^{\circ}$ passo: Gap agora vale 3 (Gap = Gap div 2), ou seja, existem 4 grupos de 3 componentes cada:

$$
\begin{array}{cccc}
3 & 6 & 7 & 11 \\
9 & 2 & 10 & 4 * \\
2 & 1 & 5 & 8^{*}
\end{array}
$$

Como se pode ver, somente nas linhas 2 e 3 são necessárias as trocas, de modo que cada uma fique na ordem desejada, ou seja:

$$
\begin{array}{rrrr}
3 & 6 & 7 & 11 \\
2 & 4 & 9 & 10 \\
1 & 2 & 5 & 8
\end{array}
$$

A seguir, os grupos são novamente intercalados e o conjunto fica:

$$
\begin{array}{lllllllllllll}
3 & 2 & 1 & 6 & 4 & 2 & 7 & 9 & 5 & 11 & 10 & 8
\end{array}
$$

$3^{\circ}$ Passo: Para Gap = Gap div 2 tem-se Gap igual a 1, ou seja, trabalhar-se-á com 
12 grupos de tamanho 1 ou todo o conjunto, de onde vem a ordenação final. Assim, fica, finalmente:

\section{$\begin{array}{lllllllllllll}1 & 2 & 2 & 3 & 4 & 5 & 6 & 7 & 8 & 9 & 10 & 11\end{array}$}

$4^{\circ}$ Passo: Gap $=$ Gap div $2=0$ encerra o processo.

No método ShellSort pode-se perceber duas características importantes: primeira, é um algoritmo de ordenação "no lugar", ou seja, não exige nenhum armazenamento intermediário para fazer a ordenação e, segunda, é um procedimento do tipo "dividir para conquistar", ou seja, a ordenação não é feita (a não ser no final quando muito da ordenação já foi feita) comparando-se todos os elementos e sim grupos deles, cada vez maiores. COOK et alli (1985) apresentam alguns dados referentes à comparação desse algoritmo com o QuickSort, o TreeSort e outros. Não é mais rápido, por exemplo, que o Quicksort, mas pode ser considerado um processo de boa qualidade, mesmo para conjuntos muito grandes, muito superior a alguns algoritmos, como o BubbleSort e o ExchangeSort.

Como se pode notar, o algoritmo de ordenação discutido é capaz de ordenar somente conjuntos unidimensionais. O'BRIEN (1988) apresenta, dentro do contexto do TurboSort, que é uma rotina de ordenação do Turbo Pascal Database Toolbox, um algoritmo capaz de generalizar o processo de ordenação para um número qualquer de campos de forma hierárquica, estabelecendo, ainda, que tipo de ordenação se deseja para cada campo, ascendente ou descendente. Também os campos podem armazenar qualquer tipo de variável: real, string, integer, etc. O algoritmo completo, programado para uso com o TurboSort, é fornecido pelo mesmo autor.

\subsubsection{Geração de índices.}

Freqüentemente, colunas de arquivos precisam ser referenciadas através de um conjunto de índices expressos por númerosinteiros e muitas vezes esses índices possuem uma regra de formação. BAKER (1986) apresenta uma maneira extremamente simples e rápida de gerar blocos de valores que correspondem a esses índices que possuem uma regra de 
formação. Assim, o i-ésimo valor de um vetor de inteiros será dado por:

$$
\operatorname{Mod}([(i-1) / y], x)+1
$$

onde Mod representa o operador módulo, e $]$ representa a parte inteira. Fazendo i variar de 1 até $n$, por exemplo, essa função gera blocos de valores inteiros onde aos primeiros y valores é dado o valor 1 , aos y seguintes o valor 2 e assim por diante. Este processo continua até que $\mathrm{y}$ valores $\mathrm{x}$ sejam atribuídos aos componentes do vetor. Se $\mathrm{n}$ ainda não foi atingido, o processo se repete até que i seja igual a $n$.

\subsubsection{O método de Gauss-Jordan}

O método de Gauss-Jordan é um dos mais antigos métodos de solução de sistemas de equações, ainda hoje muito utilizado. Além de ser um processo simples e fácil de entender, muitos métodos que possuem um comportamento superior são, na realidade, adaptações maiores ou menores desse método (como, por exemplo, o SWEEP e os métodos de decomposição).

Seguindo o modo como é apresentada por PRESS et alli (1989), a eliminação de Gauss-Jordan considera o problema expresso em (1):

$$
A \cdot[X \mid Y]=[B \mid I]
$$

onde $\mathbf{A}$ é a matriz $\mathrm{n} \times \mathrm{n}$ dos coeficientes do sistema, $\mathbf{X}$ é a matriz $\mathrm{n} \times \mathrm{p}$ que contém os vetores das incógnitas, $\mathbf{B}$ é a matriz $\mathrm{n} \times \mathrm{p}$ dos vetores do segundo membro e $\mathbf{I}$ é a matriz identidade $\mathrm{n} \times \mathrm{n}$. O método se propõe a resolver, simultaneamente, o conjunto de sistemas lineares (2):

$$
A \cdot x_{1} \quad A \cdot x_{2} \quad \ldots \quad A \cdot x_{p}
$$

implicando que:

$$
A \cdot Y=I
$$

ou seja, que a inversa $\mathbf{Y}$ de A é um subproduto do processo de solução. 
O método de Gauss-Jordan tem por base o fato de que operações elementares (trocas de linhas, de colunas e substituição de uma linha por uma combinação linear dela própria com uma outra) realizadas sobre $\mathbf{A}$ não alteram nem a solução $\mathbf{X}$ nem a matriz $\mathbf{Y}$, desde que operações idênticas também sejam realizadas sobre essas últimas matrizes. Evidentemente, toda troca que for feita deverá ser desfeita ao final para que as soluções correspondam à ordem inicial das incógnitas.

Uma vez que são possíveis trocas de linhas e colunas dentro do processo de Gauss-Jordan, o processo recebe o nome de Gauss-Jordan sem pivoteamento, com pivoteamento parcial e com pivoteamento total se, respectivamente, não for feita nenhuma troca de linhas e de colunas, se forem feitas somente trocas de linhas e se forem feitas trocas de linhas e de colunas. Este processo recebe o nome de pivoteamento porque um elemento vital dentro do processo é o pivô, elemento da diagonal que divide todos os elementos da linha respectiva. Segundo PRESS et alli (1989), não é óbvio, porém é verdadeiro, que a aplicação da eliminação de Gauss-Jordan sem pivoteamento traz uma instabilidade numérica muito grande, mesmo quando não existe um pivô igual a zero. Por esse motivo, a eliminação de Gauss-Jordan não deve ser utilizada sem pivoteamento. O critério para as trocas de linhas (e/ou de colunas) passa a ser, então, colocar como elemento da diagonal o maior valor disponível. Esse é um bom critério, embora não esteja protegido para uma eventual mudança de escala numa determinada equação.

A eliminação de Gauss-Jordan possui dois problemas básicos. Primeiro, a matriz dos coeficientes sempre precisará ser invertida, mesmo que a inversão não seja necessária e, segundo, todos os vetores do segundo membro precisam estar disponíveis no mesmo momento na memória.

\subsubsection{A decomposição $\mathrm{LU}$ e relacionados}

A decomposição LU recebe esse nome porque consiste na fatoração de uma matriz quadrada numa matriz triangular inferior e numa triangular superior, ou seja:

$$
L \cdot U=A
$$


onde $\mathbf{L}$ é a matriz triangular inferior e $\mathbf{U}$ é a triangular superior. Assim sendo, o sistema de equações pode ser reescrito como

$$
A \cdot x=(L \cdot U) \cdot x=L \cdot(U \cdot x)=b
$$

que pode ser resolvido inicialmente para o vetor $\mathbf{y}$ tal que:

$$
L \cdot y=b
$$

e, depois, resolvendo

$$
U \cdot x=y
$$

A grande vantagem dessa decomposição é que a solução de um sistema é quebrada para a solução de dois outros sistemas de solução muito simples, pois são triangulares. A solução do primeiro sistema é chamada de solução "para frente" uma vez que resolve a primeira equação, depois a segunda e assim por diante. No segundo sistema, como a matriz é triangular superior, o sistema é resolvido "para trás". Assim, por exemplo, a solução de (7) é obtida através de (8).

$$
\begin{aligned}
& x_{n}=\frac{y_{n}}{u_{n n}} \\
& x_{1}=\frac{1}{u_{11}}\left[y_{1}-\sum_{j=1+1}^{n} u_{i f} x_{f}\right] \quad i=n-1, n-2, \ldots, 1
\end{aligned}
$$

Como se pode notar, com a decomposição $\mathrm{LU}$, a matriz dos coeficientes não precisa ser invertida, se isso não for necessário, e o processo pode ser aplicado independentemente a cada vetor $\mathbf{b}$ do segundo membro.

PRESS et alli (1989) descrevem com detalhes o modo como é feita a decomposição que, resumidamente, consiste no seguinte:

No conjunto de equações, retiradas de $\mathbf{L U}=\mathbf{A}$, como colocado em (9), pode-se obter uma solução para os l's e u's, estabelecendo, inicialmente, a restrição $l_{\mathrm{ii}}=1$, através do algoritmo de Crout, listado na próxima página. 


$$
\begin{array}{ll}
i<j: & I_{i 1} u_{1 j}+I_{i 2} u_{2 j}+\ldots+I_{i j} u_{i j}=a_{i j} \\
i=j: & I_{i 1} u_{1 j}+I_{i 2} u_{2 j}+\ldots+I_{i j} u_{j j}=a_{i j} \\
i>j: & I_{i 1} u_{1 j}+I_{i 2} u_{2 j}+\ldots+I_{i j} u_{j j}=a_{i j}
\end{array}
$$

Para $\mathrm{i}=1, \ldots, \mathrm{n}$ faça $I_{i i}=1$

Para $\mathrm{j}=1,2, \ldots, \mathrm{n}$ faça .

Para $\mathrm{i}=1,2, \ldots, \mathrm{j}$, utilize a restrição e (9) para encontrar $I_{i j}=a_{i j}-\sum_{k=1}^{i-1} I_{i k} u_{k j}$ Para $\mathrm{i}=\mathrm{j}+1, \mathrm{j}+2, \ldots, \mathrm{n}$ utilize (9) e resolva para l através de $I_{i j}=\frac{1}{u_{j j}}\left(a_{i j}-\sum_{k=1}^{j-1} I_{i k} u_{k j}\right)$

No algoritmo de Crout, cada $\mathrm{a}_{\mathrm{ij}}$ é utilizado apenas uma vez, de onde se conclui que, à exceção dos $l_{\text {ii }}$ que são iguais a 1, todos os demais valores da decomposição podem ser colocados no local onde está a matriz A, ou seja, a decomposição é feita "no lugar" e nenhum outro local de armazenamento é necessário. Assim, para uma matriz $4 \times 4$, o algoritmo de Crout transforma, no seu estágio final, a matriz original na forma (10).

$$
\left[\begin{array}{llll}
u_{11} & u_{12} & u_{13} & u_{14} \\
I_{21} & u_{22} & u_{23} & u_{24} \\
I_{31} & I_{32} & u_{33} & u_{34} \\
I_{41} & I_{42} & I_{43} & u_{44}
\end{array}\right]
$$

Segundo PRESS et alli (1989), apenas o pivoteamento parcial é possível de ser feito de forma eficiente dentro do algoritmo, mas que ele é suficiente para dar estabilidade numérica ao método. Assim, na realidade, não se faz a decomposição da matriz $\mathbf{A}$ :e sim de uma matriz resultante da permutação de linhas de A. Isto não traz nenhuma complicação se, de alguma maneira, for armazenada a permutação feita nas linhas. Os mesmos autores trazem uma explicação detalhada do modo como é feito o pivoteamento e o método é chamado de método de Crout com pivoteamento implícito parcial. 
Além da solução do sistema de equações, existem outros aspectos importantes relacionados à decomposição LU. O primeiro deles é a inversão da matriz A. A inversão da matriz A é feita (e, segundo os autores, não há melhor maneira de se fazer isto) coluna a coluna, como se estivesse resolvendo um sistema de equações onde os $n$ vetores do segundo membro são unitários. Este processo tem como inconveniente básico o fato de que a inversão não é feita no local, ou seja, a matriz original é perdida (na decomposição) mas não pode ser substituída pela inversa e, além disso, ainda um outro vetor, que irá armazenar o vetor unitário, precisa ser dimensionado. O segundo aspecto é o cálculo do determinante da matriz A. Como $\mathbf{U}$ é triangular superior e está completa, o determinante de A será simplesmente o produto dos elementos da diagonal de $\mathbf{U}$, levando-se em conta a permutação das linhas de $\mathbf{A}$.

\subsubsection{O método de Cholesky e relacionados}

O método de Cholesky é usualmente utilizado para a solução do sistema de equações normais, onde a matriz dos coeficientes é simétrica e positiva semidefinida. É estreitamente relacionado à decomposição LU. Entretanto, por fazer uso dessas características, a eliminação de Cholesky possui excelentes propriedades de economia de memória e estabilidade numérica. Dentro do contexto de mínimos quadrados, tem-se o sistema:

$$
A \cdot b=d
$$

onde $\mathbf{A}=\mathbf{X}^{\mathrm{T}} \mathbf{X}$ e $\mathbf{d}=\mathbf{X}^{\mathrm{T}} \mathbf{y}$.

Assim, como visto na decomposição LU, a matriz A sempre poderá ser fatorada. Desse modo, o método de Cholesky se baseia no fato de que sempre existe uma matriz triangular superior (real) $\mathbf{U}$ tal que (12) seja satisfeita. A solução b poderá, assim como na decomposição LU, ser obtida através dos sistemas expressos em (13) e (14).

$$
\begin{aligned}
& U^{T} U=A \\
& U^{T_{y}}=d
\end{aligned}
$$

O processo inicial de obtenção da matriz $\mathbf{U}$ é muito parecido com o da 


$$
U \cdot b=y
$$

obtenção da LU. Ela pode ser facilmente construída ocupando os lugares correspondentes na matriz simétrica $\mathbf{A}$, segundo o algoritmo que segue:

$$
\begin{aligned}
& \text { Passo 1. Para } i=1 \text { até n faça } a_{i j}=\left(a_{i j}-\sum_{k=1}^{i-1} a_{k i}^{2}\right)^{1 / 2} \\
& \text { Passo 2. Para } j=i+1 \text { até n faça } a_{i j}=\frac{\left(a_{i j}-\sum_{k=1}^{i-1} a_{k i} a_{k j}\right)}{a_{i j}}
\end{aligned}
$$

A matriz A é teoricamente positiva semidefinida, mas erros de arredondamento poderão ocorrer e tornar o radical do primeiro passo negativo. Isto pode ser previsto na implementação, anulando-se toda a linha de $\mathbf{U}$ onde o valor negativo aparecer. LAWSON \& HANSON (1974) indicam a possibilidade do problema ser resolvido através de uma troca simétrica de linhas e colunas de modo que o valor do radical seja maximizado para cada valor de i. Isto postergaria a ocorrência de valores negativos e, quando todos os valores fossem não positivos, as correspondentes linhas restantes de $\mathbf{U}$ seriam anuladas.

Segundo THISTED (1988), todos os cálculos de uma análise de regressão, por exemplo, poderão ser realizados através da decomposição de Cholesky. Pondera, entretanto, que o motivo é mais conceitual do que computacional, uma vez que existem métodos de maior estabilidade numérica, como a decomposição de Householder, e, dentre aqueles que se baseiam no sistema de equações normais, existe o SWEEP, que fornece mais informações para a regressão do que o método de Cholesky.

Para LAWSON \& HANSON (1974), o problema não está no método de Cholesky e sim na necessidade de obtenção do sistema de equações normais para a posterior obtenção da solução de quadrados mínimos. Os mesmos autores fornecem uma ampla discussão comparando a possibilidade de acumulação de erros de arredondamento no método de Cholesky e na decomposição de Householder para o mesmo objetivo sem que o sistema de equações normais necessite ser encontrado, com clara vantagem para este último. 
Na decomposição LU, o processo de inversão da matriz dos coeficientes, como se viu, não é muito eficiente, embora seja muito fácil de ser feito. Tal dificuldade não ocorre na decomposição de Cholesky dada as características da decomposição de uma matriz simétrica. Assim, se L é a triangular obtida através do processo de Cholesky, ela pode ser invertida no lugar e pode-se, a partir daí, chegar facilmente à inversa de A.

\subsubsection{O operador SWEEP e relacionados}

A utilização do operador SWEEP é feita dentro do mesmo contexto da utilização da decomposição de Cholesky. Sofre, portanto, das mesmas desvantagens potenciais já relacionadas para o método de Cholesky, como salientam, dentre outros, THISTED (1988), KENNEDY \& GENTLE (1980), LAWSON \& HANSON (1974) e CHAMBERS (1977). Entretanto, o operador SWEEP, termo introduzido por BEATON (1964), possui muitas vantagens, vantagens que não se limitam à simples economia de memória. É uma variante da eliminação de Gauss-Jordan, mas, em termos estatísticos, é muito superior a ela e não pode ser considerado simplesmente um método de inversão de matrizes.

Segundo GOODNIGHT (1979), o SWEEP é, provavelmente, o mais versátil de todos os operadores estatísticos, podendo ser facilmente adaptado para uso em:

a) - Quadrados mínimos ponderados ou não;

b) - Quadrados mínimos em dois e três estágios;

c) - Quadrados mínimos não lineares;

d) - Análise da variação multivariada;

e) - Todas as regressões possíveis;

f) - Regressão "ridge";

g) - Regressão passo a passo.

h) - Além disso, o SWEEP é uma importante ferramenta educacional, uma vez que em cada passo cada elemento da matriz operada pode ser rapidamente identificado e tem um significado estatístico preciso.

MCCULLAGH \& NELDER (1989), ao discutir algoritmos computacionais para o ajustamento de modelos lineares generalizados, mostram a maior adequação do SWEEP 
ao processo de ajustamento, pois a decomposição de Cholesky e as decomposições do tipo QR (de Householder, Givens e Gram-Schmidt) perdem seus atrativos uma vez que o processo exige que o algoritmo permita iteração de modo eficiente e a inclusão de pesos e da variável dependente ajustada em cada ciclo da iteração.

De uma forma mais simplificada, o passo $\mathrm{k}$ do operador SWEEP sobre a matriz A, que pode ser representado por $\operatorname{SWEEP}(\mathrm{k}, \mathbf{A})$, pode ser traduzido através do algoritmo que segue:

$$
\begin{aligned}
& \text { Passo 1.Para } j=1 \text { até n faça } a_{k j}=a_{k j} / a_{k k} \\
& \text { Passo 2. Para } i=1 \text { até } n, i \neq k \text {, faça } \\
& \text { Passo 3. Para } j=1 \text { até n faça } a_{i j}=a_{i j}-a_{i k} a_{k j} \\
& \text { Passo 4. Faça } a_{i k}=-a_{i k} / a_{k k} \\
& \text { Passo 5. } a_{k k}=1 / a_{k k}
\end{aligned}
$$

Se ao invés da matriz A for considerada a matriz aumentada (15), então, ao se aplicar o SWEEP sobre todas as colunas de A de

$$
\left[\begin{array}{lr}
A & d \\
d^{T} & y^{T} Y
\end{array}\right]
$$

esta se transformará na inversa (se for positiva definida), d será substituído pela solução do sistema de equações normais e sobre o termo inferior direito será colocada a soma de quadrados do resíduo.

Isto, aliado à reversibilidade aparente do operador SWEEP, fornece uma situação muito cômoda quando os cálculos se referem, por exemplo, à retirada ou inclusão de variáveis em modelos, situação extremamente comum na análise de dados. Note-se, entretanto, que a reversibilidade completa somente é possível se A tiver posto completo. Se assim não for, é possível adaptar o algoritmo, tal como faz GOODNIGHT (1979), de modo a contornar a questão da reversibilidade (ela passa a ser feita somente onde é possível). A inversa generalizada (G2) de $\mathbf{A}$ e a forma de Hermite a ela associada são facilmente obtidas como subproduto da aplicação do SWEEP, como mostra o mesmo autor. 
Outra questão importante que aparece associada ao posto completo (ou incompleto) de A é a possibilidade de ocorrer, após a aplicação $\operatorname{SWEEP}(1, \mathrm{~A}), \ldots, \operatorname{SWEEP}(\mathrm{k}-$ 1, A), uma colinearidade quase perfeita entre as $\mathrm{k}-1$ colunas já operadas e a coluna $\mathrm{k}$. O valor da diagonal $\mathrm{k}$, nesse momento, pode ser interpretado como sendo a soma de quadrados condicional da coluna $\mathrm{k}$ em relação às anteriores $\mathrm{e}$, sendo muito próximo de 0 , indica a colinearidade e vice-versa. A simples observação do primeiro passo do algoritmo para o SWEEP indica o efeito catastrófico que isso poderá acarretar se alguma ação não for tomada nessa circunstância. GOODNIGHT (1979) fornece uma ampla discussão acerca desse problema e propõe uma metodologia para a definição de um valor abaixo do qual o pivô será considerado como zero.

\subsubsection{A DVS e relacionados}

A decomposição por valores singulares, DVS, é um resultado fundamental da análise numérica no que diz respeito aos métodos computacionais importantes para a estatística, particularmente no que tange aos modelos lineares. Segundo THISTEAD (1988), a importância da DVS na estatística se dá sob dois ângulos. Primeiro, os valores singulares são a solução para um importante problema variacional, fornecendo uma interpretação estatística direta, a qual, por sua vez, conduz à uma clara compreensão dos modelos lineares e das quantidades a eles associadas. Segundo, a decomposição por valores singulares pode ser utilizada para avaliar a estabilidade numérica de vários algoritmos, quantificando em qual extensão as perturbações existentes nos dados de entrada afetam as saídas, ou seja, os resultados da análise. Isto traduz, portanto, a enorme importância que possui a DVS no contexto da estatística computacional.

A descrição do algoritmo que segue será baseada essencialmente em GOLUB \& REINSCH (1971). FORSYTHE et alli (1977) e LAWSON \& HANSON (1974) também apresentam uma descrição detalhada da metodologia. Uma matriz $\mathbf{A} \mathrm{m} \times \mathrm{n}$, com $\mathrm{m}$ $\geq \mathrm{n}$, pode sempre ser decomposta na forma:

$$
A=U \cdot \Sigma \cdot V^{T}
$$


onde

$$
U^{T} U=V^{T} V=V \cdot V^{T}=I_{n} \quad \text { e } \quad \Sigma=\operatorname{Diag}\left(\sigma_{1}, \ldots, \sigma_{n}\right)
$$

Os elementos da diagonal de $\Sigma$ são denominados de valores singulares e a decomposição (16) é denominada decomposição por valores singulares de A. Em geral, assume-se que esses valores são não negativos e não decrescentes e, se $\mathrm{r}(\mathbf{A})$ é o posto de $\mathbf{A}$, então os valores singulares serão nulos da posição $r(\mathbf{A})+1$ em diante.

A aplicação do algoritmo resume-se nos seguintes passos:

Passo 1. Redução de A a uma forma bidiagonal. Pode ser mostrado que é possível construir duas seqüências finitas de transformações de Householder:

$$
\begin{array}{ll}
P^{(k)}=I-2 X^{(k)} X^{(k) T} & k=1, \ldots, n \\
Q^{(k)}=I-2 y^{(k)} y^{(k) T} & k=1, \ldots, n-2
\end{array}
$$

onde $\mathbf{x}$ e $\mathbf{y}$ são vetores de norma 1, tais que:

$$
P^{(n)} \ldots P^{(1)} \cdot A \cdot Q^{(1)} \ldots Q^{(n-2)}=J^{(0)}
$$

é uma matriz bidiagonal superior. O modo como as matrizes de Householder são construídas é descrito exaustivamente em LAWSON \& HANSON (1974). GOLUB \& REINSCH (1971) descrevem como elas são obtidas no contexto da DVS. Para uma matriz A 5 x 4, por exemplo, a matriz bidiagonal superior é dada por (20).

$$
\left[\begin{array}{cccc}
q_{1} & e_{2} & 0 & 0 \\
0 & q_{2} & e_{3} & 0 \\
0 & 0 & q_{3} & e_{4} \\
0 & 0 & 0 & q_{4} \\
0 & 0 & 0 & 0
\end{array}\right]
$$

Os valores singulares de $\mathbf{J}^{(0)}$ são os mesmos de A. Considerando-se as matrizes de transformação dadas por (21),

$$
P=P^{(1)} \ldots P^{(n)} \quad \text { e } Q=Q^{(1)} \ldots Q^{(n-2)}
$$

então pode-se relacionar a decomposição como em (22): 


$$
\begin{aligned}
& J(0)=G \cdot \Sigma \cdot H^{T} \\
& A=P \cdot G \cdot \Sigma \cdot H^{T} \cdot Q^{T} \\
& A=U \cdot \Sigma \cdot V^{T}
\end{aligned}
$$

Passo 2. Decomposição por valores singulares de uma matriz bidiagonal. A matriz bidiagonal resultante é diagonalizada iterativamente, por meio de rotações de Givens, através de uma variante do algoritmo QR de Francis, denominado "QR de Francis com mudança implícita". LAWSON \& HANSON (1974) fazem uma completa explanação desse passo e fornecem um algoritmo apropriado para a tarefa, o QRBD. GOLUB \& REINSCH (1971) também descrevem com detalhes o processo de obtenção dessas matrizes e como é definido o final do processo de convergência. Resumindo, ter-se-á:

$$
J^{(0)}+J^{(1)}+\ldots \rightarrow \Sigma
$$

onde

$$
J^{(i+1)}=S^{(i) T \cdot J^{(i)}} \cdot T^{(i)}
$$

e as matrizes $\mathbf{S}$ e $\mathbf{T}$ são ortogonais.

Como subprodutos importantes da DVS aparecem a pseudo-inversa ou inversa de Moore-Penrose e a solução de quadrados mínimos de um sistema qualquer, ambos, evidentemente, relacionados. Assim, dada a decomposição de $\mathbf{A}$ através de (16), pode-se provar que a matriz $\mathrm{n} \times \mathrm{m}$ dada por (25), que atende às quatro condições de Moore-Penrose em relação à matriz $\mathbf{A}$. Conseqüentemente, é a inversa de Moore-Penrose de A.

$$
A^{+}=U \cdot \Sigma^{+} \cdot V^{T}
$$

LAWSON \& HANSON (1974) dirigem todo o aparato da DVS para a solução do problema de quadrados mínimos em muitos dos seus aspectos e, por conseqüência, todos os passos têm esse objetivo, particularmente na organização dos resultados dos algoritmos. De qualquer forma, entretanto, o problema de quadrados mínimos consiste na solução de (26), que pode ser expressa através de (26).

Se for exigido que dentre todas as soluções possíveis será escolhida a de menor norma 


$$
\|y-A \cdot x\|_{2}=\min
$$

então a solução será única e não é difícil verificar que:

$$
\hat{x}=A^{+} \cdot y=V \cdot \Sigma^{+} \cdot U^{T} \cdot y
$$

é a solução de norma mínima.

\subsubsection{A decomposição ortogonal e relacionados}

A grande vantagem das transformações ortogonais, no que tange à solução de quadrados mínimos, é não afetar a distância euclidiana sob a multiplicação. Assim, considerando-se a distância expressa através de (26) e a matriz ortogonal $\mathbf{Q}$ tem-se:

$$
\|Q \cdot(y-A \cdot x)\|_{2}=\|y-A \cdot x\|_{2}
$$

A forma de processar a decomposição depende da metodologia escolhida, da dimensão e do posto da matriz A. LAWSON \& HANSON (1974) citam seis casos distintos. De uma maneira geral, entretanto, pode-se dizer que se $\mathbf{A}$ é uma matriz $\mathrm{m} \times \mathrm{n}$ de posto $\mathrm{k}$, então existem matrizes ortogonais $\mathbf{H}, \mathrm{m} \times \mathrm{m}, \mathrm{e} \mathbf{K}, \mathrm{n} \times \mathrm{n}$ tais que:

$$
A=H \cdot R \cdot K^{T}
$$

onde

$$
R=\left[\begin{array}{cc}
R_{11} & 0 \\
0 & 0
\end{array}\right]
$$

e $\mathbf{R}_{11}$ é uma submatriz triangular $\mathrm{k}$ x k não singular. As matrizes $\mathbf{H}$ e $\mathbf{K}$ podem ser escolhidas de tal modo que a riangular seja inferior ou superior.

Além da decomposição por valores singulares, quando se fala em métodos de decomposição ortogonal, usualmente se refere a uma de três metodologias existentes para a sua execução: a reflexão de Householder, a rotação de Givens e a transformação de GramSchmidt. Todas as três possuem uma estabilidade numérica muito boa mas diferenciam-se 
quanto à maior ou menor eficiência computacional, seja quanto à quantidade de memória exigida ou quanto à rapidez. De uma maneira geral, a reflexão de Householder é considerada a mais eficiente, por possibilitar implementações que, ao mesmo tempo, são econômicas e rápidas, embora em aplicações mais particulares uma das outras duas possa ser mais adequada.

LAWSON \& HANSON (1974) quebram a transformação de Householder em duas fases: a construção da transformação e a aplicação da transformação a outros vetores. A construção da transformação de Householder equivale à construção de uma matriz ortogonal Q tal como (31) e aplicá-la a um vetor $\mathbf{v}$ corresponde ao produto Qv.

$$
Q=I_{m}+\frac{u \cdot u^{T}}{b} \quad b=-\frac{\|u\|^{2}}{2} \quad\|u\| \neq 0
$$

Como se pode ver através de (32), o efeito da matriz $\mathbf{Q}$ sobre o vetor $\mathbf{v}$, transformando-o no vetor $\mathbf{y}$, pode ser descrito por meio de três parâmetros inteiros não negativos $\mathrm{p}, \mathrm{l}$ e $\mathrm{m}$, tais que $1 \leq \mathrm{p} \leq \mathrm{m}$ e $\mathrm{p}<1$, qual seja:

a) - Se $\mathrm{p}>1$, os componentes de 1 até p-1 permanecem inalterados.

b) - O componente p é alterado e é chamado de elemento pivô.

c)- $\quad$ Se $\mathrm{p}<1-1$, os componentes de $\mathrm{p}+1$ até $1-1$ permanecem inalterados.

d) - Se $1<\mathrm{m}$, os componentes de 1 até $\mathrm{m}$ são zerados.

Uma questão importante a ser notada na transformação de Householder é a de que a matriz $\mathbf{Q}$ não é formada explicitamente para a aplicação da transformação. A matriz de transformação global feita sobre um conjunto de vetores somente poderá ser obtida através do produto das transformaçōes individuais obtidas para cada vetor. Como mostra LAWSON \& HANSON (1974), dado um vetor $\mathbf{v}$, é sempre possível encontrar um vetor u a partir do qual se pode construir a transformação. O algoritmo computacional que os autores descrevem para a obtenção de u e de b é dado em (33), onde sign indica o sinal do argumento e Q poderá ser construída a partir de u tal como colocado em (31). Os. autores apresentam, ainda, os algoritmos $\mathrm{H} 1$ e H2, respectivamente para construir e aplicar e somente para aplicar a transformação de Householder. Fornecem, também, uma implementação desses algoritmos 
em Fortran.

$$
\begin{aligned}
& Q \cdot v=\left[\begin{array}{c}
v_{1} \\
\vdots \\
v_{p-1} \\
-\sigma\left(v_{p}^{2}+\sum_{i=1}^{m} v_{i}^{2}\right)^{1 / 2} \\
v_{p+1} \\
\vdots \\
v_{1-1} \\
0 \\
\vdots \\
0
\end{array}\right]=y \\
& s=-\operatorname{sign}\left(v_{p}\right)\left(v_{p}^{2}+\sum_{i=1}^{m} v_{i}^{2}\right)^{1 / 2} \\
& u_{i}=0 \quad i=1, \ldots, p-1 \\
& u_{p}=v_{p}-s \\
& u_{i}=0 \quad i=p+1, \ldots, 1-1 \\
& u_{i}=v_{i} \quad i=1, \ldots, m \\
& b=s u_{p}
\end{aligned}
$$

LAWSON \& HANSON (1974) também apresentam a transformação de Givens, apropriada quando se deseja anular somente um elemento do vetor v. A rotação de Givens também é aplicada através de uma série fatorada de transformações individuais fazendo com que exatamente um dos elementos se anule em cada aplicação. Como salienta THISTEAD (1988), ela, em geral, necessita trabalhar mais para executar a mesma tarefa que a de Householder, mas é de grande utilidade em situações onde o número de zeros da matriz a ser operada é muito grande (caso, por exemplo, de matrizes esparsas e de matrizes bi ou tridiagonais, utilizadas na DVS e na determinação de autovalores).

Como somente dois valores de $v$ é que serão alterados, então é suficiente 
considerar a matriz de transformação $G$ sobre o vetor $v$ de dois componentes, como se pode ver nas equações 34 e 35 .

Se os componentes de $v$ não são ambos nulos, então existe uma matriz ortogonal:

$$
\left[\begin{array}{cc}
c & s \\
-s & c
\end{array}\right] \quad c^{2}+s^{2}=1 \quad \text { tq } \quad G \cdot v=\left[\begin{array}{c}
\left(v_{1}^{2}+v_{2}^{2}\right)^{1 / 2} \\
0
\end{array}\right]
$$

Para que esta afirmativa seja verdadeira, basta tomar

$$
\begin{aligned}
& c=\frac{v_{1}}{\sqrt{v_{1}^{2}+v_{2}^{2}}} \\
& s=\frac{v_{2}}{\sqrt{v_{1}^{2}+v_{2}^{2}}}
\end{aligned}
$$

Os mesmos autores também apresentam os algoritmos Gl e G2 (e os implementam em Fortran) responsáveis, respectivamente, pela construção e pela aplicação da rotação de Givens.

Baseados nos algoritmos H 1, H2, Gl e G2, LAWSON \& HANSON (1974) apresentam vários outros algoritmos, visando, dentre outros objetivos, a solução de sistemas de equações de posto completo (HFT), de posto possivelmente incompleto (HFTI, onde implementam também uma estratégia de troca de colunas) e a decomposição (e a análise) por valores singulares. Além disso, mostra estratégias para inclusão e exclusão de variáveis, propõe metodologias para realizar a transformação de Householder em matrizes que não podem ser colocada inteira na memória, etc.

A transformação de Householder não forma uma nova base de modo explícito e sim existe de forma implícita numa série fatorada de matrizes ortogonais. Uma vantagem da transformação ortogonal de Gram-Schmidt é que ela opera no sentido de construir uma nova base ortogonal, que gera o mesmo subespaço gerado pela matriz original, passo a passo, onde cada vetor é construído de modo que seja ortogonal a todos os que já foram construídos. O algoritmo atualmente utilizado para a execução dessa transformação é denominado de método modificado de Gram-Schmidt e é devido à BJÖRCK (1967). O 
algoritmo MGS, tal como apresentado por THISTED (1988), substitui a matriz A n x p pela nova base ortogonal e obtém os valores da triangular superior $\mathbf{R}$. Ele consiste do seguinte:

$$
\begin{aligned}
& \text { Passo 1. Para } \mathrm{j}=1 \text { até } \mathrm{p} \text { faça } I_{j j}=\sqrt{\sum_{i=1}^{n} x_{i j}^{2}} \\
& \text { Passo 2. Para } \mathrm{i}=1 \text { até } \mathrm{n} \text { faça } x_{i j}=x_{i j} / x_{i j} \\
& \text { Passo 3. Para } \mathrm{k}=\mathrm{j}+1 \text { até p faça } r_{j k}=\sum_{i=1}^{D} x_{i j} x_{i k} \\
& \text { Passo 4. Para } \mathrm{i}=1 \text { até n faça } x_{i k}=x_{i k}-x_{i j} I_{j k}
\end{aligned}
$$

THISTED (1988) salienta, ainda, que embora o MGS seja numericamente estável para obter os coeficientes de regressão, problemas poderão acontecer se A for uma matriz mal condicionada. Em algumas situações, poderá ser necessária uma reortogonalização das colunas da matriz "ortogonal" e o MGS poderá ser totalmente inadequado.

\subsubsection{Autovalores e autovetores}

A obtenção dos autovalores e autovetores é feita através de uma metodologia muito parecida com aquela já discutida para a obtenção da DVS. A descrição que segue está baseada principalmente em LEON (1980).

Como os processos de obtenção são todos iterativos, a matriz inicial deve ser transformada de modo a evitar que essa obtenção demande um tempo proibitivo. A redução inicial é feita através de transformações de similaridade por meio de matrizes de Householder. A matriz original $\mathbf{A}$ é transformada na matriz B, que se parece com algo como (36). Essa matriz é denominada "forma superior de Hessenberg", onde $b_{i j}=0$ sempre que $i \geq j$

+ 2. As transformações de similaridade são obtidas através de (37), onde cada $\mathbf{H}_{\mathbf{i}}$ é uma matriz de Householder. 


$$
\begin{aligned}
& {\left[\begin{array}{cccccc}
x & x & \ldots & x & x & x \\
x & x & \ldots & x & x & x \\
0 & x & \ldots & x & x & x \\
0 & 0 & \ldots & x & x & x \\
\vdots & & & & & \\
0 & 0 & \ldots & x & x & x \\
0 & 0 & \ldots & 0 & x & x
\end{array}\right]} \\
& B=H_{n-2} \ldots H_{2} \cdot H_{1} \cdot A \cdot H_{1} \cdot H_{2} \ldots H_{n-2}
\end{aligned}
$$

Note-se que se A for simétrica então $\mathbf{B}$ também será e segue-se que B será tridiagonal.

Uma vez que $\mathbf{A}$ já foi reduzida a uma forma conveniente, o algoritmo QR de Francis, que tem por base a transformação de Givens, poderá ser aplicado de forma eficiente. Definindo

$$
A_{1}=A=Q_{1} \cdot R_{1} \quad \text { e } \quad A_{2}=Q_{1}^{T} \cdot A \cdot Q_{1}=R_{1} \cdot Q_{1}
$$

e fatorando novamente a matriz resultante, obter-se-á uma nova matriz similar à $\mathbf{A}$ da forma

$$
A_{3}=Q_{2}^{T} \cdot A_{2} \cdot Q_{2}=R_{2} \cdot Q_{2}
$$

de onde se pode ver que, em geral,

$$
A_{k+1}=R_{k} \cdot Q_{k}
$$

e, como mostra LAWSON \& HANSON (1974), a seqüência de matrizes converge, sob condições muito gerais, para uma matriz do tipo

$$
\operatorname{Diag}\left(B_{1}, B_{2}, \ldots, B_{s}\right)
$$

onde na diagonal estão submatrizes $2 \times 2$ ou $1 \times 1$. Cada bloco $2 \times 2$ corresponde a um par de conjugados complexos que são os autovalores de $\mathbf{A}$. Se $\mathbf{A}$ for simétrica, então a seqüência irá convergir para uma matriz diagonal com os autovalores reais de $\mathbf{A}$ na diagonal.

Tal como na DVS, o processo de convergência pode ser acelerado através da mudança de origem. Para tal obtém-se no passo $\mathrm{k}$ a constante $\alpha_{\mathrm{k}}$ e procede-se à decomposi- 
ção:

$$
A_{k+1}-\alpha_{k} I=R_{k} \cdot Q_{k}
$$

Essa mudança de origem, entretanto, também poderá ocasionar alguns problemas na obtenção dos autovalores, como se pode ver em PRESS et alli (1989). Na realidade, utiliza-se uma forma que é matematicamente equivalente à (38), mas sem que a mudança de origem seja feita explicitamente. Por outro lado, a matriz $\mathbf{R}$ que aparece na decomposição não é obrigatoriamente triangular superior. Se ela for triangular inferior, a decomposição passa a se chamar QL e o algoritmo aplicado dessa forma sobre uma matriz tridiagonal é denominado QL com mudança implícita.

\subsubsection{A geração de variáveis aleatórias}

A geração de variáveis aleatórias, em geral, pode ser subdividida em duas partes: a geração de variáveis uniformes e a geração de variáveis de outras distribuições utilizando variáveis geradas segundo a distribuição uniforme. Os números uniformes são aqueles números aleatórios cujos valores estão dentro de uma amplitude preestabelecida (geralmente entre 0 e 1) e que a ocorrência de um número em intervalos de mesma amplitude é tão provável quanto em qualquer outra. Os valores de variáveis que seguem outras distribuições são quase sempre gerados através da execução de operações sobre um ou mais valores uniformes. Daí se pode compreender a importância de se ter um bom gerador de números aleatórios uniformes. FORSYTHE et alli (1977) mostram um exemplo de uma rotina que era muito utilizada pois acompanhava a biblioteca do sistema IBM 360, e que, não raro, poderia causar enormes problemas.

Os geradores de valores uniformes podem ser subdivididos em duas partes: aqueles que são fornecidos pela linguagem (ou pelo sistema) e aqueles que são os denominados geradores portáteis, ou seja, poderão ser implementados em qualquer linguagem e, para as mesmas condições iniciais, o seu funcionamento (teoricamente) deve ser o mesmo, qualquer que seja essa linguagem. Para PRESS et alli (1989), os geradores fornecidos pelo sistema são quase sempre do tipo linear congruencial, que geram uma seqüência de números inteiros entre 
0 e m-1 através da relação de recorrência

$$
I_{j+1}=a I_{j}+C(\bmod m)
$$

onde $\mathbf{m}$ é o módulo e a e c são respectivamente, o multiplicador e o incremento. Essa recorrência eventualmente poderá se repetir com um período não superior a $\mathbf{m}$. Uma escolha apropriada de $\mathbf{m}$, a e $\mathbf{c}$ produz um período de comprimento máximo, ou seja, $\mathbf{m}$.

Para PRESS et alli (1989), o gerador linear congruencial tem a vantagem de ser muito rápido, uma vez que trabalha com inteiros e requer somente algumas operações. Suas desvantagens básicas podem ser colocadas segundo dois aspectos: não está livre da correlação serial dentro de um conjunto sucessivo de chamadas e a casualização dos bits de menor ordem não é feita com a mesma eficiência que a casualização dos de maior ordem.

Citando KNUTH (1981) como referência básica, PRESS et alli (1989) implementam várias rotinas para a geração de números uniformes. As técnicas utilizadas são três, basicamente.

A primeira consiste em embaralhar uma seqüência de números gerados através do gerador do sistema (no caso, Random do Turbo Pascal) de modo a quebrar a correlação seqüencial existente. Esse processo, de uma maneira geral, pode ser descrito da seguinte forma:

Passo 1. Gerar uma tabela de $\mathrm{n}$ valores uniformes.

Passo 2. Gerar um novo valor uniforme.

Passo 3. Utilizar o valor gerado no passo 2 para construir um índice $\mathrm{j}$ para acesso à tabela gerada no passo 1 .

Passo 4. Preencher a posição j da tabela com um novo número uniforme.

A tabela do passo 1 será construída toda vez que se quiser inicializar o processo. Caso contrário, ela somente será consultada renovando-se os valores das posições obtidas.

A segunda opção consiste em trabalhar com uma mistura de três geradores portáveis. Um deles é utilizado para a parte (bits) menos significativa do número, um outro é utilizado para a parte mais significativa e um terceiro para controlar uma rotina de 
embaralhamento, absolutamente essencial, segundo os autores, devido ao valor pequeno de $\mathrm{m}$ disponível. O gerador resultante é muito superior a qualquer um dos individuais: seu período é infinito para todos os propósitos práticos e pode-se pensar que não possui nenhuma correlação seqüencial sensível. Para BUSTOS \& ORGAMBIDE (1992), a grande desvantagem de um gerador combinado é que ele é mais lento que qualquer um dos geradores que o define e que, além disso, pouco se conhece acerca das estruturas resultantes da combinação.

A terceira técnica é a implementação de um gerador que não é baseado no método linear congruencial e sim no método da subtração. Como salientam PRESS et alli (1989), a qualidade de um gerador depende, também, das características da aplicação para a qual se quer o gerador. Assim, possuindo geradores de tipos diferentes, as fraquezas de um, se existirem, se devem à motivos muito diferentes das possíveis fraquezas de outro. Assim, se um gerador não for adequado a uma aplicação, um outro poderá servir perfeitamente. BUSTOS E ORGAMBIDE (1992) discutem muitas outras características e sugerem também outros geradores de variáveis uniformes, além de muitos testes para verificação da qualidade de geradores.

A geração de valores de variáveis aleatórias com distribuição distinta da uniforme pode ser feita segundo dois métodos muito gerais: o método da inversão e o da rejeição.

A transformação integral de probabilidade encontrada, por exemplo, em MOOD et alli (1974), baseia-se no seguinte: se X é uma variável aleatória com função de distribuição contínua $\mathrm{F}(\mathrm{x})$, então $\mathrm{U}=\mathrm{F}(\mathrm{x})$ é uniformemente distribuída sobre o intervalo $(0$, 1). Por outro lado, se $U$ é uniformemente distribuído sobre o intervalo $(0,1)$, então $X=F^{-}$ ${ }^{1}(U)$ tem função de distribuição $F($.). Isto significa que, se a função de distribuição de $X$ é conhecida, então valores $\mathrm{x}$ da variável $\mathrm{X}$ com distribuição $\mathrm{F}$ poderão ser encontrados calculandose o valor de $\mathrm{F}^{-1}(\mathrm{u})$, onde u representa um valor de $\mathrm{U}$. Em termos de geração de variáveis aleatórias, basta gerar um valor de uma variável uniforme e substituir na inversa da função de distribuição. O grande problema nesse método é a impossibilidade, em algumas situações, de se obter a inversa de modo razoável, seja analítica ou numericamente.

Segundo PRESS et alli (1989), o método da rejeição é um método poderoso e geral para a geração de valores de variáveis aleatórias cuja função de densidade 
seja conhecida. O método não exige que a função de distribuição seja facilmente calculada e muito menos que a sua inversa seja conhecida. O método da rejeição para alguma distribuição conhecida $p(x)$ exige que se conheça, também, uma função de comparação $f(x)$. A função $f(x)$ deve sempre ser superior a $\mathrm{p}(\mathrm{x})$, inversível e possuir área finita. Os mesmos autores descrevem a aplicação do método da seguinte forma: o método da inversão é utilizado inicialmente para gerar um valor aleatório $\mathrm{x}$ da distribuição $\mathrm{f}$. Um segundo valor uniforme é utilizado para decidir se o valor $x$ será aceito ou rejeitado. Se for rejeitado, um novo valor de $f$ será encontrado e assim por diante. A razão entre pontos aceitos e rejeitados é a razão da área sob $\mathrm{p}$ com a área entre $\mathrm{p}$ e f.

\subsubsection{Probabilidades e pontos percentuais}

MARDIA \& ZEMROCH (1978) apresentam um conjunto completo de procedimentos para a obtenção de tabelas dos valores de variáveis aleatórias com distribuição F e outras distribuições relacionadas como a t, qui-quadrado, normal, gama e beta. No seu trabalho, esses autores apresentam ainda as tabelas para inúmeros valores de graus de liberdade, as técnicas de interpolação e aproximação e os algoritmos, escritos em Algol 60, para o cálculo de áreas e dos valores críticos ou pontos percentuais para cada distribuição. A obtenção dos pontos percentuais é feita, sempre, através do algoritmo auxiliar Hyp, descrito por esses autores, que resolve uma equação geral do tipo $f(x, a, b, \ldots)=0$.

PRESS et alli (1989) apresentam um grande elenco de funções, por eles denominadas funções especiais, dentre as quais se encontram algumas técnicas para a obtenção dos valores das funçōes gama e beta e das funçōes de distribuição da gama (gama incompleta), beta (beta incompleta), F, t e qui-quadrado. Esses autores apresentam, também, procedimentos para a obtenção dos valores das funçōes gama, gama incompleta e beta incompleta. Todas as demais distribuições são obtidas como função dessas. Não apresentam nenhum procedimento para obtenção de valores críticos.

Alguns algoritmos para o cálculo de integrais de probabilidade e suas inversas são também apresentados por COOK et alli (1985) e por DACHS (1988). KENNEDY \& GENTLE (1980) apresentam uma discussão mais técnica acerca dos processos de obtenção 
e uma extensa indicação bibliográfica sobre o assunto. A forma mais conveniente de se tratar o assunto, entretanto, está estreitamente relacionada com a abordagem de PRESS et alli (1989) e MARDIA \& ZEMROCH (1978). Em particular, estes últimos implementam rotinas de cálculo muito genéricas que contemplam vários aspectos importantes nas aplicações, como a ocorrência de graus de liberdade fracionários e a especificação de que lado se deseja a área. Esses últimos autores também oferecem uma extensa relação bibliográfica que dá suporte às suas implementações. 


\section{METODOLOGIA}

O Turbo Vision é uma biblioteca de objetos que inclui, dentre outros, suporte para:

a) - Janelas múltiplas com possibilidade de redimensionamento e sobreposição;

b) - Menus pendurados;

c) - Suporte a mouse;

d) - Caixas de diálogo;

e) - Botões, barras de rolagem, linhas de entrada e de verificação e botões de rádio;

f) - Tratamento padronizado de cliques de mouse e teclas;

g) - Entrada e saída para disco, memória EMS e portas seriais a nível de objeto ao invés de dados;

h) - Listas ligadas a nível de objeto e não de dados.

A metodologia básica utilizada neste trabalho é a programação orientada a objetos utilizando a hierarquia de objetos do Turbo Vision. Como já foi discutido, uma biblioteca orientada a objetos usualmente não é modificada (a não ser em alguns aspectos menores) quando vai ser utilizada. Ao invés disso, ela é estendida através das propriedades de herança e polimorfismo de modo a realizar ações mais específicas. Faz-se, então, necessária a descrição, ainda que sucinta, dessa biblioteca para que as extensões possam ser melhor compreendidas. Uma descrição completa pode ser encontrada no TURBO PASCAL 6.0 TURBO VISION GUIDE ou, mais sucintamente, em O'BRIEN (1991). 


\subsection{Introdução}

O suporte à programação orientada a objetos foi implementado a partir da versão 5.5 do Turbo Pascal. Entretanto, não chegou a entusiasmar, uma vez que preconizava a introdução de uma metodologia revolucionária mas dava poucas ferramentas para a sua utilização. A situação se alterou drasticamente a partir da versão 6.0, com a criação do Turbo Vision. O Turbo Vision, como se verá, é muito mais que uma simples biblioteca, uma vez que redefine quase que todo o suporte dado pelo Turbo Pascal ao programador, a maneira de programar e ainda funciona como um gerenciador das aplicações desenvolvidas sob as suas regras. É necessário dizer que o Turbo Vision é, também, uma biblioteca muito avançada de objetos e, portanto, a sua utilização pressupõe um razoável conhecimento da filosofia e da forma de implementação de objetos. Utiliza, quase que à exaustão, a idéia de polimorfismo e alocação dinâmica de objetos, o que o torna extremamente flexível, mas, ao mesmo tempo, de utilização razoavelmente difícil.

\subsection{A Programação Dirigida a Eventos}

O objetivo básico do Turbo Vision é oferecer ao programador uma estrutura ou um "esqueleto" para que ele o preencha com a "came". Esse "esqueleto" se torna, por assim dizer, o proprietário do programa, fornecendo um ambiente de trabalho completo para aplicações interativas. Dentro desse contexto, além da biblioteca de objetos, uma outra característica marcante do Turbo Vision é o seu suporte à programação dirigida a eventos. Assim, uma aplicação Turbo Vision sempre funciona em resposta aos diferentes tipos de eventos.

Um evento pode ser definido como sendo ocorrências às quais a aplicação deve responder, ou, mais precisamente, são pacotes de informação que descrevem ocorrências discretas às quais a aplicação precisa responder. Tipicamente correspondem a eventos o pressionamento de uma tecla e um clique de mouse. Muitos desses eventos se transformam em comandos, ou seja, estabelecem que tipo de ação a aplicação deve tomar para responder 
àquele evento.

Uma classe de objetos de suma importância dentro da hierarquia do Turbo Vision é aquela que define de que modo uma informação será manuseada numa porção retangular da tela. Cada objeto desse tipo, chamado view, possui um método virtual que gerencia todo o tratamento dos eventos encaminhados a essa view. Como é um método virtual, todos eles podem ser sobrepostos por métodos de descendentes para possibilitar o tratamento de eventos não reconhecidos pelo ancestral e que devem ser tratados por esses descendentes.

\subsection{A Biblioteca de Objetos do Turbo Vision}

Para se ter uma idéia mais precisa da hierarquia de objetos do Turbo Vision, apresenta-se na Figura 1 uma descrição sucinta dos objetos que compõem a biblioteca.

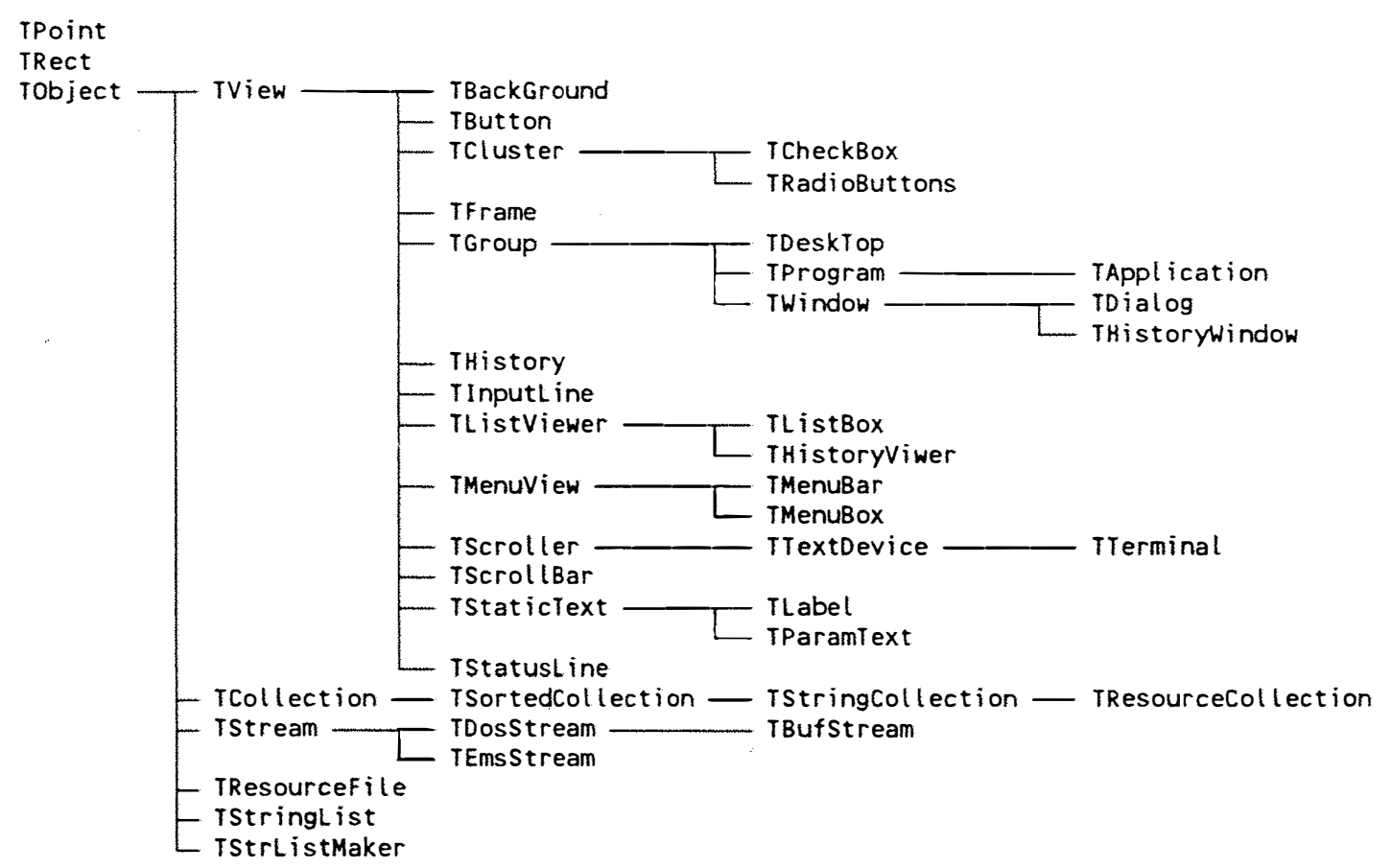

Figura 1. Hierarquia dos objetos do Turbo Vision.

Na árvore hierárquica da Figura 1 pode-se notar que todos os objetos, com exceção de TPoint e de TRect, são descendentes de TObject e a enorme importância do objeto TView, do qual derivam todos os demais objetos que têm algo a fazer através da tela, ou seja, 
que participam da interface do Turbo Vision.

Nem todos os objetos são criados da mesma forma no Turbo Vision. se separá-los em três grupos distintos: objetos primitivos, objetos de interface e objetos mudos. Cada um deles será descrito numa sessão separada. Dentro de cada um desses grupos existem, também, diferentes tipos de objetos, alguns dos quais já são objetos úteis que podem ser utilizados do modo como foram construídos, enquanto que outros são objetos abstratos que servem como base para obtenção de objetos úteis relacionados.

\subsubsection{Objetos primitivos}

O Turbo Vision fornece três tipos de objeto simples que existem primariamente para serem utilizados por outros objetos ou para servirem como base de uma hierarquia mais complexa de objetos. TObject é a base dessa hierarquia, enquanto que TPoint e TRect são utilizados por todos os objetos visíveis da hierarquia do Turbo Vision.

\section{TPoint}

TPoint é simplesmente um objeto que define um ponto na tela. Seus campos $\mathrm{X}$ e $\mathrm{Y}$, definem as coordenadas cartesianas de uma posição na tela. $\mathrm{O}$ ponto $(0,0)$ é o topo mais elevado à esquerda da tela. O campo $\mathrm{X}$ cresce horizontalmente para a direita enquanto que Y cresce verticalmente para baixo. TPoint não possui métodos (logo poderia ter uma estrutura de registro), mas outros tipos possuem métodos que o converte em coordenadas globais (a tela toda) e locais (relativas à origem da view).

\section{TRect}

Este objeto representa um retângulo. Seus campos, A e B, são objetos do tipo TPoint que definem os pontos superior esquerdo (A.X e A.Y) e inferior direito (B.X e B. Y) do retângulo. TRect se parece com um objeto tipo TView, mas apenas fornece os limites superior esquerdo e inferior direito do retângulo e métodos utilitários que não mostram nada na tela. 


\section{TObject}

TObject é um tipo básico abstrato que não possui campos. É o ancestral de todos os objetos do Turbo Vision, exceto de TPoint e de TRect. Os descendentes de TObject caem em duas grandes famílias: a família dos objetos de interface (views) e a família dos objetos mudos. Os objetos de interface são descendentes de TView, que thes fornece propriedades que não são compartilhadas pelos que não são seus descendentes. Os objetos de interface podem desenhar-se a si próprios e tratar eventos que são enviados para ele. Outros objetos que não são visíveis fornecem um conjunto de utilitários para tratamento de streams e coleções de outros objetos, incluindo os de interface, mas eles não são diretamente visíveis.

\subsubsection{Objetos de interface}

Os descendentes exibíveis de TObject são conhecidos como objetos de interface (views) e são derivados de TView, um descendente imediato de TObject. Esses objetos sempre correspondem a uma porção retangular do vídeo.

\section{TView}

O tipo de objeto view deve ser um descendente de TView. O próprio TView é um tipo abstrato representando uma área retangular vazia no vídeo. Possuir TView como ancestral, todavia, assegura que cada objeto derivado possuirá, no mínimo, uma porção retangular no vídeo e um método virtual Draw (forçando todos os descendentes imediatos a fornecerem um método Draw específico).

Muito da programação do Turbo Vision utiliza objetos descendentes de TView mais especializados, mas a funcionalidade de TView está por todo Turbo Vision, de modo que é necessário citar pelo menos alguns dos objetos mais comuns que ele oferece.

\section{- TGroup}

A importância de TView é mostrada literalmente na figura 1. Tudo que é visto numa aplicação Turbo Vision, deriva de algum modo de TView. Mas alguns daqueles objetos visíveis são também importantes por uma outra razão: Eles possibilitam que muitos 
objetos ajam como num concerto.

TGroup possibilita tratar listas ligadas de subviews relacionadas e que se interagem entre si através de uma view chamada de proprietária do grupo. Cada view possui um campo que aponta para a proprietária do objeto TGroup. Um ponteiro nil significa que a view não possui proprietária. Um campo chamado Next possibilita a ligação para a próxima view na cadeia de views. Como um TGroup é também TView, poderão existir subviews donas de suas próprias views e assim por diante.

O estado da cadeia é constantemente alterado por cliques de mouse e pressionamento de teclas durante uma aplicação. Novos grupos podem ser criados e subviews adicionadas (inseridas) ou eliminadas no grupo. Durante o tempo em que está na tela, uma subview poderá estar escondida ou exposta na tela por ações executadas em outras subviews, de maneira que o grupo precisa coordenar muitas atividades.

\section{- TDeskTop}

Desktop é a view de fundo que aparece no início normal, fornecendo uma área de trabalho (desktop) para o usuário, usualmente limitada por uma barra de menus e uma linha de status. Outras views (tais como janelas e caixas de diálogo) são criadas, exibidas e manipuladas na área de trabalho em resposta às ações dos usuários (eventos surgidos através do mouse e do teclado). Muito do trabalho real de uma aplicação se processa dentro dessa área.

\section{- TWindow}

O objeto TWindow, com auxílio do objeto associado TFrame, fornece as áreas com bordas retangulares que podem ser arrastadas, mudadas de tamanho e escondidas utilizando métodos herdados de TView. Pode, ainda, redimensionar-se e fechar-se utilizando os seus próprios métodos.

\section{- TDialog}

TDialog é um descendente de TWindow utilizado para criar caixas de diálogo que tratam de variadas interações com o usuário. Caixas de diálogo tipicamente contem controles tais como botões de rádio e caixas de verificação. 


\section{- TButton}

Um objeto TButton é uma caixa com título utilizado para gerar um evento de comando específico quando ele for "pressionado". Eles usualmente são colocados dentro das caixas de diálogo, oferecendo escolhas tais como OK e Cancela, sendo responsável, portanto, pela transmissão de um comando associado a cada botão.

\section{- TCluster}

TCluster é um tipo abstrato utilizado para implementar caixas de verificação e botões de rádio. Um cluster é um grupo de controles que respondem todos da mesma forma. Os controles do tipo cluster freqüentemente estão associados com os objetos TLabel, conduzindo a seleção por meio de rótulos adjacentes. Possui métodos para o desenho de ícones no modo texto e para marcar os caracteres para acesso rápido.

\section{- TRadioButtons}

Botões de rádio são clusters especiais nos quais somente um controle pode ser selecionado. Cada seleção subseqüente limpa a atual (como acontece com o botão de seleção de uma estação de rádio). Caixas de verificação são clusters nos quais qualquer número de controles podem ser marcados.

\section{- TMenuView}

TMenuView e seus dois descendentes, TMenuBar e TMenuBox, fornecem os objetos básicos para criação dos menus pendurados e submenus hierarquizados em qualquer nível. Necessita somente que lhe seja fornecido cadeias de texto para as seleções de menu (com letras de acesso rápido, opcionais, realçadas) junto com os comandos associados à cada seleção. Os métodos HandleEvent cuidarão dos mecanismos do mouse e/ou do teclado (incluindo as letras de acesso rápido) na seleção do menu. As seleções de menu são exibidas utilizando o objeto TMenuBar, usualmente propriedade de um objeto TApplication. Seleções de menu são exibidas em objetos do tipo TMenuBox. 


\section{- TInputLine}

TInputLine é uma view especializada, um editor básico de cadeias para linhas de entrada. É um objeto de utilização muito simples mas de extraordinária utilidade.

\section{- TListViewer}

O objeto TListViewer é um tipo abstrato através do qual são derivadas exibições de listas de vários tipos, tais como TListBox. Os campos e métodos de TListViewer possibilitam exibir listas ligadas de cadeias com controle sobre uma ou duas barras de rolagem. O tratador de eventos permite a seleção por mouse ou por teclas (destacando) os itens na lista.

\section{- TListBox}

TListBox, derivado de TListViewer, implementa as caixas de listas mais comumente utilizados, como as listas de cadeias que contêm nomes de arquivos. Os objetos TListBox representam listas exibidas de tais itens em uma ou mais colunas com barras de rolagem opcionais. As barras de rolagem horizontais de TListViewer não são suportadas.

\section{- TScroller}

Um objeto TScroller é uma view rolável que serve de portal para outra view maior localizada em segundo plano. O rolamento ocorre como resposta à entradas pelo teclado ou ações em associação com objetos TScrollBar. Os objetos TScrollBar possibilitam o controle vertical ou horizontal.

\section{- TStaticText}

Objetos TStaticText são exibições simples utilizadas para exibir cadeias fixas fornecidos pelo campo Text. Eles ignoram quaisquer eventos enviados para eles. O tipo TLabel adiciona a capacidade de seleção de uma view por meio dele.

\section{- TStatusLine}

O objeto TStatusLine foi construído para exibições de linhas de status e de avisos (ajuda), usualmente na última linha do monitor. A linha de status possui a altura de 
um caráter e comprimento limitado pelo comprimento da tela. O objeto oferece exibições dinâmicas reagindo aos eventos listados pela aplicação. Ítens da linha de status podem ser selecionados por mouse ou por meio de teclas de acesso rápido, assim como os objetos TLabel. Muitas aplicações iniciarão sua operação através dos objetos TMenuBar, TDeskTop e TStatusLine.

\subsubsection{Objetos mudos}

A família dos elementos não visíveis, derivados de TObject, são os streams, arquivos de recursos (resource files), coleções e listas de cadeias.

\section{TStream}

Um TStream é um objeto generalizado para tratamento de entrada e saída. Tipicamente corresponde a um arquivo, quando se fala em DOS e no disco como unidade de saída. Nos arquivos e dispositivos de I/O tradicionais, conjuntos separados de funções devem ser construídos para tratar da extração e da conversão de diferentes tipos de dados. Com os streams do Turbo Vision, pode-se criar métodos polimórficos de I/O tais como Read e Write, que sabem como processar os conteúdos particulares dos seus streams. TStream é um objeto abstrato básico que fornece $\mathrm{I} / \mathrm{O}$ polimórficos para e de um dispositivo de armazenamento. Seus descendentes são:

\section{- TDosStream}

TDosStream é um TStream especializado derivado para implementar streams não buferizados no DOS.

\section{- TBufStream}

TBufStream implementa uma versão buferizada de TDosStream.

\section{- TEmsStream}

Um outro stream especializado, TEmsStream, implementa streams na 
memória EMS.

\section{TResourceFile}

Um arquivo de recursos é um tipo especial de stream implementado por TResourceFile onde objetos genéricos podem ser indexados via chaves tipo cadeia.

\section{TCollection}

TCollection implementa um conjunto geral de ítens, incluindo objetos arbitrários de tipos diferentes. Diferentemente das estruturas tipo array e de conjuntos e listas de linguagens não OOP, uma coleção Turbo Vision permite o dimensionamento dinâmico. TCollection implementa uma coleção polimórfica de objetos possuindo métodos que automaticamente aplica ações a todos os componentes da coleção e localiza elementos com determinadas condições através de métodos repetidores. TCollection serve também de base abstrata para coleções mais especializadas, como as relacionadas abaixo.

\section{- TSortedCollection}

TSortedCollection implementa coleções que são ordenadas por chaves. $\mathrm{O}$ ordenamento é definido por um método virtual e abstrato. Também possui um método que implementa a busca binária na coleção ordenada.

\section{- TResourceCollection}

TResourceCollection implementa uma coleção ordenada de índices fonte utilizados pelos streams de recursos.

\subsubsection{Outros objetos}

Além da coleção padrão de objetos, o Turbo Vision fornece juntamente com os disquetes de distribuição outros objetos importantes no contexto das aplicações. Alguns deles são os seguintes: 


\section{TFileViewer}

TFileViewer não é um objeto padrão do Turbo Vision mas sim um objeto que acompanha a documentação. Seu objetivo é implementar um exibidor de arquivos texto na tela utilizando todos os recursos de janelas múltiplas, redimensionamento, etc.

\section{TFileDialog}

Este é apenas um dos objetos presentes na unit StdDlg cuja finalidade é implementar objetos que realizam a interface com os arquivos em discos.

\subsubsection{Convenções de nome}

O Turbo Vision utiliza prefixos mnemônicos para os seus identificadores. A primeira letra do identificador indica se está se tratando com um tipo objeto, com um ponteiro para ele, dos seus registros de stream ou da sua cor da paleta. Do mesmo modo, existe também uma convenção para a denominação de constantes feita sempre através de um prefixo mnemônico para facilitar a compreensão do seu significado. Na medida do possível tal convenção deve sempre ser seguida nas extensões que forem feitas. Quanto aos tipos, ponteiros, registros de streams e cores da paleta, as conveções são:

- Tipos objeto iniciam com T: TObject

- Ponteiros para objetos iniciam com P: PObject $={ }^{\wedge}$ TObject

- Registros de stream começam com R: RObject

- Cores da paleta começam com C: CObject

A Tabela 3 mostra alguns prefixos de constantes utilizadas pelo Turbo Vision que possuem as mais variadas finalidades. A descrição completa das constantes Turbo Vision poderá ser encontrada no TURBO VISION GUIDE. De especial significado para o Turbo Vision são as constantes de prefixo $\mathrm{cm}$. Elas designam os comandos que realizam o papel cenral da programação dirigida a eventos. 
Tabela 3 - Prefixos de algumas constantes do Turbo Vision

\begin{tabular}{lll} 
Prefixo & Significado & Exemplo \\
\hline $\mathrm{bf}$ & Button flag & bfNormal \\
$\mathrm{cm}$ & Command & cmQuit \\
$\mathrm{co}$ & Collection code & coOverFlow \\
$\mathrm{ev}$ & Event constant & evMouseDown \\
$\mathrm{hc}$ & Help context & hcNoContext \\
$\mathrm{kb}$ & Keyboard constant & kbAltX \\
$\mathrm{mb}$ & Mouse button & mbLeftButton \\
$\mathrm{st}$ & Stream code & stOK \\
$\mathrm{wf}$ & Window flag & wfMove \\
$\mathrm{wn}$ & Window numbers & wnNoNumber \\
\hline
\end{tabular}

\subsection{A Navegação no Turbo Vision}

A navegação dentro do Turbo Vision é a forma como o usuário de um programa com essa interface poderá se locomover entre as subviews que estarão localizadas dentro das views. O padrão de interface segue integralmente o padrão CUA (Commonn User Access). O que será descrito é o comportamento default do Turbo Vision, que poderá eventualmente ser alterado nos objetos descendentes. Uma vez que o Turbo Vision possibilita a implementação de um ambiente multijanelas, é necessário definir views focalizadas e views modais. Uma view focalizada é aquela que no momento está no centro das atenções, ou seja, é aquela onde a próxima ação irá ocorrer enquanto que uma view se torna modal quando. somente nela poderá ocorrer alguma ação. É identificada através de uma mudança de cor em monitores coloridos ou indicando os rótulos através dos caracteres « e » nos monitores monocromáticos. 


\section{- Janelas}

TWindow trata as teclas Tab e Shift + Tab como forma de seleção da subview próxima e anterior, respectivamente, selecionáveis numa janela. Janelas numeradas podem ser selecionadas através da tecla rápida de acesso rápido $\mathbf{A l t}+\mathbf{n}$, onde $\mathbf{n}$ é um número de 1 a 9. Cliques de mouse também possibilitam selecionar uma janela que ainda possui ícones de fechamento e de redimensionamento que podem ser acionados via mouse.

\section{- Caixas de diálogo}

O objeto TDialog trata dos eventos gerados pelo usuário tais como pressionamentos de teclas e cliques de mouse. A tecla Esc é tratada especialmente como uma saída. A tecla Enter seleciona a opção focalizada e inicialmente é tratada como o envio de um comando default cmDefault (usualmente significa que o botão default, tipicamente representado pelo rótulo $\mathbf{O k}$, foi pressionado). Tab e Shift + Tab possibilitam o acesso a subviews.

\section{- Rótulos}

Possui a propriedade na qual a view que contém o texto, conhecida como rótulo, pode ser selecionada por cliques de mouse, teclas de cursor ou teclas de acesso rápido do tipo Alt-letra, se houver essa possibilidade.

\section{- Botões}

Eles usualmente são colocados dentro das caixas de diálogo, oferecendo escolhas tais como OK e Cancel. A caixa de diálogo usualmente é a view modal quando ela aparece, de modo que ela apanha e trata todos os eventos, incluindo os seus botões geradores de eventos, geralmente comandos. O gerenciador de eventos oferece muitas formas de pressionamento de um botão: um clique do mouse no retângulo do botão, teclando a letra correspondente ou focalizando o botão através de Tab ou Shift + Tab. O botão focalizado é selecionado através da tecla Enter. 
- Botões de rádio e caixas de verificação

Tanto nos botões de rádio quanto nas caixas de verificação o acesso pode ser por meio de cliques de mouse, Tab ou Shit + Tab ou de letras de acesso rápido. As teclas de seta podem ser utilizadas para navegação dentro de um cluster. A marcação das caixas de verificação poderá ser feita através da tecla de espaço.

\section{- Menus}

A tecla F10 sempre fará com que a barra de menus se torne a view focalizada (focalizando uma das suas opçōes). As teclas de seta Left e Right poderão ser utilizadas para locomoção à esquerda e à direita e então utilizar ENTER para exibir um conjunto de opções correspondente a uma qualquer da barra. As teclas de seta Up e Down poderão novamente ser utilizadas para posicionamento numa opção qualquer e acionada ENTER para a execução do comando. A tecla Alt + letra (em destaque) na barra de menus ou um clique de mouse lista as opções para aquela escolha da barra de menus e uma delas poderá ser escolhida por um novo clique de mouse ou através da tecla de acesso rápido.

\section{- Listas de histórico}

A lista de histórico é selecionada através da tecla Down e a seleção de um componente da lista poderá ser feito através de setas Up e Down ou de um clique de mouse mais ENTER ou por meio de dois cliques de mouse.

\section{- Linhas de entrada}

Trata todas teclas usuais e as de movimento de cursor (incluindo Home e End). Possibilita inserções e eliminações através da seleção dos modos Insert e Overwrite controlando automaticamente o formato do cursor. O mouse pode ser utilizado para marcar blocos de texto.

\section{- Caixas de lista}

A rolagem dos elementos da lista poderá ser feitá através de cliques de mouse no ícone da barra de rolagem, cliques sobre uma opção ou através das teclas Up e 
Down, PageUp ou PageDown. As teclas Home e End conduzem, respectivamente, ao início e ao fim da lista. A seleção, quando for o caso, poderá ser feita por meio de cliques de mouse ou ENTER quando a opção estiver focalizada.

- Linha de status

Ítens da linha de status podem ser selecionados por cliques de mouse ou por meio de teclas de acesso rápido, tal como os objetos TLabel. 


\section{RESULTADOS}

Os resultados serão apresentados na forma de uma referência de objetos, procedimentos e funções. Os objetos e as rotinas que constam nas seções 4.1 e 4.2 são aqueles considerados mais importantes e estão separados por assunto para uma melhor visualização. Os demais, incluindo outros procedimentos, tipos, constantes, etc., poderão ser encontrados no Apêndice 1, listados em ordem alfabética. Pequenos programas que exemplificam o uso da biblioteca e a construção de uma aplicação Turbo Vision poderão ser encontrados no Apêndice 2.

\subsection{A Referência de Objetos}

Os objetos são apresentados pelo nome e na mesma linha está a indicação da unit à qual esse objeto pertence. Segue-se a indicação da hierarquia referente ao objeto terminando com o primeiro objeto do Turbo Vision que é ancestral do objeto introduzido, pois, como já se discutiu, toda biblioteca orientada a objetos é extendida ao invés de modificada, aproveitando toda a funcionalidade e características presentes nos ancestrais dos quais o objeto em construção se declara descendente. A ascendência completa desse objeto padrão do Turbo Vision poderá ser vista na Figura 1. À indicação da hierarquia segue-se uma breve descrição desse objeto, dos seus campos e dos seus métodos. Note-se que, em princípio, todos os campos são de leitura e escrita mas, se for o caso, para a alteração de alguns deles por alguma rotina exterior ao objeto sempre existirão métodos específicos para isso. Sempre que for necessário, a descrição do método será acompanhada da indicação bibliográfica a partir da qual o método foi adaptado. Os campos e métodos sempre serão descritos através da maneira como são declarados e da finalidade para a qual foi feita a sua implementação. Todos os objetos que 
estão abaixo da hierarquia dos objetos padrões Turbo Vision foram desenvolvidos para este trabalho.

\subsubsection{Arquivo de dados}

TDataFileMaker

CrtData

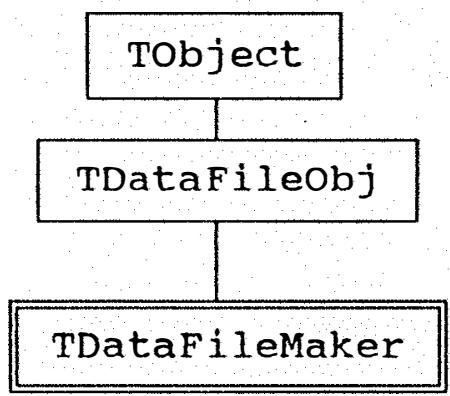

TDataFileMaker é responsável pela criação propriamente dita de um arquivo de dados. Se o arquivo já existe então pode-se incluir registros ou editar os que já existem. Possibilita também a edição da estrutura do arquivo.

\section{Campos}

TextFileName

Declaração

TextFi lentare: PPathStr ;

Finalidade

Ponteiro para uma cadeia (tipo PathStr) que armazena o nome do arquivo texto de onde serão lidos os dados, se for o caso. Se não existir arquivo texto para entrada (a entrada será feita a partir do teclado) aponta para uma cadeia vazia.

RecCol

Declaração 
RecStep

Declaração RecStep: Integer ;

Finalidade RecStep é a quantidade de registros que serão pulados quando a tecla PageDown (para baixo) ou PageUp (para cima) for pressionada durante a edição de um arquivo.

Métodos

Init

Declaração constructor Init (DatafNare, TextfNbre: PathStr; Mode: Wbrd);

Finalidade Init inicializa TDataFileObj, através de DataFName e Mode, e TDataFileMaker atribuindo nil a RecCol, o valor 5 a RecStep e TextFName a TextFileName^. Uma cadeia vazia passada para TextFName indica que a entrada será feita via teclado.

\section{Done}

Declaração destructor Done; virtual;

Finalidade Libera a memória utilizada pelos campos de TDataFileMaker e de TDataFileObj.

Error

Declaração procedure Error (Code: Integer); virtual;

Finalidade Exibe mensagens de erro. Os erros previstos estão na Tabela 4.

Tabela 4. Códigos de erro previstos por TDataFileMaker.Error

Code Descrição

-1 Número de campos mal definido

$-2 \quad$ Número de registros mal definido

-3 Construção de registros mal feita 
Create

Declaração

procedure Create(Mode: Byte);

Finalidade Edita um arquivo de dados no modo estabelecido por Mode. Chama EditStruct para edição da estrutura e InputData para a edição dos registros. Assim, se Mode vale

$\$ 0$ - Não existe o arquivo DataFName e, portanto, serão construídos a coleção de estruturas e os registros através do teclado.

$\$ 1$ - O arquivo DataFName já existe e será feita a edição a partir do primeiro registro.

\$2 - O arquivo DataFName já existe mas o objetivo é a inserção de novos registros. Assim o registro posicionado será o último.

$\$ 3$ - O arquivo DataFName não existe e toda a entrada de dados será feita a partir de um arquivo texto (TextFName).

$\$ 4$ - O arquivo DataFName já existe e a adição de novos registros será feita a partir do arquivo texto TextFName.

InputData

Declaração procedure InputData(CurRec, MbdeEdit: Integer);

Finalidade InputData edita ou insere registros a partir de CurRec. O parâmetro ModeEdit tem o mesmo significado de Mode descrito em Create, que é o procedimento que chama InputData.

ReadTextData

Declaração

procedure ReadTextData;

Finalidade

ReadTextData lê de um arquivo texto todos os dados necessários à construção de um arquivo de dados. A leitura do arquivo texto é feita através do procedimento GetWord, de modo que qualquer caractere presente na constante pré-definida Delim pode ser utilizada como separador de campos. Observe-se, então, que nenhum valor de um determinado campo pode possuir espaços em branco, por exemplo, uma vez que o espaço em branco 
indica à GetWord que a seqüência de leitura já terminou e esse valor retorna como valor do campo.

O arquivo texto poderá ser construído com as definições de campos, registros e comentários em qualquer ordem, desde que os campos e os registros sejam definidos como um bloco. Dados perdidos deverão ser indicados através de um ponto decimal ".".

A palavra Campos indicará que a seguir serão definidos os campos e a palavra Dados indicará que o que segue são os valores dos registros. Seguindo a palavra Campos deve vir (necessariamente) o número de campos e seguindo a palavra Dados deverá vir (necessariamente) o número de registros. Tudo que for distinto de Campos ou de Dados será considerado como comentário. Cada seção de comentários deverá terminar com um espaço em branco e o caractere \} (fecha chave). O espaço em branco é necessário para que GetWord possa retornar somente o caractere \}. À definição dos campos após a palavra Campos e o respectivo número, segue-se a definição de cada campo, ou seja, o nome, o tamanho e o tipo. Se o tipo for $\mathrm{N}$ (campo numérico) deve ser acrescentado o número de decimais para esse campo. Caso contrário, nada mais deve ser incluído para definir esse campo.

Os valores dos campos dos registros deverão ser definidos igualmente logo após a linha que contém a palavra Dados e o número de registros que irão se seguir. Como já comentado, dentre os caracteres que definem o valor de um campo não pode existir nenhum que pertença a Delim.

\section{ReadTextToAppend}

Declaração

procedure ReadTextToAppend;

Finalidade

Semelhante à ReadTextData, com a diferença que não há necessidade de definição de campos, uma vez que os dados presentes no arquivo texto serão incluídos no final de um arquivo já existente. Comentários também são permitidos aqui. 
SetRecStep

Declaração

procedure SetRecStep(AStep: Integer);

Finalidade

Atribui AStep ao campo RecStep.

EditFields

Declaração

procedure EditFields;

Finalidade

Chama EditFields para fazer a edição dos campos e copia para um novo arquivo os registros redefinidos segundo a nova estrutura.

\section{TDataFileObj}

CrtData

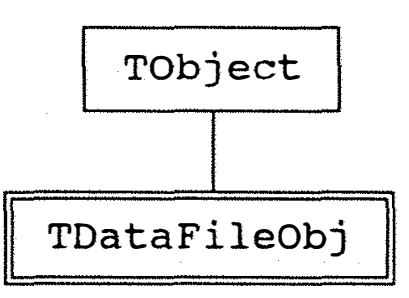

TDataFileObj é um objeto que não é propriamente um arquivo mas está sempre relacionado com um. Seus componentes básicos são um ponteiro para um arquivo de recursos (onde estão os registros, acessados através de chaves numéricas), um ponteiro para um objeto do tipo TObs, que é o registro, e um ponteiro para uma coleção que armazena a estrutura dos registros ou as características de cada campo. Possui dois descendentes que executam tarefas mais especializadas sobre os arquivos tais como a edição de registros e o acesso por condição. Todo arquivo associado à TDataFileObj que for em algum momento modificado terá a forma anterior guardada numa cópia de segurança.

\section{Campos}

Collect

Declaração

Collect: PCollection;

Finalidade

Collect é um ponteiro para uma coleção que armazena as estruturas dos 
campos, tal como descritas em TStruct.

\section{Data}

Declaração

Data: PObs;

Finalidade

Ponteiro para um registro do arquivo de dados. Cada registro possui um campo (tal como discutido em TLStr).

\section{DataFile}

Declaração

DataFile: PResourceFile;

Finalidade

Ponteiro para um arquivo de recursos que armazena a estrutura e os registros. $\mathrm{O}$ registro com chave 0 armazena algumas informações acerca do conteúdo, o número de registros, o número de registros válidos e o tamanho do registro em número de caracteres (ou bytes). Os demais registros possuem estrutura comum, definida nos componentes da coleção apontada por Collect. Note-se, então, que o arquivo apontado por DataFile contém a coleção de estruturas, o registro 0 com informações gerais sobre os registros e os registros de dados (de 1 a NumRecs).

\section{DataFileName}

Declaração

DataFi leNtre: PPathStr;

Finalidade

Ponteiro para o nome do arquivo onde estão os registros.

DataFileOk

Declaração

DataFileOk: Boolean;

Finalidade

Variável lógica que indica problemas no manuseio do arquivo de dados.

\section{NumRecs}

Declaração

NerRecs: Word;

Finalidade

Número total de registros de dados existentes no arquivo em disco. $\mathrm{O}$ 
registro 0 não é considerado, ou seja os registros de dados vão de 1 a NumRecs.

ValidRecs

Declaração ValidRecs: Word;

Finalidade Quantidade de registros válidos existentes no arquivo de dados, ou seja, registros onde o campo IsValid é True. Note-se que os registros marcados ainda estão disponíveis mas não serão utilizados para nenhum efeito prático até que sejam desmarcados.

RecSize

Declaração

RecSize: Word;

Finalidade

Armazena o tamanho do registro, em quantidade de caracteres.

\section{Métodos}

Init

constructor

Init(DataFName: PathStr; Mode: Word);

Finalidade Inicializa todos os ponteiros em nil, NumRecs em 0 e DataFileOk em False. Se DataFName não for uma cadeia vazia e se Mode for diferente de stCreate (constante que indica que um novo arquivo será criado) então abre o arquivo de dados no modo Mode e inicializa o registro na posição zero. Em particular, DataFName é uma cadeia vazia quando se utiliza um descendente de TDataFileObj para acesso às chaves somente, que são armazenadas em um arquivo separado. O parâmetro Mode indica o modo (ou o objetivo) como será aberto o arquivo (se para leitura, para escrita ou para leitura e escrita). Em particular, a constante stCreate indica que a abertura será feita para a criação de um novo arquivo, o que não é feito através de Init: 
Done

Declaração destructor Done; virtual ;

Finalidade Libera toda memória alocada para os campos de TDataFileObj.

Error

Declaração procedure Error(Code: Integer); virtual;

Finalidade Informa sobre possíveis erros ocorridos no acesso aos registros do arquivo de dados. Atribui Code ao campo Status. Neste objeto trata basicamente da abertura com sucesso do arquivo de dados no modo desejado.

InitDataFile

Declaração

procedure InitDataFile;

Finalidade

Inicializa os campos de TDataFileObj com as informações do registro 0 , se for o caso, ou seja, se DataFName não for uma cadeia vazia e se Mode não for stCreate.

PutRec

Declaração

procedure PutRec (Key: hord);

Finalidade Insere no arquivo de dados um registro na posição Key. A chave numérica Key será transformada numa cadeia de duas posições através do procedimento WStr2.

GetRec

Declaração procedure GetRec (Key: Word);

Finalidade Recupera do arquivo um registro com chave numérica Key. A chave numérica Key será transformada em uma cadeia de duas posições por WStr2. 
GetField

Declaração function GetField(Index: Integer): String;

Finalidade Retorna uma cadeia que corresponde ao campo Index do registro apontado por Data.

EditStruct

Declaração

procedure EditStruct (Nm: hord);

Finalidade

Edita a coleção de estruturas apontada por Collect, iniciando no componente Num. Se Num for zero subentender-se-á que uma nova coleção será criada ao invés de editar uma já existente.

GenTST

Declaração

procedure GenTST;

Finalidade

Gera um arquivo texto com os dados presentes no arquivo no formato parecido àquele de onde os dados podem ser lidos de um arquivo texto para a formação do arquivo de dados.

UnMarkRecs

Declaração

procedure UHMrkRecs;

Finalidade

Varre todo o arquivo de dados transformando todo registro marcado como não válido em válido. Note-se que a transformação em registros não válidos é por condição mas a volta é incondicional. A versão anterior do arquivo é guardada como reserva.

\section{DeleteRecs}

Declaração procedure DeleteRecs;

Finalidade Gera um novo arquivo utilizando somente os registros válidos. 
DisposeData

Declaração procedure DisposeData;

Finalidade Libera a porção de memória apontada por Data.

OpenResFile

Declaração function OpenResFile(Nare: PathStr; var ResFile: PResource

File; Mode: hord): Boolean;

Finalidade OpenResFile retorna True se o arquivo Name foi aberto no modo Mode com sucesso. ResFile armazena o endereço do arquivo. Mode possui o mesmo significado já discutido em TDataFileObj.Init.

TFileStruct

FileStru

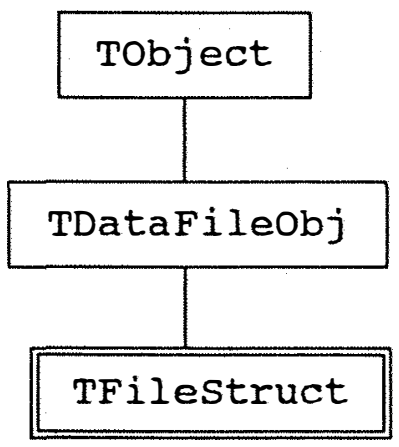

TFileStruct é um descendente de TDataFileObj, responsável por todo acesso à um arquivo de dados através de condições e/ou de chaves. Note-se que todo acesso condicional à um arquivo de dados deve ser feito através de chaves e, portanto, utilizar este objeto.

\section{Campos}

KeyName

Declaração

KevNare: PCollection;

Finalidade

KeyName é um ponteiro para a coleção de nomes dos campos escolhidos para chave. 
KeyCol

Declaração KeyCol: PCollection;

Finalidade KeyCol é um ponteiro para a coleção de registros que contêm os campos chave. Observa-se, então, que os registros de um arquivo de dados é acessado individualmente de onde se pode dizer que não há necessidade de que todos os registros estejam na memória num determinado momento. As chaves, como se pode ver, são armazenadas na forma de uma coleção, gravadas e recuperadas em conjunto, o que significa que todo o conjunto de chaves estarão na memória num determinado momento.

KeyFile

Declaração KeyFile: PResourceFile;

Finalidade KeyFile é um ponteiro para um arquivo de chaves que armazena dois objetos: a coleção de nomes de chaves e os valores das chaves.

KeyFileName

Declaração KeyFileNbre: PPathStr;

Finalidade Ponteiro para uma cadeia (do tipo PathStr) que armazena o nome do arquivo de chaves.

KeyFileOk

Declaração KeyFileOk: Boolean;

Finalidade Vale True se está tudo correto com o arquivo de chaves. Até que o arquivo seja inicializado corretamente vale False.

NumF

Declaração Nemf: Word;

Finalidade NumF é o campo que armazena a dimensão do vetor apontado por Field.

Se o acesso for ao arquivo de dados então NumF valerá Collect^^. Count. Se 
for somente ao arquivo de chaves valerá NumKey.

NumR

Declaração NemR: Word;

Finalidade NumR tem significado semelhante a NumF, mas relacionado ao número de registros. Assim, se o acesso for ao arquivo de dados NumR valerá NumRecs; se for somente ao arquivo de chaves valerá $\mathrm{KeyCol}^{\wedge}$.Count.

$\operatorname{Rec}$

Declaração

Rec: PNArray;

Finalidade

Rec aponta para um vetor fundamental dentro do objeto TFileStruct. Os seus componentes indicam quais serão os registros de dados que serão tratados pelos métodos do objeto. Assim, por exemplo, para a impressão condicional dos registros de um arquivo de dados, a expressão que estabelece a condição é avaliada de acordo com as chaves estabelecidas e o número do registro cujas chaves atendem à condição imposta é satisfeita é transferido para $\operatorname{Rec}^{\wedge}$. Após a avaliação ter sido feita em todas as chaves, o acesso se dá através dos componentes de $\operatorname{Rec}^{\wedge}$. A posição 0 do vetor $\operatorname{Rec}^{\wedge}$ é sempre utilizada para indicar quantos componentes existem dali em diante.

Field

Declaração Field: PNArray;

Finalidade Field tem objetivo idêntico à Rec mas é relacionado com os campos que serão tratados.

Métodos

Init

Declaração const ructor - Init (Dataflare, KeyfNare: PathStr; ModeData, Mode Key: Word);

Finalidade Init inicializa o TFileStruct inicializando os arquivos de dados e de chaves 
(uma cadeia vazia para DataFName ou para KeyFName indica que o arquivo respectivo não é necessário) nos modos ModeData e ModeKey e dimensiona $\operatorname{Rec}^{\wedge}$ e Field^ de acordo com as condiçōes já descritas nos campos NumR e NumF. ModeKey pode assumir dois valores: stCreate, quando o arquivo de chaves será criado ou outra constante stXXXX quando o arquivo será somente consultado. Note-se, portanto, que não há atualização automática dos registros de chaves quando os registros de dados são alterados.

\section{Done}

Declaração

destructor Done; virtual:

Finalidade

Libera toda a memória reservada aos campos de TFileStruct.

Error

Declaração

procedure Error(Code: Integer); virtual;

Finalidade

Emite mensagens de erro no acesso aos arquivos de dados ou de chaves. Os erros previstos são os que constam na Tabela 5

Tabela 5. Códigos de erros reconhecidos por TFileStruct

Code

Descrição

$-1$

Não pode abrir/criar arquivo de chaves

$-2$

Especificação de campo incorreta

$-3$

Não pode criar arquivo de ordenação

$-4$

Não pode abrir arquivo $\mathrm{p} /$ incluir/separar/apagar

$-5$

Número de campos a gerar diferente do definido

$-6$

Todas as chaves devem ser de um mesmo tipo

$-7$

Má definição dos valores de $\mathrm{k}$ e $\mathrm{n}$

$-8$

Não pode criar arquivo.$\$ \$ \$$

$-9$

Especificação incorreta para ordenação

$-10$

Número de registros deve ser o mesmo 
TokenToInt

Declaração

funct ion TokenTolnt (st1: String): Boolean;

Finalidade

TokenToInt retorna True se o preenchimento do vetor Field^ for feito com sucesso a partir da cadeia st1. A indicação dos campos sempre (em todos os locais) deverá ser feita através da letra $\mathrm{x}$ (maiúscula ou minúscula) seguida do número do campo desejado. Assim x1 representa o primeiro campo, $\mathrm{x} 2$ o segundo e assim por diante. Inicialmente GetTokens quebra st1 em pedaços de acordo com a presença dos delimitadores e TokenToInt interpreta cada pedaço da seguinte maneira:

Todos os campos - Indicado com a palavra TODOS. Por exemplo, se existirem 5 campos, o vetor Field^ ${ }^{\wedge}$ para essa indicação terá a seguinte composição [5, 1, 2, 3, 4, 5].

Indicação isolada - A separação deve ser feita através de um delimitador pertencente à Delim. Por exemplo, x1 x10 indica que deverão ser considerados somente o primeiro e o décimo campo e se terá o vetor Field^ ${ }^{\wedge}=[2$, $1,10]$.

Indicação da seqüência - O caractere - poderá ser utilizado para indicar uma seqüência de campos. Para a indicação (no caso de 5 campos) x1, x4o vetor Field^ será $[3,1,4,5]$, enquanto que $\times 2-\times 5$ tornará Field^ ${ }^{\wedge}=[4$, $2,3,4,5]$. Observe que uma indicação de seqüência não poderá conter nenhum delimitador.

\section{CondToInt}

Declaração

procedure CondTolnt (Cond: String);

Finalidade

CondToInt é um procedimento similar à TokenToInt. A diferença é que ele interpreta uma condição imposta aos registros, através de Cond, que deve ser testada através de chaves, para verificar se aquele registro atende a condição. CondToInt utiliza o procedimento TKey.Calculate (que, por sua vez, utiliza StrEval ou FactEval, conforme o tipo de chave) para avaliar a condição imposta na chave correspondente e retornar True ou False. Assim, 
por exemplo, se $x 1$ e $x 4$ de um arquivo de dados são as duas chaves ( $x 1$ e $\mathrm{x} 2$ ) de um arquivo de chaves então listar os registros desse arquivo com a condição $\times 1>\times 2$ implica em listar todos os registros do arquivo onde 0 valor do campo $x 1$ seja superior ao valor do campo $x 4$. Da mesma forma $\operatorname{Rec}^{\wedge}$ armazena na posição 0 a quantidade de registros que atendem à condição estabelecida (e, portanto, que serão listados no exemplo).

\section{SortKeyCollection}

Declaração

Finalidade procedure SortKeyCol lection(s: String);

SortKeyCollection ordena um conjunto de chàves segundo instruções contidas em s. A ordenação é feita em ordem ascendente ou descendente e de forma aninhada para os campos definidos da esquerda para a direita, ou seja, ordena-se inicialmente o primeiro campo. Para os valores repetidos do primeiro ordena-se o segundo e assim por diante. $\mathrm{O}$ algoritmo de ordenação utilizado é o ShellSort e até 50 chaves (colunas do arquivo de chaves) podem ser definidas de cada vez. Assim, por exemplo, se a cadeia $\mathbf{s}$ contivesse os caracteres $A: x 1 d: x 3$ indicaria que a ordenação deve ser feita prioritariamente segundo o campo $\times 1$, em ordem ascendente, e a seguir (em ordem descendente) segundo o campo $\times 3$ (observe-se que $\times 1 \mathrm{e} \times 3$ referem-se às colunas do arquivo de chaves). Como a cadeia $s$ é quebrada por GetTokens, a separação entre duas definições $A: x 1$ e $d: x 3$ deve ser feita por um caractere de Delim. Dentro de uma definição não poderá haver nenhum desses caracteres. A implementação segue os passos definidos em COOK et al (1985) quanto ao algoritmo ShellSort e O'BRIEN (1988) quanto à metodologia de ordenação segundo campos múltiplos. Na realidade não são feitas trocas dos registros de chaves e sim trocas de endereços (ponteiros) dos registros.

GetKeyToRec

Declaração procedure GetKeyToRec; 
Finalidade GetKeyToRec transfere todos os valores do campo TKey.RecNum e os transfere para o vetor $\operatorname{Rec}^{\wedge}$.

MakeKeyFile

Declaração

procedure MakeKeyFile(st: String; Op: Word);

Finalidade Constrói um arquivo de chaves a partir do arquivo apontado por DataFile. A cadeia st indica os campos do arquivo de dados que deverão compor as chaves e Op indica o tipo de chave que será construída no arquivo apontado por KeyFile. Note-se, então, que num arquivo existirá um único tipo de chave. Se forem desejadas chaves de outro tipo outro arquivo deverá ser construído. Tal como já foi comentado, somente será possível construir arquivos de chaves com tipos distintos se todas elas forem indicadas como de tipo StrKey.

Tabela 6. Possíveis valores do parâmetro Op

Op Descrição

$\$ 0$

A chave é do tipo StrKey (cadeia)

$\$ 1$

A chave é do tipo FloKey (número real)

$\$ 2$

A chave é do tipo FacKey (número inteiro)

Observações

A cadeia st deve ser construída do modo como indicado em TokenToInt.

ReadKeyFile

Declaração

procedure ReadKeyFi le;

Finalidade

Carrega o arquivo de chaves do disco e o deixa disponível na memória. Chamada automaticamente por Init sempre que o seu parâmetro KeyFName for uma cadeia não vazia. 
MarkRecs

Declaração

procedure MarkRecs (st1: String):

Finalidade

Marca o campo lsValid dos registros que atendem à condição expressa em st1 com False. A condição stl é avaliada por TKey.Calculate.

SortDataFile

Declaração

procedure SortDataFile(st: String);

Finalidade

SortDataFile gera um arquivo de nome DataFileName^.TSO com os registros (do arquivo apontado por DataFile) ordenados segundo a definição especificada em st.

\section{LinkRecs}

Declaração

procedure LinkRecs (Fi leNbre: PathStr; st: String);

Finalidade

Dados dois arquivos de dados com o mesmo número de registros transfere os campos indicados por st do arquivo FileName para o final dos registros de DataFileName^. Ocorre um erro se o número de registros não for o mesmo. $\mathrm{O}$ arquivo alterado será o apontado por DataFile.

Observações A cadeia st deve ser construída do modo como indicado em TokenToInt.

SepRecs

Declaração

procedure SepRecs (Fi I eNbre: PathStr; st: String);

Finalidade Cria o arquivo FileName cujos campos são os indicados por st. $\mathrm{O}$ arquivo apontado por DataFile não será alterado.

Observações A cadeia st deve ser construída do modo como indicado em TokenTolnt.

GetFloatArray

Declaração

procedure GetFloatArray (var A: PFArray):

Finalidade

Retorna no vetor $A^{\wedge}$ os valores de todos os campos numéricos do arquivo de dados. 
Observações O vetor apontado por A deve ser dimensionado antes que GetFloatArray seja chamada.

GenCol

Declaração

procedure Gericol (st: String);

Finalidade

O objetivo de GenCol é construir novas colunas (campos) no arquivo de dados através de funções das colunas tipo numéricas $(\mathrm{N})$ já existentes. As funções deverão estar definidas em st. Serão geradas tantas colunas quantas forem as funções definidas em st separadas por um delimitador de Delim. Assim, se o arquivo de dados possui quatro colunas e se st for $x 1 * x 2$; $\mathrm{x} 2 / \mathrm{x} 4$, gerará mais duas colunas que serão, respectivamente, o produto da primeira pela segunda e a divisão da segunda pela quarta. $O$ arquivo de dados passará a ter 6 colunas ou campos.

EvallfThen

Declaração

procedure EvallfThen(st, StrThen, StrElse: String);

Finalidade Enquanto GenCol faz uma geração de colunas incondicional, EvalIfThen gera novas colunas de modo condicional. A cadeia st estabelece a condição a ser colocada às chaves. Ao registro que a satisfizer será aplicada a fórmula definida em StrThen; caso contrário a fórmula utilizada será dada por StrElse. Novamente a transformação se refere às colunas numéricas somente.

GenLevels

Declaração

procedure GenLevels (st: String);

Finalidade

A cadeia st deverá ser construída na forma $\mathrm{k}: \mathrm{n}$, onde $\mathrm{k}$ e $\mathrm{n}$ são números inteiros. Este procedimento é utilizado para construir blocos regulares de valores inteiros e com eles construir novas colunas no arquivo de dados. Assim, os $\mathrm{n}$ primeiros valores serão iguais a 1 , os $\mathrm{n}$ seguintes serão iguais 
a 2, e assim por diante. Este processo continua até que o bloco com o valor $\mathrm{k}$ seja construído. A partir desse momento, o processo se repete, com os próximos $\mathrm{n}$ valores sendo iguais a 1, etc., até que todos os valores (NumRec para cada coluna) tenham sido atribuídos. Serão criadas tantas colunas quantas forem as expressões do tipo $\mathrm{k}: \mathrm{n}$ em st separadas por um delimitador de Delim. O algoritmo utilizado está apresentado em BAKER (1986).

\section{DeleteFields}

Declaração procedure DeleteFields(st: String);

Finalidade Eliminar do arquivo de dados os campos definidos através de st.

Observações A cadeia st deve ser construída do modo como indicado em TokenToInt.

\section{ListFields}

Declaração procedure ListFields(s: String);

Finalidade Listar a estrutura do arquivo de dados referente aos campos indicados em s.

Observações A cadeia s deve ser construída do modo como indicado em TokenToInt.

\section{ListRecs}

Declaração

procedure ListRecs(st1, st2: String);

Finalidade Lista os campos definidos por st1 e os registros que atendem à condição estabelecida em st 2 do arquivo de dados no dispositivo indicado em CfgRec para saída.

Observações A cadeia st1 deve ser definida do modo como indicado em TokenToInt enquanto que st2 será avaliado por TKey.Calculate, devendo seguir os critérios de construção já discutidos.

\section{ListKeys}


Finalidade Lista todos os registros correspondentes às chaves do arquivo apontado por KeyFile e indicadas através de st1.

Observações A cadeia st1 deve ser construída do modo como indicado em TokenToInt.

TKey

CrtData

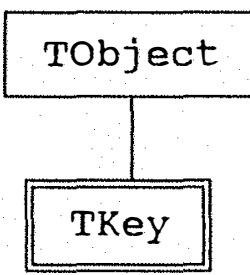

O objeto TKey é responsável por todo o tratamento de chaves para acesso aos arquivos relacionados com o objeto TFileStruct. Como se verá quando se tratar desse objeto, todo o acesso por condição e o ordenamento de arquivos deverá ser feito através de chaves definidas e administradas por TKey. Assim, por exemplo, a ordenação de um arquivo segundo um conjunto de campos envolverá inicialmente a definição daqueles campos como chaves e a ordenação das chaves. $\mathrm{O}$ acesso ao arquivo através das chaves ordenadas produz uma saída na ordem desejada.

Campos

NumKey

Declaração

Nurkey: Byte;

Finalidade

Armazena a quantidade de chaves presentes num dos vetores apontados por Key, NKey ou FKey, ou ainda, é a dimensão desses vetores ou o número de campos considerados como chaves.

DataKey

Declaração

DataKey: KeyType;

Finalidade

Armazena o tipo de chave que no momento está sendo tratada. Como dito anteriormente, existem três tipos de chaves (apenas de um tipo pode ser 
tratado num determinado momento): a chave tipo StrKey (de caracteres), tipo FloKey (campos numéricos do tipo Float) e tipo FacKey (campos numéricos mas com valores inteiros).

Key, NKey, FKey

Declaração

Key: PSt rArray;

Ney: PFArray;

FKey: PNArray;

Finalidade Ponteiros para vetores de chaves tipo cadeia (ponteiro para um vetor de ponteiros), de chaves numéricas de valores reais e de valores inteiros. Notese que qualquer campo tal como será definido em TDataFileMaker poderá ser considerado como do tipo cadeia. Assim, se as chaves escolhidas forem de tipos distintos ela terá que ser necessariamente do tipo Key. Cuidados especiais deverão ser tomados pois, nesse caso, todas as comparações serão feitas através de cadeias.

RecNum

Declaração

RecNem: hord;

Finalidade Armazena o número do registro correspondente às chaves. Esses números serão, de uma maneira geral, repassados a um campo do objeto TFileStruct para que o acesso se processe da forma desejada.

\section{Métodos}

Init

Declaração

constructor Init (Nem: Byte; AKey: KeyType);

Finalidade Init inicializa TKey atribuindo Num a NumKey, AKey a DataKey e dimensiona o vetor de chaves de acordo com esses parâmetros.

Load

Declaração

constructor Load(var S: TStream); 
Finalidade Carrega o objeto TKey do arquivo $\mathbf{S}$ para a memória, inicializando todos os seus campos de forma apropriada.

Done

Declaração destructor Done; virtual;

Finalidade Libera toda memória reservada aos campos de TKey.

Store

Declaração procedure Store(var S: TStream) ;

Finalidade Transfere todo o conteúdo atual dos campos de TKey para o arquivo $\mathbf{S}$.

Calculate

Declaração function Calculate(Expr: String): Boolean;

Finalidade Calculate retorna True se o valor de Expr, avaliada por StrEval, Eval ou

FactEval, conforme o valor de DataKey (ou tipo de chave) for igual a $1 \mathrm{e}$ False se o valor que retorna da avaliação for 0 . Neste contexto, Calculate é utilizado para estabelecer condições de acesso a arquivos relacionados com TFileStruct.

SetKey

Declaração

procedure SetKey (KeyNbre: PString; i: Byte);

Finalidade

Atribui KeyName à i-ésima posição do vetor de chaves. Se Datakey for StrKey o ponteiro KeyName é atribuído diretamente e para DataKey valendo FloKey ou FacKey é atribuído à i-ésima posição o valor de KeyName^.

SetRecNum

Declaração

procedure SetRecNem( $i$ : hord);

Finalidade

Atribui o parâmetro i ao campo RecNum. 


\section{Tobject}

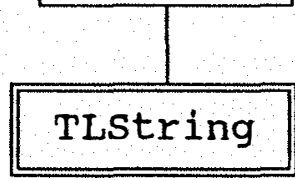

Objeto que implementa a cadeia longa, com todas as facilidades inerentes à um objeto desse tipo: ele sabe dimensionar-se e redimensionar-se de acordo com as necessidades, inicializar-se, inserir e eliminar cadeias, gravarse e recuperar-se, etc. O objeto TLString é uma adaptação de um objeto presente implementação de uma planilha, que acompanha os discos de distribuição do Turbo Pascal versão 6.0.

Campos Len

Declaração Len: LSt ringRange;

Finalidade Este campo armazena a quantidade de caracteres que no momento estão na cadeia longa. É o tamanho efetivo do vetor de caracteres.

MaxLen

Declaração

MaxLen: LStringRange ;

Finalidade

Armazena a quantidade de memória realmente alocada para a cadeia longa. Esse dimensionamento é sempre feito em blocos de 16 bytes para evitar redimensionamentos muito freqüentes.

Data

Declaração

Data: LSt ringDataPtr ;

Finalidade

Ponteiro para o vetor de caracteres, ou seja, é efetivamente o ponteiro para a cadeia longa. 
Métodos

Init

Declaração constructor Init :

Finalidade Inicializa o objeto atribuindo o valor zero a Len e a MaxLen e nil a Data.

Load

Declaração $\quad$ const ructor Load(var S: TSt ream) ;

Finalidade Carrega um objeto TLStr do arquivo $\mathbf{S}$ para a memória.

Done

Declaração destructor Done; virtual; ;

Finalidade Desaloca a memória ocupada por TLStr.

Store

Declaração procedure Store(var S: TSt ream) ;

Finalidade Armazena o objeto TLStr no arquivo S.

SetValue

Declaração funct ion SetValue (Neulen: LStringRange; NewData: Pointer): Boolean;

Finalidade Num objeto TLStr já inicializado, SetValue transfere os NewLen bytes apontados por NewData para o espaço apontado por Data. Se Data for diferente de nil, ou seja, se Data já apontar caracteres, os novos caracteres serão colocados após o último caractere apontado por Data. Set Value retorna True se a atribuição foi feita com sucesso.

Fromitring

Declaração funct ion Frarbtring(S: String): Boolean;

Finalidade

Transforma a cadeia $\mathbf{S}$ em uma cadeia longa através de SetValue. Retorna True se a tarefa for realizada com sucesso. 
ToString

Declaração function ToString: String;

Finalidade Retorna a cadeia longa numa cadeia.

LLength

Declaração funct ion LLength: LSt r ingRange;

Finalidade Fornece o valor atual do campo Len.

Copy

Declaração funct ion Copy (Start, Amt: LStringRange): St ring;

Finalidade Retorna uma cadeia resultante da cópia de Amt caracteres da cadeia longa, iniciando em Start.

Insert

Declaração function Insert(S: String; Start: LSt ringRange): Boolean;

Finalidade Retorna True se a inserção dos caracteres da cadeia $\mathbf{S}$ na cadeia longa, a partir da posição Start, foi feita com sucesso.

Delete

Declaração procedure Delete(Start, Ant: LStringRange);

Finalidade Elimina Amt caracteres da cadeia longa, iniciando em Start. Note-se que a memória ocupada antes da eliminação permanece inalterada.

Append

Declaração function Append(S: String): Boolean;

Finalidade Insere a cadeia $\mathbf{S}$ no final da cadeia longa. Retorna True se a operação foi feita com sucesso. 
Change

Declaração

procedure Change (Ch: Char; Start: LStringRange) ;

Finalidade Substitui o caractere da posição Start da cadeia longa por $\mathbf{C h}$.

Pos

Declaração function Pos (S: String): Word;

Finalidade

Retorna a posição da cadeia longa onde $\mathbf{S}$ inicia. Se retornar zero, então $\mathbf{S}$ não é subcadeia da cadeia longa.

Assign

Declaração

function Assign(LS: TLString): Boolean;

Finalidade

Faz a cópia de uma cadeia longa para outra. Retorna True se a cópia foi realizada corretamente.

TObs

LStr

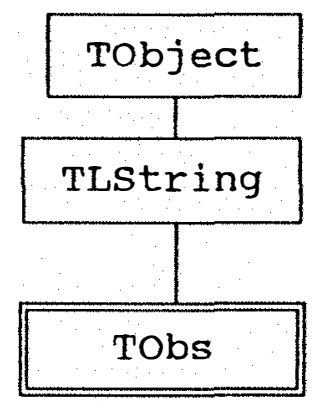

O objeto TObs é um descendente de TLString e tem por finalidade implementar um registro de tamanho variável baseado em caracteres. É o tipo básico de registro para os objetos TDataFileMaker, por exemplo.

\section{Campos}

Number

Declaração

Nmber: Longlnt;

Finalidade

Number armazena o número do registro. 
IsValid

Declaração

IsValid: Boolean;

Finalidade

True se o objeto for válido. Nos objetos TDataFileMaker e TDataFileStruc indica se o registro é válido ou não de acordo com alguma condição.

Métodos

Init

Declaração

constructor Init;

Finalidade

Inicializa primeiramente o ancestral TLStr, atribui zero a Number e True a IsValid, ou seja, em princípio, o objeto é válido.

Load

Declaração

const ructor Load(var S: TSt ream) ;

Finalidade Carrega o objeto TObs do arquivo $\mathbf{S}$ para a memória.

Store

Declaração

procedure Store(var S: TStream);

Finalidade Carrega o objeto TObs da memória para o arquivo $\mathbf{S}$.

SetNumber

Declaração

procedure SetNuber (NenNumber: Longlnt);

Finalidade

Atribui NewNumber ao campo Number.

SetValid

Declaração

procedure SetValid(Valid: Boolean):

Finalidade Atribui Valid ao campo IsValid. 


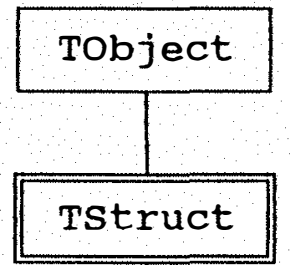

O objeto TStruct é um descendente de TObject e tem por finalidade definir a estrutura dos registros TObs.

\section{Campos}

Name

Declaração

Nare: St rNare;

Finalidade

Name é o nome do campo, limitado a MaxNameSize caracteres.

FieldType

Declaração

FieldType: Char:

Finalidade

Armazena o tipo de campo. Os tipos permitidos são 'C' para campos do tipo alfanuméricos, 'N' para campos numéricos e 'F' para campos tipo fator.

Size

Declaração Size: Word;

Finalidade Size é o tamanho do campo.

Decim

Declaração Decim: Byte;

Finalidade Decim armazena o número de decimais do campo, se ele for numérico.

Offset

Declaração

Offset: Wbrd;

Finalidade

Offset é a posição dentro da cadeia longa onde o campo se inicia. 
MissObs

Declaração Missobs: Byte;

Finalidade

MissObs armazena o número de observações perdidas, identificadas por um ponto ('.') naquele campo.

Métodos

Init

Declaração

constructor Init:

Finalidade

Inicializa o objeto TStruct atribuindo o valor zero aos campos Decim e MissObs.

Load

Declaração

constructor Load(var S: TStream) ;

Finalidade

Carrega o objeto TStruct da memória para o arquivo $\mathbf{S}$.

StrToList

Declaração

function StrToList: String;

Finalidade

Retorna uma cadeia formatada com os valores atuais dos campos de TStruct, para que possa ser impressa a estrutura do arquivo.

Store

Declaração

procedure Store(var S: TStream) :

Finalidade

Armazena o objeto TStruct no arquivo $\mathbf{S}$.

SetName

Declaração

procedure SetName(FieldName: StrName);

Finalidade

Atribui FieldName ao campo Name. 
SetFieldType

Declaração

procedure SetFieldType (Car: Char) ;

Finalidade

Atribui o caractere Car ao campo FieldType.

SetDec

Declaração

procedure SetDec (NmDec: Byte) ;

Finalidade

Atribui NumDec ao campo Decim.

SetOffset

Declaração

procedure SetOffset (Off: Word);

Finalidade

Atribui Off ao campo Offset.

SetSize

Declaração

procedure SetSize(FieldSize: Word);

Finalidade

Atribui FieldSize ao campo Size.

SetMissObs

Declaração

procedure SetMissObs(MO: Byte);

Finalidade

Atribui MO ao campo MissObs.

\subsubsection{Matrizes}

Matrix

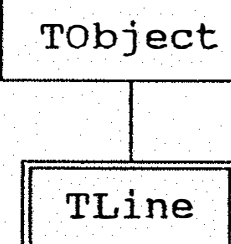

O objeto TLine tem como finalidade definir uma linha para uma matriz. Para 
tanto é dotado de toda a funcionalidade necessária a uma linha: sabe se dimensionar, se inicializar, se aumentar, se carregar da memória para um arquivo (e vice versa), se eliminar da memória, etc. TLine é estreitamente relacionado com TLStr, sendo que cada componente do vetor apontado por Data é um número real (Float) ao invés de um caractere.

\section{Campos}

Len

Declaração

Len: FloatArrayRange;

Finalidade

Len armazena o número de elementos naquela linha.

MaxLen

Declaração

MaxLen: FloatArrayRange;

Finalidade

O dimensionamento do vetor é feito para grupos de 8 elementos de cada vez, ou seja, MaxLen $=($ Len +7$) \operatorname{shr} 2$ shl 2 . Isto evita que sejam feitos novos dimensionamentos a cada ampliação do vetor Data^.

\section{Data}

Declaração

Data: PFÁrray;

Finalidade

Data é um apontador para um vetor de reais (Float) que corresponde a uma linha de uma matriz. Seu dimensionamento é feito sempre que a linha necessite ser inicializada ou aumentada (embora, como já se viu, isto nem sempre seja necessário). O número de bytes alocados (por GetMem) para Data será sempre de MaxLen*SizeOf(Float).

\section{Métodos}

Init

Declaração constructor Init;

Finalidade Inicializa o objeto TLine, atribuindo 0 a Len e MaxLen e nil a Data. 
Load

Declaração constructor Load(var S: TSt ream) ;

Finalidade Carrega o objeto TLine do arquivo $\mathrm{S}$ para a memória.

Done

Declaração destructor Done; virtual:

Finalidade Libera a memória alocada para TLine.

Store

Declaração procedure Store(var S: TStream) ;

Finalidade Armazena TLine no arquivo $\mathbf{S}$.

SetValue

Declaração function SetValue (Nenlen: FloatAr rayRange; Nendata: Pointer): Boolean;

Finalidade SetValue retorna True se o campo Data foi corretamente inicializado a partir do vetor apontado por NewData que contém NewLen componentes. Em outras palavras, SetValue transfere NewLen componentes de NewData ${ }^{\wedge}$ para Data $^{\wedge}$. Note-se que Data ${ }^{\wedge}$ é dimensionado para receber os componentes de NewData^ e este vetor não é alterado.

LLength

Declaração

funct ion LLength(Nem: FloatAr rayRange): Word;

Finalidade

Retorna a quantidade de bytes que serão ocupados pelos Num componentes, ou seja, retorna Num*SizeOf(Float).

\section{Copy}

Declaração

funct i on Copy (Start: Fl oatAr rayRange; var Amt: FloatAr rayRange) : PFAr ray;

Finalidade

Retorna um ponteiro para um vetor de reais correspondente aos Amt 
elementos de Data^ iniciando em Start.

Insert

Declaração function Insert(F: PFArray; FLen, Start: FloatArrayRange): Boolean;

Finalidade Insere no vetor Data^a partir da posição Start os FLen elementos de $\mathbf{F}^{\wedge}$.

Delete

Declaração procedure Delete(Start, Amt : FloatAr rayRange):

Finalidade Elimina de Data Amt componentes iniciando por Start.

Append

Declaração function Append (F: PFAr ray; FLen: FloatAr rayRange): Bool ean;

Finalidade Anexa ao vetor $\mathrm{Data}^{\wedge}$ os FLen componentes do vetor $\mathrm{F}^{\wedge}$.

\section{TMatCol}

Matrix

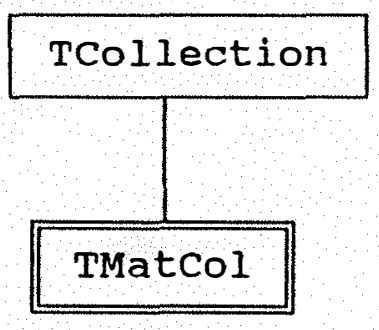

TMatCol tem por objetivo criar uma coleção de matrizes. Seus métodos são capazes de ler e armazenar um conjunto de objetos TMatrix, listá-los, etc.

Métodos

AtM

Declaração

function AtM(k: Word): FMatrix;

Finalidade

AtM retorna um ponteiro para um objeto TMatrix localizado na posição $\mathbf{k}$. 
Printall

Declaração

procedure PrintAll (var W: PMArray);

Finalidade

PrintAll lista, através de TMatrix.Print, todas as matrizes da coleção. Em $W^{\wedge}[0], W^{\wedge}[1]$, etc., deverão estar as dimensões dos campos para as colunas de cada matriz.

ReadTextFile

Declaração

procedure ReadTextFile(var FNare: PathStr);

Finalidade

ReadTextFile toma o caminho definido em FName, lê e carrega na memória, através de ReadMat, todas as matrizes definidas no arquivo texto até que a palavra FIM seja encontrada.

\section{TMatrix}

Matrix

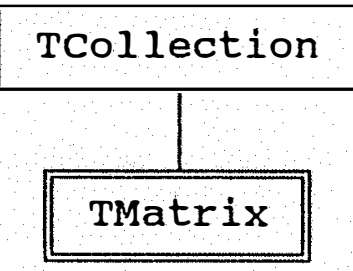

TMatrix é um descendente de TCollection cuja finalidade é construir e manipular uma coleção de objetos TLine, que constitui, cada um, uma linha de uma matriz. A sua funcionalidade, como se verá, é dada por um conjunto de métodos que são responsáveis por ações executadas sobre os elementos apontados pelo campo Data de TLine. Quando o retorno for feito através de uma matriz ela, via de regra, será dimensionada pelo método chamado. O objeto básico de operação será chamado de matriz A, ou seja, os métodos e os campos dizem respeito à esta matriz. Assim, por exemplo, o método MatCopy(var B: PMatrix) será descrito como uma cópia da matriz A na matriz B. Para maior facilidade de descrição será denominado matriz o ponteiro que aponta para uma matriz. Desse modo, o ponteiro B do tipo PMatrix do exemplo citado será denominado matriz $\mathrm{B}$. 
Campos

MatLab

Descrição

MatLab: PString;

Finalidade

MatLab é um ponteiro para uma cadeia cuja finalidade é armazenar um rótulo para a matriz.

MatDec

Descrição

MatDec: Byte;

Finalidade

MatDec armazena o número de casas decimais que serão utilizadas nas saídas envolvendo a matriz.

MatRows

Descrição

MatRons: Word;

Finalidade

MatRows é o número de linhas da matriz.

Observações

O número de linhas na realidade é dado através do campo Count de TCollection. Ocorre que em alguns casos (na matriz tipo diagonal) o armazenamento dos elementos da diagonal é feito através de uma linha somente. Assim Count sempre será igual a $1 \mathrm{em}$ matrizes desse tipo enquanto que MatRows será o número de linhas (lógico) da matriz.

MatCols

Descrição

MatCols: hord;

Finalidade

É o número de colunas da matriz.

MatType

Descrição

MatType: Char:

Finalidade

Armazena o tipo da matriz, G, para matrizes gerais, $S$ para matrizes simétricas, $D$ para matrizes diagonais e $T$ para matrizes triangulares inferiores. 
RowName

Descrição

Rontre: PStrCol lect ion;

Finalidade

RowName é um ponteiro para uma coleção de cadeias que armazenam rótulos para as linhas.

ColName

Descrição

Colntre: PStrCol lection;

Finalidade

Semelhante à RowName, armazenando rótulos para as colunas.

Métodos

Init -

Descrição

constructor Init (AType: Char; nr, nc: Word);

Finalidade

Inicializa o objeto TMatrix atribuindo AType para MatType, nr para MatRows e nc para MatCols, 2 para MatDec e nil para todos os ponteiros. Inicializa TCollection chamando TCollection. Init indicando nr componentes se MatType for diferente de D e 1 se for igual. Se a matriz precisar aumentar seu número de linhas irá fazê-lo de $10 \mathrm{em} 10$ linhas.

Load

Descrição

constructor Load(var S: TSt ream) ;

Finalidade

Recupera o objeto TMatrix armazenado no arquivo $\mathbf{S}$.

Done

Descrição

destructor Done; virtual;

Finalidade

Libera toda a memória ocupada por TMatrix e seus ascendentes.

Store

Descrição

procedure Storè(var S: TSt ream);

Finalidade

Carrega o objeto TMatrix da memória para o arquivo $\mathbf{S}$. 
Error

Descrição procedure Error (Code, Info: Integer); virtual;

Finalidade Procedimento que se sobrepõe a TCollection.Error. Sua finalidade é repassar ao campo Status de TCollection o valor Code, indicando uma possível condição de erro numa operação efetuada pelos diferentes métodos. Os códigos repassados estão na Tabela 7.

Tabela 7. Códigos de erro do objeto TMatrix

Status Descrição

\begin{tabular}{ll}
-1 & Índice da coleção fora da amplitude permitida \\
-2 & Dimensionamento insuficiente para a coleção \\
-3 & Erro na leitura dos parâmetros no arquivo texto \\
-4 & Operação imprópria - pivô igual a zero \\
-6 & Não convergiu nas iterações desejadas \\
-7 & Erro na triangulação de Householder \\
-8 & Modo não permitido na triangulação de Householder \\
-9 & Dimensão imprópria para a operação \\
-10 & Modo de armazenamento não reconhecido \\
-11 & No de nomes diferente do n de linhas/colunas \\
-12 & Tipo impróprio para operação \\
-13 & Modo de armazenamento deve ser $\mathrm{G}$ \\
-14 & Modo de armazenamento deve ser $\mathrm{S}$ \\
\hline
\end{tabular}

SetLabel

Descrição procedure SetLabel (Alabel: String);

Finalidade A cadeia Alabel passa a ser apontada por MatLab.

SetDec

Descrição procedure SetDec (Decim: Byte); 
Finalidade

Atribui Decim a MatDec.

SetName

Descrição

procedure SetNare (st: string; FNare: Boolean);

Finalidade

SetName inicializa ou anexa os nomes contidos em st na coleção de nomes de linhas, se RName for True ou na coleção de nomes de colunas, caso contrário. O parâmetro st é tratado por GetTokens de modo que a separação entre um nome e outro deve ser feita através de um dos delimitadores de Delim.

Print

Descrição

procedure Print (CoMidth: Byte); virtual;

Finalidade

O procedimento Print é o responsável pela impressão da matriz A no dispositivo desejado. Toda a configuração é feita através da variável prédefinida Conf. Os diretórios e os dispositivos de saída são os mesmos já comentados quando essa variável foi descrita. O espaço utilizado por cada coluna é o estabelecido no parâmetro ColWidth, se ele for superior a 4, espaçamento utilizado se for menor. O número de colunas impressas dependerá do dispositivo de impressão, do valor de ColWidth e de Conf.Width. Conf.Size (escrita compactada ou não) também interfere no número de colunas se a impressão for feita na impressora.

\section{MatToText}

Descrição procedure MatToText (var Flare: PathStr; CoMidth: Byte);

Finalidade MatToText transfere toda a matriz A (escrita do mesmo modo como é lida por ReadMat) para o arquivo texto FName utilizando colunas de largura ColWidth. 
AtR

Descrição

function AtR(k: Integer): PLine;

Finalidade

AtR retorna um ponteiro para a linha $\mathbf{k}$ da matriz $A$.

MatCopy

Descrição

procedure MatCopy (var B: ANatrix):

Finalidade MatCopy produz uma cópia da matriz A e coloca na matriz apontada por B.

SortCol

Descrição

procedure SortCol (var Indx: PhArray);

Finalidade

SortCol re-arranja as colunas da matriz A de acordo com o vetor de inteiros Indx, ou seja, a primeira coluna da matriz será a coluna Indx^[1], a segunda Indx ${ }^{\wedge}[2]$ e assim por diante.

Observações $\quad \mathrm{O}$ vetor Indx deverá ser dimensionado e preenchido antes que SortCol seja chamado e o valor Indx^[0] deverá indicar o número de componentes que vem a seguir.

El

Descrição

function El(i, j: Word): Float;

Finalidade

El retorna o valor do elemento localizado na posição $(i, j)$ da matriz $A$, ou seja, linha i e coluna j. El evidentemente leva em consideração o tipo de matriz para retornar o valor.

Mult

Descrição procedure Milt(var B, C: PMatrix; ToC: Boolean);

Finalidade Mult é o procedimento que executa o produto entre a matriz $\mathrm{A}$ e a apontada por B. O resultàdo será armazenado em $\mathrm{C}$ se ToC for True. Caso contrário, o resultado substituirá a matriz A. Essa segunda opção faz com que a matriz 
A se perca mas economiza memória pois não existe a necessidade de dimensionar a matriz resultante, apenas um vetor de dimensão MatCol que irá substituir cada linha que não será mais utilizada na matriz A.

\section{ElemOp}

Descrição

procedure Elemop(var B, C: PMatrix; OpType: Char; ToC: Boolean);

Finalidade ElemOp realiza a operação elemento a elemento entre a matriz A e B colocando o resultado em $\mathrm{C}$ se ToC for True. Caso contrário, a matriz resultante substitui a matriz A. O parâmetro OpT ype indica o tipo de operação que deve ser realizada. Assim, os caracteres + (mais), - (menos), * (asterisco) representam, respectivamente, a soma, a subtração e o produto entre os elementos das matrizes, feito elemento a elemento.

Scalar

Descrição

procedure Scalar (k: Float; OpType: Char);

Finalidade

Scalar executa a operação OpType do escalar k com a matriz A. OpType poderá ser *, /, +, -, respectivamente, produto, divisão, soma e subtração de $\mathrm{k}$ à cada elemento da matriz.

SubMat

Descrição procedure SutMat (var B: ANatrix; 1, nb, k, nb: Word);

Finalidade SubMat coloca em B a submatriz de A. com mb linhas, começando em I, e $\mathbf{n b}$ colunas, começando em $\mathbf{k}$, ou seja, a partir de $\mathbf{l}$ copia $\mathbf{m b}$ linhas e a partir de $\mathbf{k}$ copia $\mathbf{n b}$ colunas.

Rowlnc

Descrição procedure Rowlnc (var B: FMatrix);

Finalidade Anexa em A todas as linhas da matriz apontada por $\mathbf{B}$. 
Collnc

Descrição procedure Collnc(var B: FMtrix);

Finalidade $\quad$ Anexa em A todas as colunas da matriz apontada por $\mathbf{B}$.

Transp

Descrição procedure Transp(var B: AMtrix):

Finalidade Armazena em B a transposta de A.

ATranspA

Descrição procedure ATranspA(var B: AMatrix);

Finalidade Cria uma matriz apontada por $\mathbf{B}$, resultante do produto da matriz A transposta por ela própria.

AATransp

Descrição procedure AATransp(var B: PVatrix);

Finalidade Semelhante à anterior, mas o produto é da matriz A pela sua transposta.

ATranspB

Descrição

procedure ATranspB(var B, C: AMatrix);

Finalidade Efetua o produto da matriz A transposta pela matriz apontada por $\mathbf{B}$ e 0 resultado é colocado numa matriz apontada por $\mathbf{C}$.

ABTransp

Descrição

procedure ABTransp(var B, C: AMbtrix);

Finalidade Produto da matriz A pela transposta de B. O resultado é uma matriz apontada por $\mathbf{C}$.

GenCol

Descrição procedure GenCol (st: string): 
Finalidade $\quad$ Gera novas colunas em A resultantes de expressões matemáticas envolvendo as colunas existentes. As regras para a construção das expressões são as mesmas já discutidas na descrição do procedimento Eval. O número de expressões enviadas através de st indica o número de colunas a serem geradas. Note-se que cada expressão que compõe st deve ser antes tratada por StdForm, StdFunc e FindGenError. Isto já é feito diretamente por TGenInputLine. As expressões a serem analisadas podem ser quebradas através de GetTokens mas deve-se cuidar que as expressões sejam separadas uma da outra através de um caractere de Delim (desse modo, a presença de espaços em branco, por exemplo, em st irá indicar que ali termina uma expressão e começa outra).

GaussJordan

Descrição

procedure GaussJordan (var

B: FVatrix; ToB: Booleanl:

Finalidade

Se ToB for True então o procedimento GaussJordan soluciona um sistema quadrado de equações lineares, através do método de GaussJordan, armazenando em $\mathbf{B}^{\wedge}$ a solução. Na entrada, B deve apontar para a matriz que armazena os vetores do segundo membro da equação em cada coluna. A resolução é feita independentemente para cada vetor. Na matriz A dos coeficientes é armazenada a matriz inversa. Se ToB for False então somente a matriz inversa será determinada e armazenada em A. A implementação é uma adaptação da de PRESS et al (1989).

CholeskyFat

Descrição

procedure CholeskyFat (var r: Word, eps: Float),

Finalidade CholeskyFat promove a fatoração de Cholesky numa matriz A simétrica e positiva semidefinida. Na variável r retorna o posto (numérico ou pseudoposto) da matriz e o parâmetro eps deve repassar a precisão desejada para a determinação do posto. A matriz simétrica A é transformada numa matriz triangular inferior. A implementação segue COOK et al (1985). 
Observações A matriz A que será fatorada por CholeskyFat deve ser e estar armazenada como matriz simétrica.

CholeskySol

Descrição

procedure CholeskySol (var X: AMatrix);

Finalidade

CholeskySol resolve o sistema simétrico de equações lineares. Na matriz simétrica A deverá ser armazenada a matriz dos coeficientes e nas colunas de $\mathbf{X}$ os vetores do segundo membro correspondentes à cada sistema de equações. Antes de CholeskySol a matriz A deve ser fatorada via CholeskyFat. A soluçã̃o é feita através da solução de sistemas triangulares para frente e para trás tal como descrito em LAWSON \& HANSON (1974) e em PRESS et al (1989).

Cov

Descrição

procedure Cov;

Finalidade

O procedimento Cov determina a matriz inversa de A a partir da triangular inferior gerada por CholeskyFat. A inversão da triangular inferior é feita segundo algoritmo proposto por LAWSON \& HANSON (1974).

\section{LUDecomp}

Descrição

procedure LlDecamp(var Indx: PNArray; var d: Float; eps: Float);

Finalidade

LUDecomp aplica o algoritmo LU com pivoteamento parcial na matriz quadrada geral A, efetua a fatoração produzindo uma matriz triangular inferior e uma triangular superior, ambas armazenadas em $\mathrm{A}$. $\mathrm{O}$ vetor de inteiros Indx retornará as trocas de linhas realizadas, $\mathbf{d}$ retornará um valor necessário apenas quando o determinante de $\mathrm{A}$ for desejado e eps indica a precisão com a qual se deseja declarar um pivô como zero. A implementação dessa e das demais rotinas associadas à decomposição LU seguiu a implementação de PRESS et al (1989).

Observações $\quad \mathrm{O}$ vetor de inteiros Indx^ deverá ser dimensionado de acordo com o número 
de linhas (ou de colunas) da matriz antes da chamada de LUDecomp.

\section{LUBackSubst}

Descrição

procedure LUBackSubst (var Indx: PNAr ray; var B: AVBtrix);

Finalidade

LUBackSubst resolve o sistema quadrado de equações lineares sendo $\mathrm{A}$ a matriz dos coeficientes e B a matriz dos vetores do segundo membro, tal como descrito nos métodos GaussJordan e CholeskySol. A solução de cada sistema é armazenada na coluna correspondente de B. Antes que LUBackSubst seja chamado, a matriz A deve ser fatorada através de LUDecomp.

\section{LUInverse}

Descrição

procedure LUInverse (var Y: AMatrix; var Indx: PMArray);

Finalidade

LUInverse encontra a inversa da matriz geral A que é armazenada em $Y$. Como se pode ver não é um processo de inversão "no lugar", como faz, por exemplo, o método de GaussJordan. A matriz é invertida coluna a coluna e é utilizado mais um vetor de dimensão MatCols para a realização do processo. A matriz A deve ser operada por LUDecomp antes de ser invertida e Indx é o vetor de inteiros que retorna de LUDecomp.

\section{LUDet}

Descrição

procedure LUDet (var Indx: PAArray; var d: Float);

Finalidade

LUDet encontra o determinante da matriz A que retorna em d. Como nos demais casos da decomposição LU, LUDecomp deve ser chamada antes e Indx^é o vetor que retorna dessa chamada.

SVDecmp

Descrição

procedure SDeamplvar D, V: AMbtrix; Wat: Boolean; eps: Float; var $r$ : hord):

Finalidade SVDecmp faz a decomposição de A por valores singulares. Desse modo, $\mathrm{A}=\mathrm{UDV}^{\mathrm{T}}$ sendo que $\mathrm{U}$ é a matriz dos autovetores de $A A^{\mathrm{T}}, \mathrm{D}$ é a matriz 
diagonal dos valores singulares e $\mathbf{V}$ é a matriz dos autovetores de $\mathrm{A}^{\mathrm{T}} \mathrm{A}$, calculada se VMat é True. O parâmetro eps deve ser uma constante positiva e pequena de modo que eps vezes o maior valor singular deverá ser a precisão desejada para considerar um autovalor nulo. No parâmetro r retorna o número de valores singulares não nulos ou o pseudo-posto de $\mathrm{A}$. Os valores singulares são ordenados do maior para o menor e as colunas de $U$ e V são reordenadas de modo correspondente. $A$ implementação teve por base o algoritmo proposto por GOLUB \& REINSCH (1971) e descrito e implementado também por FORSYTHE et al (1977). Todas as demais implementações relacionadas são também baseadas nessas referências.

SVDSol

Descrição

procedure SLSol (var Y: PVatrix; eps: Float):

Finalidade

Resolve, baseado na decomposição por valores singulares, o sistema de equações do qual A é a matriz dos coeficientes e $Y$ é a matriz que contém os vetores do segundo membro e eps é o valor que idêntico ao de SVDecmp, que é chamado dentro de SVDSol.

MoorePenrose

Descrição

procedure MorePenrose (var B: PMatrix; eps: Float);

Finalidade

Retorna em B a matriz inversa de Moore-Penrose, calculada a partir da SVD. O parâmetro eps deve ter o mesmo valor já considerado para SVDecmp.

\section{EigenStruct}

Descrição

Finalidade

procedure EigenStruct (var B: FNatrix, EgV: Boolean; eps: Float; var $r$ : hord);

Dada uma matrizsimétrica A, EigenStruct obtém os autovalores e, para EgV

True, os autovetores associados. Os autovalores retornam na matriz diagonal

B e os autovetores, se forem calculados, ocupam o lugar de A. O parâmetro 
eps indica o valor abaixo do qual um autovalor será considerado nulo e em r retorna o número de autovalores não nulos. Todas as rotinas relacionadas a autoestrutura de uma matriz quadrada seguem a implementação de PRESS et al (1989).

Observações A matriz A deve ser simétrica mas armazenada no modo geral, uma vez que vai ser substituída pela matriz dos autovetores.

TQLI

Descrição

procedure TOll(var d, e: PFArray; EgV: Boolean);

Finalidade TQLI é o método chamado por EigenStruct para aplicar o algoritmo QL sobre uma matriz tridiagonal com mudança implícita. Os vetores d e e armazenam os elementos acima e abaixo da diagonal, respectivamente. Em d retornam os autovalores da matriz que é utilizado por EigenStruct para construir a matriz B. EgV indica (True) se a matriz de autovetores vai ser construída.

Tred2

Descrição

procedure Tred2(var d, e: PFAr ray; EgV: Boolean);

Finalidade

O método Tred2 obtem a matriz tridiagonal a partir de A através de transformações de Householder, cujos elementos acima e abaixo da diagonal são armazenados em d e em e. Passando True para EgV os autovetores serão construídos. É automaticamente chamada por EigenStruct.

HesQR

Descrição

procedure HesoR(var B: FMatrix; Maxlts: Word);

Finalidade

HesQR aplica o algoritmo QR numa matriz de Hessenberg visando encontrar os autovalores de uma matriz quadrada não simétrica. Na primeira linha de B estará a parte.real dos autovalores enquanto que na segunda estará a correspondente parte imaginária. Os valores estão ordenados segundo os 
valores da parte real. Para Maxits deve ser passado o número máximo de iterações permitidas para a convergência do processo.

Hessenb

Descrição procedure Hessenb;

Finalidade Hessenb transforma a matriz A numa forma de Hessenberg através de transformações ortogonais de HouseHolder. A forma de Hessenberg será posteriormente tratada por HesQR para obtenção dos autovalores da matriz não simétrica $\mathrm{A}$.

Balanc

Descrição procedure Balanc;

Finalidade Balanc aplica um algoritmo de balanceamento à matriz $\mathrm{A}$ não simétrica da qual serão obtidos os autovalores. Esse balanceamento não é uma operação obrigatória mas normalmente acelera o processo de convergência para a obtenção dos autovalores.

$M G S$

Descrição

procedure MES(var B: FMatrix; ToB: Boolean);

Finalidade MGS transforma a matriz A através do Método Modificado de GramSchmidt. Se ToB for True, a matriz triangular resultante da decomposição será construída e armazenada em B. A implementação segue o algoritmo proposto por LAWSON \& HANSON (1974).

Helmert

Descrição procedure He Imert ;

Finalidade Constrói a matriz de Helmert. 
House

Descrição

procedure House(Build: Boolean; Mode, p, l, m, ia: hord; var up: Float; var C: PMatrix; ncb, ncp: Word);

Finalidade House constrói e opcionalmente aplica a transformáção de Householder, segundo algoritmo proposto por LAWSON \& HANSON (1974), à mätriz A. Se Build é True então a transformação ortogonal é construída e aplicada sobre a matriz $\mathbf{C}$ indicada; caso contrário a transformação (já construída) será somente aplicada a $\mathbf{C}$. A construção da transformação será sempre na matriz A, com o pivô sendo uma sua coluna ou linha. A transformação pode ser aplicada tanto à própria matriz $\mathbf{A}$ quanto à matriz $\mathbf{C}$, aplicada do vetor ncb até o vetor ncp. Se a aplicação for sobre A então $\mathbf{C}$ deverá constar como parâmetro mas não será alterada. O parâmetro ia indica qual o vetor (linha ou coluna) de A que servirá para construir a transformação, p é o índice do elemento pivô, l é a posição a partir da qual os elementos do vetor serão zerados e m é o número de componentes do vetor. O parâmetro Mode é que indicará se a transformação será construída a partir de uma linha ou de uma coluna e se será aplicado a A ou a C (linha ou coluna). Por fim o parâmetro up retorna o componente $\mathbf{p}$ do vetor de transformação $u$. Os valores de Mode na Tabela 8 indicam as possibilidades.

Tabela 8. Modos de aplicação da transformação de Householder Mode Descrição

Coluna ia de A e aplicação sobre colunas de ncb a ncp de A.

Coluna ia de A e aplicação sobre linhas de ncb a ncp de A.

Coluna ia de A e aplicação sobre colunas de ncb a ncp de C.

Coluna ia de A e aplicação sobre linhas de ncb a ncp de C.

Linha ia de A e aplicação sobre colunas de ncb a ncp de A.

Linha de A e aplicação sobre linhas de ncb a ncp de A.

Linha de A e aplicação sobre colunas de ncb a ncp de C.

Linha de A e aplicação sobre linhas de ncb a ncp de C. 


\section{MakeGiv}

Descrição

procedure MakeGiv(v1, v2: Float; var c, s, r: Float);

Finalidade

$\mathrm{O}$ método MakeGiv constrói a rotação de Givens. A entrada consiste nos componentes $\mathbf{v} \mathbf{1}$ e $\mathbf{v} \mathbf{2}$, que são os dois componentes da matriz que serão alterados. A saída consiste dos escalares $\mathbf{c}$ e $\mathbf{s}$, para a aplicação da transformação de Givens e de $\mathbf{r}$, que armazena a raiz quadrada da soma de quadrados de $\mathbf{v} \mathbf{1}$ e v2. O algoritmo de Givens (construção e aplicação) segue a implementação de LAWSON \& HANSON (1974).

AppGiv

Descrição

procedure AppGiv(c, s: Float; var z1, z2: Float);

Finalidade

AppGiv é responsável pela aplicação da transformação de Givens. A entrada consiste dos escalares c e s, que retornam de MakeGiv. A saída se dá através de $\mathbf{z 1}$ e de $\mathbf{z 2}$ que são os componentes da matriz alterados pela transformação.

HFTSol

Descrição

procedure HFSol (var B, X: FMatrix; var H: PFArray);

Finalidade HFTSol resolve, por meio de transformações de Householder, sistemas de equações lineares simultâneas resultantes do método dos mínimos quadrados dos quais $\mathbf{B}$, na entrada, é a matriz cujas colunas são os vetores do segundo membro. Na matriz $\mathbf{X}$ retornam a solução e as somas de quadrados do resíduo correspondente a cada solução enquanto que em $\mathbf{B}$ retorna, nas colunas correspondentes, os resíduos do ajustamento de mínimos quadrados de cada sistema. A pressuposição desse método é de que o sistema é de posto completo (para todas as colunas) e nenhum teste de verificação é feito. Do mesmo modo, não é executada nenhuma troca de linhas ou de colunas. No vetor $\mathbf{H}$ retornam os valores do parâmetro up definido no método House. A matriz triangular resultante da transformação de Householder é armazena- 
da na porção triangular superior de A. A implementação segue o algoritmo HFT proposto por LAWSON \& HANSON (1974).

Observações A matriz $\mathbf{X}$ é definida e inicializada por HFTSol mas o vetor $\mathrm{H}$ de dimensão MatCols precisa ser dimensionado antes da chamada.

\section{HFTISol}

Descrição

procedure HFISol (var B, X: Avatrix; var H, G: PFArray; eps: Float; var $r$ : Word; var Indx: PMrray);

Finalidade

HFTISol tem finalidade semelhante à HFTSol. A diferença é que é capaz de tratar sistemas de posto incompleto aplicando também um algoritmo de troca de colunas enquanto resolve o sistema. A matriz $\mathbf{B}$ na entrada possui os vetores do segundo membro e na saída possui a solução do sistema nas posições de 1 até MatCols para cada coluna (os demais elementos não possuem significado que interesse). A matriz $\mathbf{X}$ possuirá na saída 2 linhas e tantas colunas quantas são as de $\mathbf{B}$, armazenando na primeira as somas de quadrados do resíduo e na segunda as normas da solução. Os vetores $\mathbf{H ~ e}$ $\mathbf{G}$ armazenam respectivamente os valores de up (de House) quando a transformação é construída para ser aplicada à esquerda e à direita. $\mathrm{O}$ parâmetro eps é o responsável pela determinação do pseudo-posto, que retorna em $\mathbf{r}$, da matriz $\mathrm{A}$, ou seja, é o valor segundo o qual todos os valores de mesmo índice da matriz $\mathrm{A}$ operada à esquerda pelas transformações de Householder são considerados nulos se forem menores que ele. No vetor Ind $\mathbf{x}^{\wedge}$ retorna o roteiro das trocas de colunas feitas durante o pivoteamento. A implementação é uma adaptação do algoritmo HFTI proposto por LAWSON \& HANSON (1974).

Observações A matriz $\mathbf{X}$ é inicializada por HFTISol mas $\mathbf{G}$ e $\mathbf{H}$ devem ser dimensionados antes da chamada. Ambos devem ter dimensão MatCols. 
Finalidade A aplicação da transformação de Householder gera a matriz triangular superior $R$ armazenada na porção superior direita de $A$. A matriz $\left(R^{T}\right)^{-1}$ é, afora uma constante (a variância), a matriz de covariâncias da solução. HFTCov determina essa matriz no local onde $\mathbf{R}$ está armazenada. Esse método pode ser chamado depois de HFTSol ou depois de HFTISol. No primeiro caso não existe o vetor de inteiros $\mathbf{P}$ e, portanto, Perm deve ser False. No segundo caso, HFTCov somente pode ser chamada se r (o pseudoposto, que retorna de HFTISol) for igual ao número de colunas de A. Nesse caso, $\mathbf{P}$ é o ponteiro para o vetor de inteiros que retorna de HFTISol e Perm deve ser True. O algoritmo básico é o proposto por LAWSON \& HANSON (1974).

RevSweep

Descrição

procedure Revineep (k: word; DMin: Float; var v: PIArray);

Finalidade RevSweep executa o SWEEP reversível sobre a coluna $\mathbf{k}$ de uma matriz simétrica. O parâmetro DMin é o parâmetro de tolerância para identificação de pivôs muito próximos de zero. O vetor $\mathbf{v}^{\wedge}$ é composto de 1's e -(menos) 1 's. Se a coluna $\mathbf{k}$ é operada pelo sweep então o $\mathbf{k}$-ésimo componente de $\mathbf{v}^{\wedge}$ tem o seu sinal alterado. Esse vetor é fundamental para as demais rotinas que utilizam o sweep, uma vez que mantém a trilha das colunas já operadas e compensa as diferenças de sinais existentes na porção triangular inferior e superior de A provocadas pelo modo de armazenamento. $\mathrm{O}$ algoritmo para implementação do Sweep e outros relacionados são adaptações dos algoritmos sugeridos por GOODNIGHT (1979).

Observações A matriz A deve ser e estar armazenada como uma matriz simétrica.

SweepTol

Descrição procedure SneepTol(var F: PFArray; eps: Float); virtual;

Finalidade Retorna através de $\mathbf{F}$ um vetor de número reais (Float) que poderão servir como níveis de tolerância para a execução de RevSweep. Tal como está 
definido, atribui eps a todos os MatCols componentes do vetor.

Sweep

Descrição

procedure Sneep (var Col: PMArray; var v: PIArray; eps: Float);

Finalidade

Executa RevSweep para todas as colunas indicadas por Col. Na entrada, $\mathbf{v}^{\wedge}$

é preenchido com 1's e retorna a trilha das colunas operadas e eps é o valor da tolerância que será informado a SweepTol. Na chamada de RevSweep, é processada a coluna $\mathrm{Col}^{\wedge}[\mathrm{k}]$ de A e será passado para DMin o componente $\mathrm{Col}^{\wedge}[\mathrm{k}]$ de $\mathrm{F}^{\wedge}$.

Observações $\quad \mathbf{O}$ vetor $\mathbf{v}^{\wedge}$ deve ser dimensionado antes da chamada de Sweep, do mesmo modo que o vetor $\mathrm{Col}^{\wedge}$, que deve ser dimensionado e preenchido antes que Sweep seja chamado. Na posição 0 de $\mathrm{Col}^{\wedge}$ deverá ser colocado o número de colunas que serão operadas pelo sweep.

$G 2 \ln v$

Descrição procedure GQInv(var G: PMatrix; var v: PIArray);

Finalidade G2Inv retorna através de $\mathbf{G}$ a inversa $\mathbf{G} 2$ da matriz A simétrica. Deve ser chamada depois da execução de Sweep seqüencial (componentes de $\mathrm{Col}^{\wedge}$ de 1 a MatCols) sobre A.

Observações A matriz $\mathrm{G}$ é definida por G2Inv.

Hermite

Descrição

procedure Hermite(var H: PMatrix; var v: PIArray);

Finalidade Idêntico a G2Inv, com a diferença que em $\mathbf{H}$ retorna a forma de Hermite associada à matriz simétrica $\mathrm{A}$.

Observações A matriz $\boldsymbol{H}$ é definida por G2Inv. 


\subsubsection{Geração de variáveis aleatórias}

\section{TRandNum}

RNum

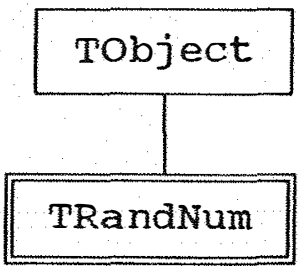

TRandNum é o objeto responsável pela geração de valores de variáveis aleatórias com distribuição uniforme no intervalo $[0,1]$. Todos os métodos de TRandNum foram implementados a partir de algoritmos adaptados de PRESS et al (1989).

\section{Campos}

RanVec

Declaração

RanVec: PFArray;

Finalidade

Ranvec é um vetor de reais utilizado pelas rotinas de geração de uniformes. É dimensionado através do parâmetro de Init.

Lx

Declaração

Ix: PLIntArray;

Finalidade

Vetor de inteiros para uso geral dos métodos.

$\operatorname{Ran} Y$

Declaração

RanY: Float ;

Finalidade

RanY serve a alguns métodos para o armazenamento de um valor a ser utilizado numa próxima etapa. É utilizado também por Ran0 para provocar o embaralhamento no processo de geração de uniformes. 
VecSize

Declaração VecSize: Integer ;

Finalidade VecSize armazena a dimensão do vetor RanVec.

fail

Declaração fail: Integer ;

Finalidade O campo fail armazena um inteiro que é um código de erro. Os códigos possuem o significado colocados na Tabela 9.

Tabela 9. Condições de falha no objeto TRandNum

fail

Descrição

0

Nenhuma condição de erro.

1

Índice inválido para RanVec em Ran0 ou Ran1.

2

Tamanho do vetor não pode ser inferior a 55 em Ran3.

3

Valor ilegal de parâmetro na geração da exponencial ou da gama.

\section{Métodos}

Init

Declaração

constructor Init(vs: Integer);

Finalidade

Init inicializa o objeto dimensionando os vetores RanVec e Ix e fazendo fail igual a zero. RanVec é dimensionado para acolher vs valores de ponto flutuante (Float). Observe que vs deve ter um valor mínimo para alguns geradores.

Done

Declaração

destructor Done; virtual;

Finalidade

Libera toda a memória alocada para RandNum. 
RanO

Declaração function RanO(var idum: Integer): Float;

Finalidade Retorna um valor uniforme entre 0 e 1 utilizando o procedimento Random do Turbo Pascal e utilizando a técnica do embaralhamento da saída. Qualquer valor negativo para idum reinicializa ou inicializa a seqüência e faz RandSeed (variável pré-definida do Turbo Pascal) igual a idum. O vetor RandVec pode ser dimensionado com 97 posições, embora isso não seja o mais importante.

Ranl

Declaração function Ran1(var idum: Integer): Float ;

Finalidade Ran1 implementa outro gerador linear congruencial que é composto da mistura de três geradores. Qualquer valor negativo para idum provoca a inicialização do processo. O vetor RandVec também pode ser dimensionado com 97 posições.

Ran3

Declaração

function Ran3(var idum: Integer): Float:-

Finalidade

Ran3 é o terceiro gerador de uniformes oferecido por TRandNum. Ao contrário dos anteriores, utiliza o método da subtração para gerar os valores uniformes. O vetor RandVec deve ter dimensão 55 e esse valor não pode ser alterado. Um valor negativo para idum provoca a inicialização do processo. 


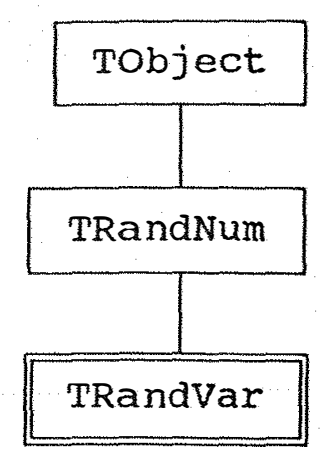

TRandVar é um objeto cuja finalidade é implementar geradores de variáveis aleatórias de algumas distribuições de probabilidade utilizando valores gerados segundo a distribuição uniforme. O método virtual URand estabelece o gerador uniforme utilizado em todos os métodos geradores. Tal como em TRandNum, a metodologia utilizada é a proposta por PRESS et al (1989).

\section{Campos} $x x$

Declaração $x x$ : PFAr ray ;

Finalidade $\mathrm{O}$ vetor $\mathrm{xx}$ é dimensionado internamente com tamanho 6 e é utilizado como área de trabalho para os métodos, particularmente para armazenar resultados que serão posteriormente utilizados em novas chamadas dos métodos.

Métodos

Init

Declaração const ructor Init(vs: Integer);

Finalidade Init inicializa TRandNum (e RandVec através de vs) e dimensiona xx com 6 posições.

\section{Done}

Declaração destructor Done; virtual;

Finalidade Libera toda memória utilizada por TRandVar e TRandNum. 
URand

Declaração function URand(var idum: Integer): Float; virtual ;

Finalidade URand é um método virtual pré-definido para utilizar como gerador TRandNum. Ran3. Pode ser sobreposto para a utilização de um outro gerador qualquer (Random, do Turbo Pascal, por exemplo). O parâmetro idum tem o significado já discutido.

Norm

Declaração

function Norm(var idum: Integer; var Extra: Boolean): Float;

Finalidade

Norm gera valores independentes de uma variável aleatória com distribuição normal de média 0 e variância 1 . Um valor negativo para idum provoca a inicialização do processo de geração da uniforme. Para Extra igual a False o processo é inicializado e gera dois valores independentes. Um deles retorna através de Norm e o outro é armazenado para a próxima chamada e Extra se torna True. Utiliza o método de Box-Miller modificado de modo a evitar o cálculo de funçōes trigonométricas.

\section{Expon}

Declaração

function Expon(var idum: Integer; alfa: Float): Float;

Finalidade

Gera o valor de uma variável aleatória exponencialmente distribuída com média alfa utilizando o método da inversão.

\section{Gamma}

Declaração

function Ganma(var a, idum: Integer): Float;

Finalidade

Retorna um valor gerado de acordo com a distribuição gama de ordem inteira a, isto é, um tempo de espera para o a-ésimo evento num processo de Poisson de média unitária. Utiliza o método da rejeição. 
Poisson

Declaração function Poisson(xm: Float; var idum: Integer; var Chgełn: Boolean): Float;

Finalidade Gera, segundo o método da rejeição, um número de ponto flutuante como um valor inteiro que é o valor de uma variável aleatória retirado de uma distribuição de Poisson de média xm. Sempre que o processo for inicializado ou quando a média xm tiver seu valor alterado, ChgeXm deve ser True.

\section{Binom}

Declaração

function Binomlpp: Float; $n$ : Integer; var idum: Integer; var ChgeN, ChgeP: Boolean): Float;

Finalidade Retorna como um número de ponto flutuante um valor inteiro que é o valor de uma variável aleatória retirado de uma distribuição Binomial de $\mathbf{n}$ experimentos cada um com probabilidade pp. Sempre que o processo for inicializado ou quando a pp e/ou $\mathbf{n}$ tiverem seus valores alterados, ChgeN e/ou ChgeP deverão ser correspondentemente alterados para True. A metodologia utilizada é também o método da rejeição.

A Figura 2 mostra a mesma árvore de descendência mostrada na Figura 1, agora com a adição dos novos objetos descritos na seção 4.1 .

\subsection{Referência das Rotinas Externas à Biblioteca de Objetos}

Muitos componentes importantes da biblioteca não são objetos e sim funções e procedimentos que, evidentemente, não fazem parte da hierarquia de objetos. A descrição dessas funções e procedimentos é feita de modo semelhante à descrição dos objetos, indicando o seu nome, em qual unit está localizada, como foi declarada e qual é a sua finalidade. Do mesmo modo, as indicações bibliográficas, quando necessárias, são feitas junto com a descrição da finalidade da função ou do procedimento. 
Do mesmo modo que na seção 4.1 , somente serão descritas as rotinas mais importantes, localizadas por assunto, sendo que as demais poderão ser encontradas no apêndice A. Exemplos de utilização do material descrito nesta seção poderão ser encontrados no Apêndice 2.

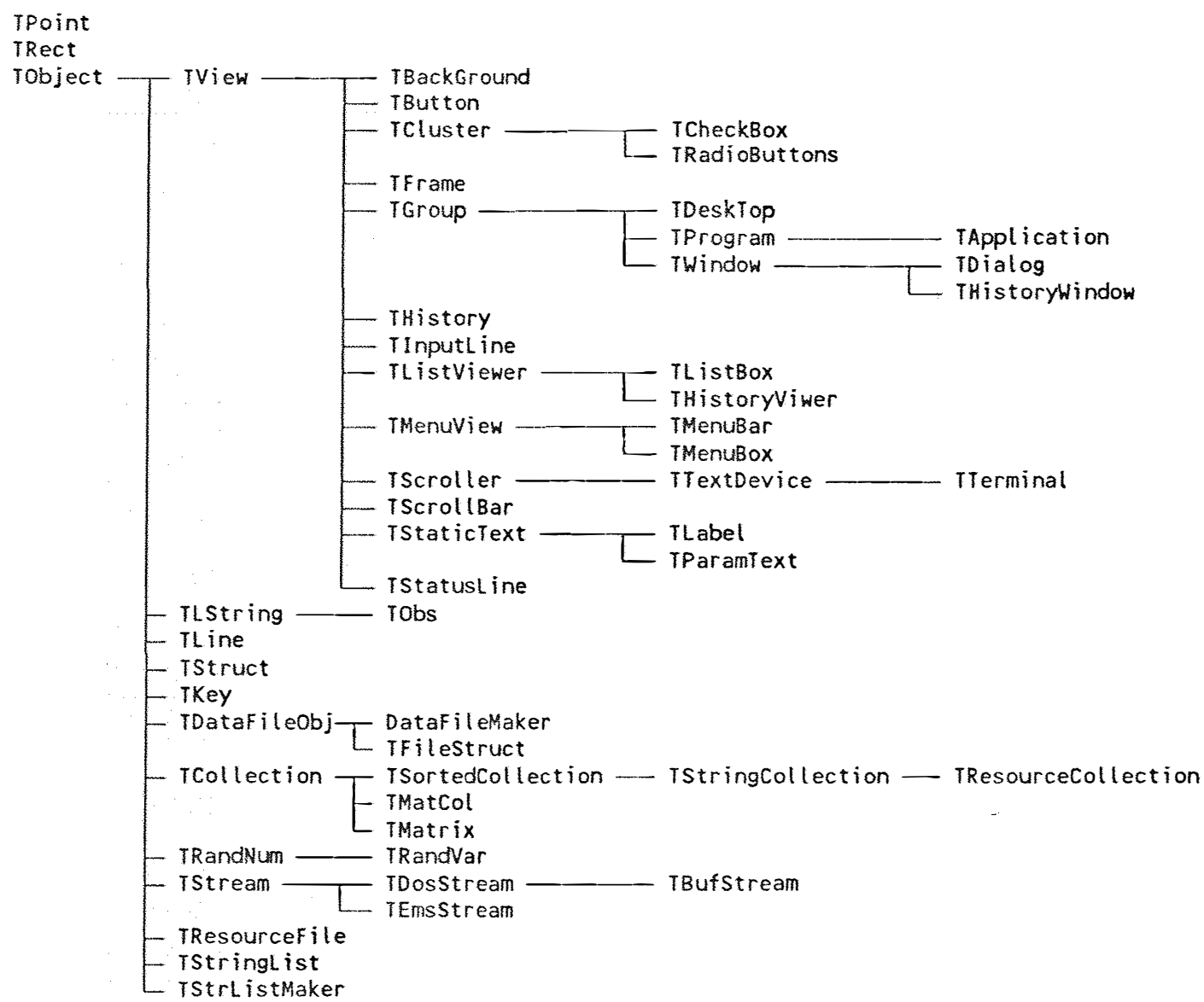

Figura 2. Árvore hierárquica após a inclusão dos novos objetos.

\subsubsection{Probabilidade}

BAux função

Declaração function $\operatorname{BAux}(x, p, q$, Inpf, Inqf, c: Float; upper: Boolean): Float ; 
Finalidade . BAux pode ser considerado um procedimento auxiliar que avalia a função beta incompleta $\mathrm{I}(\mathbf{p}, \mathbf{q} ; \mathbf{x})$. Retorna $\mathrm{I}(\mathbf{p}, \mathbf{q} ; \mathbf{x})$ se upper for False e 1 - I(p, q; x) se upper for True. Os parâmetros Inpf, Inqf e c devem conter, respectivamente, na entrada, $\operatorname{Ln}(\Gamma(\mathrm{p}+1)), \quad \operatorname{Ln}(\Gamma(\mathrm{q}+1))$ e $\operatorname{Ln}(\Gamma(p+q) /(\Gamma(p+1) \Gamma(p+1)))$. A implantação é uma adaptação do algoritmo proposto por MARDIA \& ZEMROCH (1978).

Beta função

SpecFunc

Declaração function Beta(z, w: Float): Float;

Finalidade Retorna o valor da função beta de parâmetros $\mathbf{z}$ e $\mathbf{w}$ através da sua relação com a função gama.

Betal função

SpecFunc

Declaração

function Betal (a, b, x: Float): Float;

Finalidade

Calcula o valor da função beta incompleta I $(\mathbf{a}, \mathbf{b} ; \mathbf{x})$. A implementação segue algoritmo proposto por PRESS et alli (1989).

BinProb função

SpecFunc

Declaração

function $\operatorname{BinProb}(n, k$ : Integer; $p$ : Float): Float;

Finalidade

Retorna o valor da probabilidade acumulada até $\mathbf{k}$ na distribuição binomial de parâmetros $\mathbf{n}$ e $\mathbf{p}$, tomando o valor $\mathrm{I}(\mathrm{k}, \mathrm{n}-\mathrm{k}+1 ; \mathrm{p})$.

Blnt função

SpecFunc

Declaração

function BInt (x, a, b: Float; upper: Boolean): Float;

Finalidade

Se upper for False o procedimento calcula a integral de probabilidade da distribuição beta, mais comumente conhecida como função beta incompleta, ou seja a integral entre 0 e x. Se upper for True então a área superior 1 I $(\mathbf{a}, \mathbf{b} ; \mathbf{x})$ será calculada, correspondendo aos limites de integração entre $\mathbf{x}$ e 1. A implementação é uma adaptação do algoritmo proposto por MARDIA 
\& ZEMROCH (1978).

BPerPt função

SpecFunc

Declaração

function BPerPt (p, a, b, eps: Float; upper: Boolean): Float;

Finalidade

Calcula os pontos percentuais da distribuição beta. Dado um valor de $\mathbf{p}, \mathbf{o}$ procedimento calcula o valor de $\mathrm{x}$ tal que $\mathrm{I}(\mathbf{a}, \mathbf{b} ; \mathbf{x})=\mathbf{p}$ se upper for False e I $(\mathbf{a}, \mathbf{b} ; \mathbf{x})=1$ - p para upper True. O algoritmo é o mesmo proposto por MARDIA \& ZEMROCH (1978).

CumPoisson função

SpecFunc

Declaração function CunPoisson( $k, x$ : Float): Float;

Finalidade Retorna $\mathrm{o}$ valor da probabilidade acumulada até $\mathrm{x}$ na distribuição de Poisson de parâmetro $\mathbf{k}$, calculada através da função gama incompleta, segundo indicação de PRESS et alli (1989).

FInt função

SpecFunc

Declaração

function FInt (x, v1, v2: Float; upper: Boolean): Float;

Finalidade

Calcula a integral de probabilidade da distribuição F com v1 e v2 graus de liberdade para o numerador e denominador, respectivamente, que poderão ser inteiros, fracionários ou infinitos, estes últimos indicados através de valores negativos para v1 e/ou para v2. Se upper é False ou True a área calculada será á esquerda ou à direita de x. A implementação segue MARDIA \& ZEMROCH (1978).

FPerPt função

SpecFunc

Declaração

function FPerPt(p, v1, v2, eps: Float; upper: Boolean): Float;

Finalidade

Calcula os pontos percentuais da distribuição F com v1 e v2 graus de liberdade, que poderão ser inteiros, fracionários ou infinitos. Se upper for False então o valor obtido será $x$ tal que $P(F \leq x)=p$ e $P(F \geq x)=p$ 
caso contrário. A indicação de graus de liberdade infinitos é feita por meio de valores de $\mathbf{v} \mathbf{1}$ e/ou de $\mathbf{v} \mathbf{2}$ negativos. Segue algoritmo proposto por MARDIA \& ZEMROCH (1978).

FProb função

SpecFunc

Declaração

function FProb(ni1, ni2, x: Float): Float;

Finalidade

Retorna o valor da função de distribuição de $F$ com ni1 e ni2 graus de liberdade no ponto $\mathbf{x}$, segundo modelo proposto por PRESS et al (1989).

GammLn função

SpecFunc

Declaração

funct ion GammLn(xx: Float): Float;

Finalidade

Retorna o valor de $\operatorname{Ln}(\Gamma(\mathbf{x} \mathbf{x}))$. A precisão é completa para $\mathbf{x x}>1$. Para 0 $<\mathbf{x x}<1$, a fórmula de reflexão deve ser utilizada primeiro. $\mathrm{O}$ algoritmo básico é dado por PRESS et al (1989).

GammP função SpecFunc

Declaração funct ion GammP(a, x: Float): Float;

Finalidade Retorna o valor da função gama incompleta de parâmetro a no ponto $\mathbf{x}$, conforme indicado por PRESS et al (1989).

GammQ função SpecFunc Declaração function $\operatorname{Garm} R(a, x$ : Float): Float;

Finalidade Retorna o valor do complemento da função gama incompleta ou 1 - GammP.

LogGamma função

SpecFunc

Declaração funct ion LogGanma(x: Float): Float;

Finalidade Retorna o valor $\operatorname{Ln}(\Gamma(\mathrm{x}))$ implementado a partir do algoritmo de MARDIA \& ZEMROCH (1978). 
Nlnt função

Declaração

Finalidade

Observações

NPerPt função

Declaração

Finalidade

Observações function NInt (x: Float; upper: Boolean): Float ;

SpecFunc Calcula a integral de probabilidade da distribuição normal de média zero e variância 1. Se upper for False a área calculada corresponde à cauda inferior, ou seja, calcula $\Phi(\mathbf{x})=\mathrm{P}(X \leq \mathbf{x})$. Se upper for True então a área calculada corresponde à $1-\Phi(\mathbf{x})=\mathrm{P}(\mathrm{X}>\mathbf{x})$, correspondente aos limites de $\mathbf{x}$ a $\infty$. Segue implementação de MARDIA \& ZEMROCH (1978).

Quando a média não for nula e/ou a variância não for 1 , o valor deve ser transformado antes de ser repassado a $\mathbf{x}$.

function NerPt (p, eps: Float; upper: Boolean): Float;

Calcula os pontos percentuais da distribuição normal com média 0 e variância 1, segundo implementação de MARDIA \& ZEMROCH (1978). Dado um valor de $p$, calcula $o$ valor de $x$ tal que $P(X \leq x)=p$ se upper for False ou tal que $P(X \geq x)=p$ se upper for True. $O$ parâmetro eps indica a precisão relativa desejada e é definido como parâmetro porque usualmente é definido como função do valor de $\mathbf{p}$ a ser considerado.

Se $X$ não tiver distribuição normal padrão, o valor obtido deve ser transformado para produzir o ponto percentual corretamente.

QuiSqr função

Declaração

function QuiSqr(ni, x: Float): Float;

Finalidade

Calcula o valor da função de distribuição no ponto $\mathbf{x}$ da distribuição quiquadrado com ni graus de liberdade. O cálculo é feito diretamente pela função gama incompleta, segundo PRESS et al (1989).

TInt função SpecFunc Declaração function TInt ( $x, n$ : Float; OneSided, upper: Boolean): Float; 
Finalidade $\quad$ o procedimento calcula a integral de probabilidade da distribuição $t$ com $\mathbf{n}$ graus de liberdade, onde $\mathbf{n}$ pode ser inteiro, fracionário ou infinito. Se OneSided for True, então será calculada a integral de probabilidade unilateral e se upper for False então a área calculada será $P(X \leq \mathbf{x})$, onde $X$ tem distribuição $t$ com $\mathbf{n}$ graus de liberdade e para upper True a área calculada será $\mathrm{P}(\mathrm{X} \geq \mathrm{x})=1-\mathrm{P}(\mathrm{X} \leq \mathrm{x})$. Se OneSided for False a área será dada pela integral bilateral, ou seja, $\mathrm{P}(|\mathrm{X}| \leq|\mathrm{x}|)$ correspondente à área entre $-x$ e $x$, e $P(|X| \geq|x|)=1-P(|X| \leq|x|)$ se upper for True. Se n for um valor negativo então o número de graus de liberdade será considerado como infinito. A implementação segue MARDIA \& ZEMROCH (1978).

TPerPt função

SpecFunc

Declaração

function TPerPt (p, n, eps: Float; upper, OneSided: Boolean): Float;

Finalidade $\quad O$ procedimento calcula, segundo algoritmo proposto por MARDIA \& ZEMROCH (1978), os pontos percentuais da distribuição $t$ com $\mathbf{n}$ graus de liberdade, onde $\mathbf{n}$ pode ser inteiro, fracionário ou infinito. Se OneSided for True, então são calculados niveis apropriados a um teste unilateral e se upper for False então os valores calculados serão tais que $\mathrm{P}(\mathrm{X} \leq \mathrm{x})=\mathbf{p}$, onde $\mathrm{X}$ tem distribuição $\mathrm{t}$ com $\mathbf{n}$ graus de liberdade e, para upper igual a True, os valores serão tais que $P(X \geq x)=p$. Se OneSided for False então serão calculados níveis apropriados para um teste bilateral, envolvendo basicamente as mesmas quantidades acima, apenas tomando o módulo de $\mathrm{X}$. Se $\mathbf{n}$ for um valor negativo então o número de graus de liberdade será considerado como infinito.

TProb função

SpecFunc

Declaração function TProb(ni, x: Float): Float;

Finalidade Retorna o valor da função de distribuição da distribuição $t$ de Student com 
ni graus de liberdade no ponto $\mathrm{x}$, calculada da maneira proposta por PRESS et al (1989).

$X 2 A u x$ função

SpecFunc

Declaração

function X2Aux (x, v, vfac: Float; upper: Boolean): Float;

Finalidade

O procedimento avalia a função gama incompleta. Se upper for True os limites de integração estarão entre $\mathrm{x}$ e $\infty$ e se upper for False, os limites estarão entre $0 \mathrm{e} \mathbf{x}$. Os parâmetros $\mathbf{v}$ e vfac são respectivamente, o parâmetro da gama incompleta e $\operatorname{Ln}(\Gamma(\mathrm{v}))$, tal como proposto por MARDIA \& ZEMROCH (1978).

X2Int função

SpecFunc

Declaração

function $X 2 \operatorname{lnt}(x, v$ : Float; upper: Boolean): Float;

Finalidade

X2Int calcula a integral de probabilidade da distribuição qui-quadrado com $\mathbf{v}$ graus de liberdade onde $\mathbf{v}$ pode ser inteiro ou fracionário. Se upper é False então será calculada a área à esquerda de $\mathbf{x}$ ou $\mathrm{P}(\mathrm{X} \leq \mathrm{x})$, enquanto que se upper for True a área será à direita de $\mathbf{x}$ ou $\mathrm{P}(\mathrm{X} \geq \mathbf{x})=1-\mathrm{P}(\mathrm{X}$ $\leq \mathbf{x})$, que corresponde aos limites de integração de $\mathbf{x}$ a $\infty$. A implementação é a mesma de MARDIA \& ZEMROCH (1978).

$X 2 P e r P t$ função

SpecFunc

Declaração

funct ion $\times 2 P e r P t(p, v$, eps: Float; upper: Boolean): Float;

Finalidade Calcula, tal como propõe MARDIA \& ZEMROCH (1978), os pontos percentuais da distribuição qui-quadrado com $\mathbf{v}$ graus de liberdade onde $\mathbf{v}$ pode ser inteiro ou fracionário. Dado um valor de $\mathbf{p}$, o procedimento calcula o valor $\mathrm{x}$ tal que $\mathrm{P}(\mathrm{X} \leq \mathrm{x})=\mathrm{p}$ se upper for False, ou tal que $\mathrm{P}(\mathrm{X} \geq \mathrm{x})$ $=\mathrm{p}$ se upper for True. 


\subsubsection{Avaliação de expressões}

Eval função

EvalExpr

Declaração funct ion Eval (xx: PFArray; formula: string; var NumErr: (nteger): Float;

Finalidade Dada uma expressão matemática transmitida através do parâmetro formula e um ponteiro para um vetor de números reais passado para $\mathbf{x x}$, Eval retorna o valor da expressão para aquele conjunto de valores apontados por $\mathbf{x x}$. A implementação de Eval segue de muito perto a forma proposta por HOROWITZ \& SAHNI (1984) e consiste numa adaptação da implementação proposta por AZEVEDO FILHO (1988).

Uma expressão matemática a ser avaliada por Eval contém identificadores de variáveis, de funções matemáticas e operadores aritméticos e boleanos. As regras para construção da expressão serão descritas na Tabela 10.

Tabela 10. Operadores aritméticos previstos em Eval.

Operador Finalidade

\begin{tabular}{cl}
\hline+ & Soma \\
- & Subtração \\
$*$ & Produto \\
$/$ & Divisão \\
$\wedge$ & Potenciação \\
\hline
\end{tabular}

trabalhados dentro da ordem de prioridade ${ }^{*},{ }^{*}, /,+,-$. Nos casos de operadores com mesmo nível de prioridade (produto e divisão, soma e subtração) a prioridade é de quem vem primeiro, da esquerda para a direita.

A Tabela 11 apresenta os operadores utilizados. 
Tabela 11. Operadores lógicos ou boleanos aceitos por Eval.

\begin{tabular}{lll} 
Símbolo & Descrição & Após padronização \\
\hline$>$ & maior & $>$ \\
$>=$ & maior ou igual & $\mathrm{G}$ \\
$<=$ & menor ou igual & $\mathrm{L}$ \\
$=$ & igual & $=$ \\
$<>$ & diferente & $\mathrm{D}$ \\
$\&$ & e (and); & $\&$ \\
L & ou (or); & I \\
\hline
\end{tabular}

Toda expressão boleana retorna o valor 0 ou 1 conforme seja falsa ou verdadeira. A alteração de ordem para a avaliação dessas expressões deve ser construída através do uso de parênteses, uma vez que todos os operadores boleanos possuem a mesma prioridade. A Tabela 12 mostra as funções matemáticas disponíveis.

Tabela 12. Funções matemáticas disponíveis em Eval.

\begin{tabular}{lll} 
Símbolo & Descrição & Após padronização \\
\hline LN & Logaritmo natural & F00 \\
LOG & Logaritmo decimal & F01 \\
ABS & Valor absoluto & F02 \\
ASN & Arco seno & F03 \\
ACS & Arco cosseno & F04 \\
SEN & Seno & F05 \\
COS & Cosseno & F06 \\
ATN & Arco tangente & F07 \\
TAN & Tangente & F08 \\
EXP & Exponencial & F09 \\
$!$ & Negação boleana & F10 \\
NEG & Negativo & F11
\end{tabular}


A função ! é a negação boleana. Assim, se o valor de uma expressão lógica é 1 (verdadeira), essa função devolve o valor 0 (falsa) e vice-versa. A função NEG deve ser utilizada sempre que houver necessidade do operador unário , que não está definido como operador.

A ordem de prioridade para execução de uma expressão pode ser alterada através da utilização de parênteses, que torna toda expressão em seu interior prioritária para avaliação. Dentro dos parênteses a prioridade retorna como definida anteriormente. Do mesmo modo, todo argumento para as funções matemáticas deverá ser indicado através do uso de parênteses.

NumErr retorna uma possível condição de erro ocorrido durante o cálculo, cujo significado é dado na Tabela 13.

Tabela 13. Códigos de erro reconhecidos por Eval.

NumErr Descrição

\begin{tabular}{ll}
\hline 0 & Avaliação normal \\
-1 & Divisão por zero. \\
-2 & Argumento ilegal para logaritmo. \\
-3 & Argumento ilegal para função trigonométrica \\
\hline
\end{tabular}

Para que Eval possa ser chamada com sucesso para a avaliação da expressão, ela já deverá ter sido tratada pelos quatro procedimentos StdForm, StdFunc, FindGenError e PostFix.

FactEval função

EvalExpr

Declaração function FactEval (xx: PMArray; formula: string): Integer;

Finalidade FactEval objetiva retornar um valor inteiro resultante da avaliação de formula tomando como base os valores inteiros contidos em $\mathbf{x x}^{\wedge}$. Sua finalidade básica é avaliar condições boleanas (embora o valor resultante não seja somente 0 ou 1, como em StrEval) em chaves numéricas do tipo inteiro, 
definidas em TKey. Não reconhece nenhuma função, apenas os operadores aritméticos e os boleanos. A expressão que será passada ao parâmetro formula deve ser tratada anteriormente por StdForm. O tratamento de erros é feito por FindCondError, com as ressalvas já discutidas.

Find CondError procedimento

EvalExpr

Declaração procedure FindCondError(var NumErr, i : Integer; st: String);

Finalidade Finalidade semelhante à FindGenError, agora destinada a varrer a expressão st que será posteriormente avaliada por StrEval ou por FactEval. Nas situações onde se aplicam, os códigos de erros descritos para FindGenError são os mesmos que retornam através de NumErr. Note-se que se for utilizado algum operador ilegal para StrEval mas legal para FactEval (operadores -, / e *) nenhuma mensagem de erro retornará de FindCondError. Através de i retorna o índice onde foi detectado o erro, estando o erro propriamente dito desse ponto para trás. Para st deve ser passada a expressão a ser avaliada, logo após ter sido tratada por StdForm.

FindGenError procedimento EvalExpr

Declaração procedure FindGenError (var NerEer , i Integer; fomula: string);

Finalidade FindGenError, adaptada a partir de uma rotina proposta por AZEVEDO FILHO (1988), tem por objetivo varrer a expressão passada através do parâmetro formula a procura de erros de definição. Deve ser utilizado sempre que a expressão se refira à números (reais ou inteiros). Em NumErr retorna o código de erro e em $\mathbf{i}$ retorna o índice do caractere onde foi detectado o erro. Os números que correspondem à condição de erro são dados na Tabela 14. 
Tabela 14. Condições de erro reconhecidos na especificação da expressão a ser avaliada.

\begin{tabular}{cl} 
NumErr & Descrição \\
\hline 0 & Nenhum erro \\
-1 & Caractere ilegal no início da fórmula \\
-2 & Caractere ilegal após X \\
-3 & Final de fórmula inesperado \\
-4 & Caractere ilegal após ( \\
-5 & Caractere ilegal após ) \\
-6 & Problemas nos parênteses \\
-7 & Problemas nas aspas \\
-8 & Caractere ilegal após função \\
-9 & Caractere ilegal após operador \\
-10 & Dois pontos no número \\
-11 & Caractere ilegal após número \\
-12 & Caractere ilegal \\
\hline
\end{tabular}

Através do valor que retorna em i, tem-se a posição em que FindGenError encontrou um erro. Note-se que não é necessariamente nessa posição que o erro se encontra. Esse índice deve ser considerado como um índice tal que o erro se encontra nele ou antes dele.

PostFix função

function PostFix(fomula: string): string;

Declaração PostFix toma como parâmetro formula a expressão já padronizada e Finalidade depurada de erros e a transforma da notação infixa para a notação pós-fixa, para posterior avaliação. PostFix segue, nas suas linhas básicas, o algoritmo proposto por HOROWITZ \& SAHNI (1984). Os detalhes da implementação segue os de AZEVEO FILHO (1988). 
StdForm procedimento

EvalExpr

Declaração procedure StdForm(var fomula: string):

Finalidade Da expressão repassada para formula, elimina todos os espaços em branco, torna todas as letras maiúsculas e padroniza as variáveis (para que todas passem a ter o mesmo comprimento) e os operadores que utilizam mais de um caractere. Assim, o rótulo $\mathrm{x} 1$, que deve ser necessariamente utilizado para indicar a primeira posição do vetor (ou a primeira variável), será transformado em X0001, x10 em X0010 e assim por diante. Observe-se, novamente, que o índice $x 1$ indicará que $o$ valor dessa variável encontra-se na posição 1 de $x^{\wedge}$ (parâmetro de Eval), $x 10$ na posição 10 , e assim por diante. A amplitude de variáveis, portanto, vai de X0001 a X9999. Tal implementação segue a de AZEVEDO FILHO (1988).

StdFunc procedimento

EvalExpr

Declaração

procedure StdFunc(var formula: string);

Finalidade

$\mathrm{Na}$ expressão passada através de formula, padroniza o modo como as funções serão processadas pelas demais rotinas de tratamento de expressões. A descrição de Eval mostra o modo como as funções são padronizadas. A sua chamada não precisa necessariamente preceder StdForm, mas ambas devem preceder FindGenError. Esse tratamento é o mesmo proposto por AZEVEDO FILHO (1988).

StrEval função

EvalExpr

Declaração

function StrEval(YY: PStrArray; fomula: string): Integer;

Finalidade

O procedimento StrEval toma como parâmetro um ponteiro para um vetor de ponteiros (YY) para cadeias e uma expressão lógica (formula) que estabelece a relação existente entre essas cadeias. O retorno de StrEval é um inteiro igual a 0 (a expressão é falsa) ou a 1 (a expressão é verdadeira). O uso básico dessa função neste contexto é estabelecer quais registros deverão ser acessados, ou seja, estabelecer um sistema de acesso aos registros através 
de chaves alfanuméricas definidas no objeto TKey, avaliando cada conjunto de chaves através de StrEval e decidindo quais serão escolhidos.

Evidentemente nenhuma das funções e muitos operadores não se aplicam à avaliação de expressões com esse tipo de variável. Assim, os únicos operadores que permanecem é o operador aritmético + e os operadores lógicos. Toda cadeia entre aspas é considerada como constante por StrEval.

\subsubsection{Matrizes}

NullMat função

Matrix

Declaração

function Nul lingt (mt: Char; nr, nc: Word): FMat rix;

Finalidade NullMat retorna um ponteiro para uma matriz nula do tipo $\mathbf{m t}$ com $\mathbf{n r}$ linhas e nc colunas. Esse procedimento é muito útil quando se deseja definir uma matriz já pronta para ser preenchida com valores.

ReadMat função

Declaração

function Readkt (var TFile: Text): PMatrix;

Finalidade

ReadMat lê e constrói uma matriz a partir do arquivo texto TFile e retorna um ponteiro para essa matriz. $\mathrm{O}$ arquivo texto que contém a matriz deve possuir as seguintes características:

- Na primeira linha deverão ser indicados o número de linhas, de colunas e o tipo da matriz, separados por um delimitador de Delim. Os tipos devem ser indicados como $G$ para matriz geral, $S$ para matriz simétrica, D para matriz diagonal e $T$ para matriz triangular inferior.

- A partir da segunda linha deverão vir os elementos de cada linha da matriz separados por pelo menos um espaço. Um ENTER indicará o final da linha e o início da próxima, se for o caso.

- A palavra FIM deve ser utilizada na linha seguinte à última para indicar 
que a matriz já foi lida.

Observações Como se pode notar, o procedimento que chama ReadMat deve antes abrir o arquivo texto e, após a leitura ter sido feita, se encarregar de fechá-lo. Uma vez que o final da leitura se dá através da palavra FIM, é possível ler uma matriz de um arquivo texto com outras linhas desde que a matriz lida seja a primeira.

\subsubsection{Outras rotinas}

Factln função

SpecFunc

Declaração

function FactLn(n: Integer; var F: PFArray): Float;

Finalidade

Tem finalidade semelhante à Factrl com a diferença que devolve Ln(n!), todos os cálculos são feitos utilizando a função Gama e a dimensão prevista para o vetor $\mathbf{F}^{\wedge}$ é de 100 componentes. Para $\mathbf{n}>99$ o cálculo é feito diretamente e não é armazenado na tabela.

Observações $\quad \mathbf{O}$ vetor $\mathbf{F}$ deve ser dimensionado antes da primeira chamada e inicializado com valores negativos $(-1.0$, por exemplo)

GL procedimento

GLib

Declaração

Finalidade

procedure G(var W: ANArray; $k, n$ : Word) ;

Este procedimento é utilizado para construir blocos regulares de valores inteiros e atribuí-los às posições de $\mathbf{W}$. Assim, os $\mathbf{n}$ primeiros valores de $\mathbf{W}^{\wedge}$ serão iguais a 1 , os $\mathbf{n}$ seguintes serão iguais a 2 , e assim por diante. Este processo continua até que o bloco com o valor $\mathbf{k}$ seja construído. A partir desse momento, o processo se repete, com os próximos $\mathbf{n}$ valores sendo iguais a 1 , etc., até que todos os valores do vetor $\mathbf{W}^{\wedge}$ tenham sido atribuídos. O algoritmo utilizado é o proposto por BAKER (1986).

Observação $\quad$ O vetor apontado por W deverá ser dimensionado antes da chamada de GL 
e a quantidade de posições após a posição 0 deverá ser colocado em $\mathbf{W}^{\wedge}[0]$.

\subsection{Considerações Finais}

O Turbo Vision, biblioteca implementada juntamente com a versão 6.0 do Turbo Pascal, revelou-se um excelente ambiente de desenvolvimento para processamento de rotinas científicas, uma vez que todos os objetos desenvolvidos o foram como extensão dos objetos padrões do Turbo Vision. Além disso, ao fornecer suporte para uma interface de alta qualidade e para a programação orientada a eventos, ele proporciona um ambiente agradável e padronizado para o usuário.

O conjunto de objetos desenvolvido para o manuseio de arquivos de dados exemplifica o processo de construção de uma aplicação Turbo Vision no que diz respeito à utilização da interface. O outro objetivo, foi construir um aplicativo para gerenciamento de arquivos de dados que fosse ao mesmo tempo totalmente interativo, fácil de utilizar e aprender e razoavelmente flexível no tratamento dos dados. A intenção aqui não foi construir algo para uma extensão posterior, embora sob alguns aspectos isso possa ser feito.

Um objeto que exemplifica muito bem a potencialidade do conjunto básico de objetos Turbo Vision é TMatrix. Ele é um descendente do objeto padrão TCollection e sabe dimensionar e redimensionar o seu espaço na memória, sabe como eliminar-se da memória, como ser guardado e recuperado de um arquivo (ou, mais geralmente, de um stream), como ser impresso através de um dispositivo de saída e, além disso, sabe, através dos métodos específicos implementados, como realizar as mais diferentes e úteis operaçōes matriciais. $\mathrm{O}$ objeto TMatCol, também um descendente de TCollection, tem como finalidade manter uma coleção de objetos TMatrix, o que fornece bem a potencialidade de utilização tanto desse objeto como de seu ancestral TCollection.

O objeto TRandNum e seu descendente TRandVar implementam a geração de variáveis aleatórias de distribuição uniforme e de outras distribuiçōes, respectivamente. Através do polimorfismo e da hereditariedade, por exemplo, o gerador de uniformes utilizado 
em TRandVar pode ser trocado sem que nada necessite ser alterado a não ser a substituição de um método.

Os objetos, exceto os de manuseio de arquivos, têm ampla possibilidade de expansão. Descendentes de TMatrix poderão ser obtidos de modo a que sejam implementadas outros métodos, aumentando a sua funcionalidade e aproveitando toda a estrutura já desenvolvida. Um objeto descendente de TMatCol, por exemplo, poderia ser obtido para a implementação de um interpretador de fórmulas envolvendo matrizes ao invés de números. A adequação das rotinas de avaliação de expressões para esse fim não é difícil. Novos descendentes de TRandVar poderão ser obtidos para a inclusão de geradores de variáveis aleatórias de distribuições. Combinando os geradores já disponíveis e o objeto TMatrix, facilmente se constrói um gerador para a disiribuição multinormal e para a distribuição de Wishart.

Todos os objetos construídos poderão ser utilizados também como "caixa preta", ou seja, simplesmente colocados a funcionar sem que seja necessário qualquer conhecimento da sua estrutura interna.

Além dos objetos mencionados, outras rotinas isoladas de grande utilidade foram implementadas. A rotina de avaliação de expressões possibilita avaliar qualquer expressão matemática, desde que os operadores e as funções sejam as pré-definidas. Além disso, esse algoritmo foi modificado para possibilitar o acesso condicional a registros através de chaves numéricas ou de caracteres. Outro conjunto muito útil, em particular para a estatística, é composto pelas rotinas que obtêm valores de funções importantes tais como a gama e a beta e por aquelas que efetuam o cálculo de probabilidades e dos pontos percentuais das distribuiçōes mais comuns com uma precisão muito boa. Essas últimas, quando for o caso, aceitam graus de liberdade fracionários e infinitos e possibilitam escolher se a área desejada está localizada à esquerda ou à direita. Na distribuição $t$, ainda existe a possibilidade de indicar se a obtenção se refere à tabela uni ou bilateral.

Uma possível desvantagem dessa biblioteca é a sua ligação muito íntima com o ambiente específico Turbo Pascal e, por conseqüência, pelo menos neste momento, com o equipamento IBM-PC ou compatível e com o sistema operacional MS-DOS ou compatível. Em contrapartida, entretanto, deve-se ressaltar que o Turbo Pascal é um ambiente de ampla 
utilização e de boa facilidade de aprendizado, sendo perfeitamente possível, portanto, imaginarse que essa linguagem forneceria um bom suporte para disciplinas de estatística computacional e matemática numérica, nas quais essa biblioteca poderia ser utilizada. Além do mais, deve-se levar em conta que uma biblioteca desse tipo se aproveita da hierarquia já pré-definida baseada na larga experiência que possuem os desenvolvedores do Turbo Pascal. Com ela, torna-se possível construir programas de aspecto profissional através de um investimento de tempo relativamente pequeno. Também não se pode desprezar o atual estágio de evolução do Pascal, onde a tendência é a construção de ambientes de desenvolvimento onde os códigos construídos são cada vez mais fáceis de ser traduzidos, pela própria concordância com as bibliotecas de apoio, e até compartilhados para uso com programas construídos na linguagem $\mathrm{C}++$. 


\section{REFERÊNCIAS BIBLIOGRÁFICAS}

AZEVEDO FILHO, A. J. B. V. Análise econômica de projetos: "Software" para situações determinístas e de risco envolvendo simulação. Piracicaba, 1988. 127 p. (Mestrado ESALQ/USP)

BAKER, R. J. GLIM 3.77 Reference guide. In: Payne, C. D. ed. The GLIM system release 3.77. London, Numerical Algorithms Group, 1986. 80 p.

BEATON, A. E. The use of special matrix operators in statistical calculus. Harvard, 1964 (Ed.D. thesis - Harvard University).

BJÖRCK, A. Solving least squares problems with Gramm-Schimidt orthogonalization. BIT, 7: 1-21, 1967.

BORLAND. Turbo Pascal Programer's Guide, version 6.0. Borland International Inc., Scotts Valley, 1990. $370 \mathrm{p}$.

BORLAND. Turbo Pascal Turbo Vision Guide, version 6.0. Borland International Inc., Scotts Valley, 1990. $370 \mathrm{p}$.

BORLAND. Turbo Pascal User's Guide, version 6.0. Borland International Inc., Scotts Valley, 1990. $259 \mathrm{p}$. 
BUSTOS, O. H. \& ORGAMBIDE, A. C. F. Simulação estocástica. Teoria e algoritmos. Rio de Janeiro, $10^{\circ}$ Simpósio Nacional de Probabilidade e Estatística, 1992. 152 p.

CHAMBERS, J. M. Computacional methods for data analysis. New York, John Wiley \& Sons, 1977. 268 p.

COOK, D., CRAVEN; A. H.; CLARKE, G. M. Statistical computing in Pascal. London, Edward Arnold Publishers, 1985. 171 p.

DACHS, J. N. W. Estatística computacional. Uma introdução em Turbo Pascal. Rio de Janeiro, Livros Técnicos e Científicos Editora Ltda, 1988. 236 p.

EZZEL, B. Object oriented programming in Turbo Pascal 5.5. Reading, Addison-Wesley Publishing Company, 1989. 375 p.

FORSYTHE, G. E.; MALCOM, M. A.; MOLER, C. B. Computer methods for mathematical computations. Englewood Cliffs, Prentice-Hall, Inc., 1977. 259 p.

GOLUB, G. H. \& REINSCH, C. Singular value decomposition and least square solutions. In: Wilkinson, J. H. \& Reinsch, C. ed. Handbook for automatic computation. Linear algebra. Berlim, Springer-Verlag, 1971. v.1, p. 134-151.

GOODNIGHT, J. H. A tutorial on the SWEEP operator. The American Statistician, 33: 149-158, 1979.

HOROWITZ, E. \& SAHNI, S. Fundamentos de estruturas de dados. Rio de Janeiro, Editora Campus Ltda, 1984. 494 p.

KENNEDY, W. J. \& GENTLE, J. E. Statistical computing. New York, Marcel Dekker, Inc., 1980. 591 p. 
KNUTH, D. E. The art of computer programming 2: Seminumerical algorithms, 2nd. ed. Reading, Addison-Wesley Publishing Co., 1981. p.

LAWSON, C. L. \& HANSON, R. J. Solving least squares problems. Englewood Cliffs, Prentice-Hall, Inc., 1974. 340 p.

LEON, S. J. Linear algebra with applications. New York, Macmillan Publishing Co., Inc., 1980. $338 \mathrm{p}$.

MARDIA, K. V. \& ZEMROCH, P. J. Tables of the $\mathbf{F}$ and related distributions with algorithims. London, Academic Press, 1978. 256 p.

MCCULlAGH, P. \& NELDER, J. A. Generalized linear models, 2nd. ed. London, Chapman and Hall, 1989. 511 p.

MOOD, A. M.; GRA YBILL, F. A.; BOES, D. C. Introduction to the theory of statistics, 3rd. ed. Singapore, McGraw-Hill Book Company, 1974. 564 p.

O'BRIEN, S. K. Turbo Pascal advanced programmer's guide. Berkeley, Borland/ Osborne/McGraw-Hill, 1988. 463 p.

O'BRIEN, S. K. Turbo Pascal 6. The complete reference. Berkeley, Borland-Osborne/McGraw-Hill, 1991. 690 p.

PRESS, W. H.; FlANNERY, B. P.; TEUKOLSKY, S. A.; VETTERLiNG, W. T. Numerical recipes in Pascal. The art of scientific computing. Cambridge, Cambridge University Press, 1989. 759 p.

THISTED, R. A. Elements of statistical computing. Numerical computation. New York, Chapman and Hall, 1988. 427 p. 
VETTERlinG, W. T.; TEUKOLSKY, S. A.; PRESS, W. H.; FLANNERY, B. P. Numerical recipes example book (Pascal). Revised edition. Cambridge, Cambridge University Press, 1989. 223 p. 


\section{Apêndice 1}

Este apêndice contém a referência de objetos, procedimentos e funções, tipos, variáveis e constantes não listados na seção 4 de Resultados. Estão organizados em ordem alfabética, para maior facilidade de localização. A forma de apresentação segue o mesmo padrão utilizado naquela seção.

\section{Referência dos Demais Objetos}

\section{TBrowserDialog}

CrtData

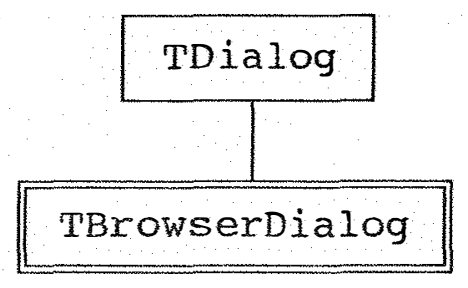

TBrowserDialog é um objeto que visa fornecer um quadro de diálogo para a edição de registros. Edita um registro de cada vez e se não há possibilidade de editar todos os campos na mesma tela, trabalha com grupos de até 8 campos por vez.

Métodos

Init

Declaração

constructor Init MexLabSize, Num: Byte; Rec: Word; Alab: PStringArray; ASize: PByteArray); 
Finalidade Constrói uma caixa de diálogo que conterá Num campos com a finalidade de editar o registro de número Rec. Alab é um ponteiro para um vetor de cadeias que contém os rótulos dos campos cujas dimensões estão no vetor apontado por ASize. MaxLabSize é a maior dimensão de campo daqueles que estarão na caixa de diálogo.

\section{HandleEvent}

Declaração procedure HandleEvent (var Event: TEvent); virtual;

Finalidade Trata os eventos não tratados por TDialog. As constantes não padronizadas associadas aos eventos estão na Tabela 15.

Tabela 15. Eventos tratados por TBrowserDialog.HandleEvent Eventos Descrição

Comandos

cmSave

Grava a coleção de registros tal como ela se encontra no momento.

Teclas

kbPgDn Pula RecStep registros para baixo.

kbDown Pula 1 registro para baixo.

kbPgUp Pula RecStep registros para cima.

kbUp Pula 1 registro para cima.

kbCtrlHome Retorna ao primeiro registro da coleção.

kbCtrlEnd Retorna ao último registro da coleção.

kbCtrlLeft Lista 8 campos à esquerda do primeiro listado na caixa.

kbCtrlRight Lista os 8 campos à direita do último listado. 


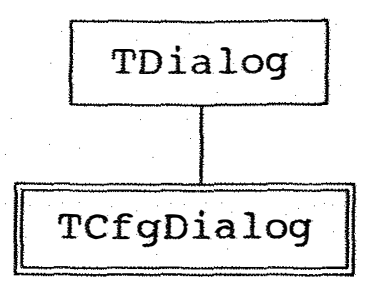

TCfgDialog é uma caixa de diálogo cuja finalidade é definir diretórios para acesso à dados, texto, chaves, etc., definir dispositivos de entrada, etc. Essa configuração pode ser armazenada em disco ou simplesmente mudada sem que o conteúdo do arquivo seja alterado.

\section{Métodos}

Init

Declaração

constructor Init;

Finalidade

Inicializa a caixa de diálogo, constrói e insere todos os demais objetos dentro da caixa tais como linhas de entrada, botões, caixas de verificação, etc.

HandleEvent

Declaração procedure Hand leEvent (var Event: TEvent); virtual ;

Finalidade A caixa de diálogo TCfgDialog gera vários eventos de comando estranhos à TDialog que são tratados por HandleEvent. Os comandos tratados são os usuais de ajuda, gravação, etc.

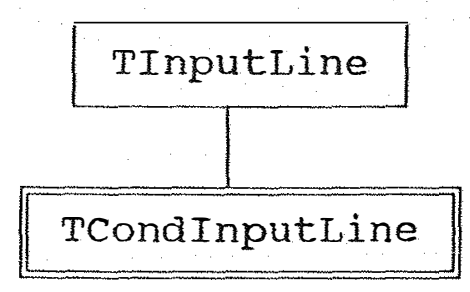

TCondInputLine tem por objetivo estabelecer uma linha para entrada de uma 
fórmula. Possui procedimentos para converter a fórmula numa forma apropriada e para detecção e impressão de erros, se for o caso.

\section{Métodos}

Error-

Declaração

procedure Error (Code: Integer);

Finalidade

Chamada por Valid caso a fórmula constante na linha de entrada tenha algum erro detectado. O parâmetro Code identifica um tipo de erro, valor este que retorna do procedimento FindCondError chamado por Valid. A descrição de TFileStruct.CondToInt e dos procedimentos de avaliação de fórmulas da unit EvalExpr fornece maiores detalhes sobre a forma de especificação das fórmulas.

\section{Valid}

Declaração

function Valid(Command: Word): Boolean; virtual;

Finalidade Valid chama inicialmente StdForm e a seguir FindCondError. Se o parâmetro NumErr de FindCondError retornar um valor diferente de zero Valid retorna False e aciona Error. Se NumErr retorna o valor zero, a linha de entrada é fechada com sucesso e a expressão modificada por StdForm retorna do objeto.

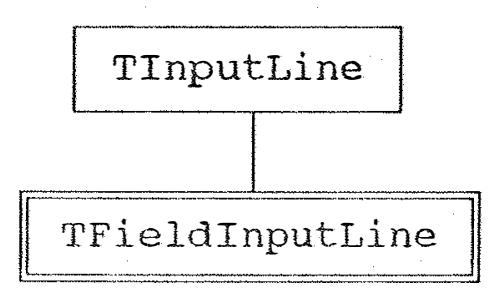

TFieldInputLine é um descendente de TInputLine cuja finalidade é oferecer uma linha de entrada apropriada para a indicação de campos de registros que deverão sofrer alguma ação, como listagem, eliminação, etc.. Veja a 
descrição do método TFileStruct.TokenToInt para maiores detalhes acerca da especificação da linha de entrada.

\section{Métodos}

Error -

Declaração

procedure Error(Num: Integer);

Finalidade

Listar possíveis erros na especificação da linha de entrada. Os erros previstos estão na Tabela 16.

Tabela 16. Códigos de erros reconhecidos por TFieldInputLine

Num

Descrição

$-1$

Linha de entrada não pode ser vazia

$-2$

Primeiro caractere não pode ser -

$-3$

Caractere inválido na definição de campo

Valid

Declaração

function Valid(Comrand: Word): Boolean; virtual;

Finalidade

Valid retorna False se alguma condição de erro ocorre na especificação dos campos a serem tratados. A linha somente pode ser abandonada após a correção dos erros.

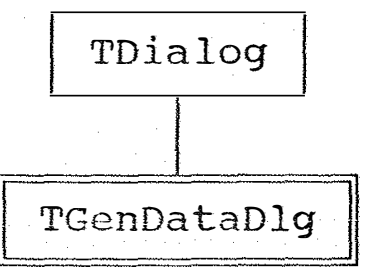

TGenDataDlg objetiva fundamentalmente fornecer uma caixa de diálogo para 
que possam ser indicadas as fórmulas para geração de novas colunas no arquivo de interesse. Essa geração é feita de modo incondicional.

Campos

AFile

Declaração AFile: PDataFileObj;

Finalidade Ponteiro para o objeto relacionado ao arquivo de dados ao qual se deseja adicionar as colunas que serão resultantes de uma combinação das colunas já existentes expressa através de uma fórmula matemática.

ListField, ListOper

Declaração ListField: PCollection;

ListOper: PCollection;

Finalidade Ponteiros para as coleções que irão armazenar os rótulos relativos aos campos e aos operadores e funções para que possam ser exibidos por uma caixa de lista.

$A B o x$

Declaração ABox: PListBox;

Finalidade Ponteiro para uma caixa de lista cuja finalidade é exibir as listas apontadas por ListField e ListOper.

ALabel

Declaração Alabel: Plabel:

Finalidade Aponta para um rótulo que irá identificar qual lista estará sendo exibida no momento.

FormLine

Declaração FormLine: PView;

Finalidade Aponta para a linha de entrada onde deverão ser introduzidas as fórmulas 
para a geração das novas colunas.

\section{WhatList}

Declaração WhatList: ListType;

Finalidade Indica a lista que no momento está em exibição.

Métodos

Init

Declaração

const ructor Init (DatafName: PathSt $r$ );

Finalidade

Inicializa o objeto e constrói a caixa de diálogo com os demais objetos internos à ele. Inicializa o objeto apontado pelo campo AFile abrindo o arquivo DataFName. Preenche as coleçōes apontadas por FieldList e por OperList.

Done

Declaração

destructor Done; virtual;

Finalidade

Libera a memória reservada para os campos do objeto.

HandleEvent

Declaração

procedure HandleEvent (var Event: TEvent); virtual;

Finalidade Trata os eventos complementares à TDialog. De modo semelhante aos anteriores, trata basicamente da mudança de coleções para a caixa de lista.

Tabela 17. Eventos tratados por TGenDataDlg. HandleEvent

Eventos Descrição

Comandos

cmKeyListBox Comando para que a caixa de lista passe a exibir as chaves.

cmOperListBox Caixa de lista deve passar a exibir os operadores.

cmFieldListBex Caixa de lista deve passar a exibir os rótulos de campos. 


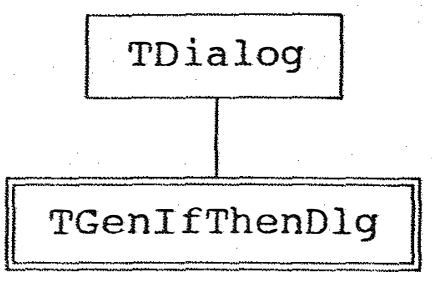

TGenIfThenDlg tem por objetivo fornecer uma caixa de diálogo onde estão presentes três linhas de entrada: uma para o estabelecimento de uma condição lógica, outra para indicação de uma fórmula caso a condição seja verdadeira e outra para uma fórmula que será utilizada caso a condição seja falsa. Para maiores detalhes veja a descrição de TFileStruct.GenIfThen.

Campos AFile

Declaração AFile: PFilestruct;

Finalidade Ponteiro para um objeto associado a um arquivo de dados e a um de chaves.

\section{ListField, ListKey, ListOper}

Declaração ListField: PCollection;

ListKey: PCollection;

ListOper: PCollection;

Finalidade Ponteiros para coleções de rótulos para exibição na caixa de lista interna à caixa de diálogo, respectivamente, rotulando os campos, as chaves e os operadores e funções disponíveis.

$A B o x$

Declaração

ABox: PListBox;

Finalidade Ponteiro para a caixa que lista as coleções de que trata o ítem anterior. 


\section{ALabel}

Declaração

Alabel: PLabel;

Finalidade

Ponteiro para o rótulo que identifica a lista que no momento está sendo exibida pela caixa de lista.

lfField, ThenForm, ElseForm

Declaração

IfField: PVien;

ThenForm: PView;

ElseForm: PVien;

Finalidade Ponteiros para linhas de entrada, respectivamente para a condição imposta às chaves e as fórmulas que serão aplicadas aos registros caso a condição seja verdadeira ou falsa.

\section{HaveKey}

Declaração HaveKey: Boolean;

Finalidade Campo lógico que indica a presença de chaves.

WhatList

Declaração WhatList: ListType;

Finalidade Indica qual lista está sendo exibida no momento na caixa de lista.

\section{Métodos}

Init

Declaração

constructor Init (DatafName, KeyfNarre: PathStr);

Finalidade

Constrói a caixa de diálogo, a caixa de lista e as linhas de entrada. Todas as informações externas são trazidas dos arquivos de dados DataFName e de chaves KeyFName. Se KeyFName for vazio atribuirá False a HaveKey.

Done

Declaração

destructor Done; virtual; 
Finalidade Libera toda memória alocada para os campos de TGenlfThenDlg.

HandleEvent

Declaração procedure Hand leEvent (var Event: TEvent); virtual;

Finalidade Trata todos os eventos gerados dentro do objeto que são estranhos ao ascendente TDialog.

Tabela 18. Eventos tratados por TGenIfThenDlg. HandleEvent

Eventos Descrição

Comandos

cmKeyListBox Comando para que a caixa de lista passe a exibir as chaves.

cmOperListBox Caixa de lista deve passar a exibir os operadores.

cmFieldListBox Caixa de lista deve passar a exibir os rótulos de campos.

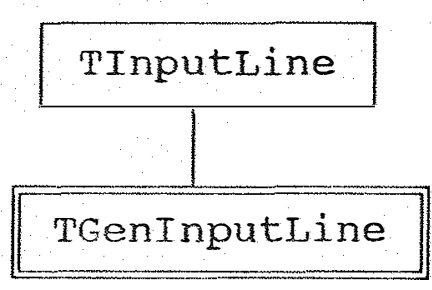

TGenInputline cria uma linha de entrada específica para digitaçāo de fórmulas para geração de variáveis. A descrição do método TFileStruct.EvaIExpr e dos procedimentos de avaliação de fórmulas presentes na unit EvalExpr fornece maiores detalhes sobre o modo de especificação dessas fórmulas.

Métodos

Error

Declaração procedure Error (Code: Integer); 
Finalidade Imprimir mensagem de erro referente devido à má especificação da fórmula . Os erros são os mesmos listados na descrição de TCondInputLine.Error.

\section{Valid}

Declaração

funct ion Valid(Command: Word): Boolean; virtual;

Finalidade Basicamente o mesmo de TCondInputLine.Valid. Os procedimentos chamados por Valid são StdForm, StdFunc e FindGenError, de modo que a expressão que retorna do objeto é aquela modificada por StdForm e StdFunc. Do mesmo modo que TCondInputLine. Valid, retorna True se NumErr de FindGenError retorna o valor 0 . CrtData

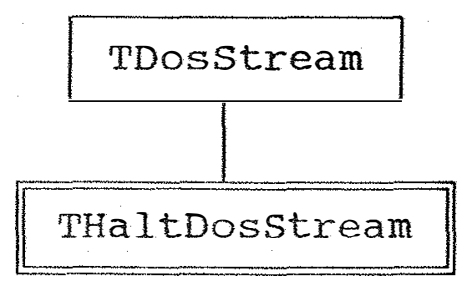

Tem a mesma finalidade de THaltStream, apenas o objeto ascendente é TDosStream ao invés de TBufStream.

Métodos

Error

Declaração

procedure Error(Code, Info: Integer); virtual;

Finalidade

Idêntica a THaltStream.Error.

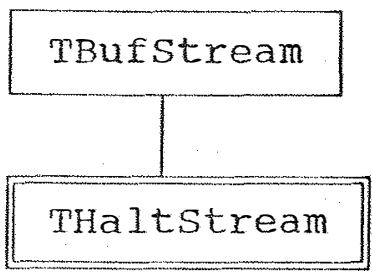


Criar um descendente que informe possíveis erros no acesso a arquivos buferizados.

Métodos

Error

Declaração

procedure Error(Code, Info: Integer); virtual;

Finalidade

Lista os erros de acesso ao arquivo. As mensagens enviadas estão na tabela 19.

Tabela 19. Códigos de erros reconhecidos por THaltStream

Code Descrição

stError $\quad$ Erro de acesso ao arquivo

stInitError Arquivo não pode ser inicializado

stReadError Leitura depois do final do arquivo

stWriteError Não pode expandir o arquivo

stGetError Tentou carregar objeto não registrado

stPutError Tentou gravar objeto não registrado

FileStru

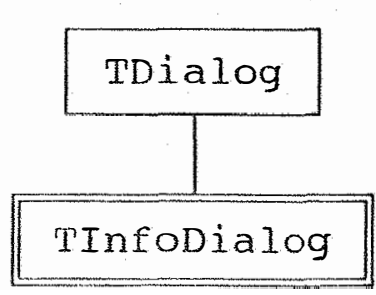

TInfoDialog tem por objetivo construir uma caixa de diálogo para informar as características gerais do arquivo de dados apontado por DataFile. 
Campos

AFile

Declaração

AFile: PDataFileObj ;

Finalidade

Ponteiro para um objeto TDataFileObj para acesso ao arquivo.

AStr

Declaração

AStr: PStrAr ray;

Finalidade

Vetor de ponteiros para cadeias cuja finalidade é possibilitar a impressão das informações sobre o arquivo.

Métodos

Init

Declaração

constructor Init (DataFvame: PathStr);

Finalidade

Constrói a caixa de diálogo e imprime as informações referentes a DataFName através de Draw.

\section{Done}

Declaração destructor Done; virtual;

Finalidade Libera a memória ocupada por TInfoDialog.

Draw

Declaração procedure Drav; virtual;

Finalidade Escreve as mensagens na caixa de diálogo.

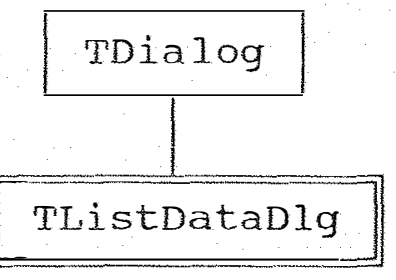


Constrói uma caixa de diálogo para coletar informações para a listagem de arquivos de dados.

\section{Campos}

AFile

Declaração

AFile: PFilestruct;

Finalidade

Ponteiro para objeto TFileStruct (correspondente a um arquivo).

ListField, ListKey, ListOper

Declaração

ListField: PCollection;

ListKey: PCollection;

ListOper: PCollection;

Finalidade Coleções que, respectivamente, armazenam rótulos referentes à campos, chaves e operadores e/ou funções.

$A B O x$

Declaração

ABox: PlistBox;

Finalidade

Ponteiro para uma caixa de lista que irá mostrar os rótulos de campos, chaves e operadores e/ou funções.

Alabel

Declaração

Alabel: PLabel;

Finalidade

Rótulo para a caixa de lista indicando o que está sendo exibido no momento.

InpField, InpCond

Declaração InpField: PView;

Finalidade Ponteiros para as linhas de entrada, respectivamente, para indicação dos campos e da condição de acesso. 
HaveKey

Declaração HaveKey: Boolean;

Finalidade Indica se existem chaves para serem utilizadas no acesso ao arquivo associado à AFile.

What List

Declaração WhatList: ListType;

Finalidade Indica qual das três coleções está sendo exibida no momento.

Métodos

Init

Declaração

const ructor Init (DatafNeme, Keyfitre: PathStr);

Finalidade

Inicializa o objeto e constrói a caixa de diálogo, informa os campos de DataFName e as chaves de KeyFName (se for o caso) disponíveis e disponibiliza duas linhas de entrada para indicação dos campos a serem tratados e a condição para a seleção de registros. Passar " para o parâmetro KeyFName indica que não estão disponíveis ou não são necessárias chaves para acesso ao arquivo.

Done

Declaração destructor Done; virtual ;

Finalidade Desaloca toda memória alocada para os campos do objeto.

\section{HandleEvent}

Declaração procedure Hand leEvent (var Event: TEvent); virtual;

Finalidade Trata todos os eventos complementares aos tratados por TDialog. HandleEvent que são, basicamente, comandos para mudanças de listas para a caixa de lista. 
Tabela 20. Eventos reconhecidos por TListDataDlg. HandleEvent
Eventos
Descrição

\section{Comandos}

cmKeyListBox Comando para que a caixa de lista passe a exibir as chaves.

cmOperListBox Caixa de lista deve passar a exibir os operadores.

cmFieldListBox Caixa de lista deve passar a exibir os rótulos de campos.

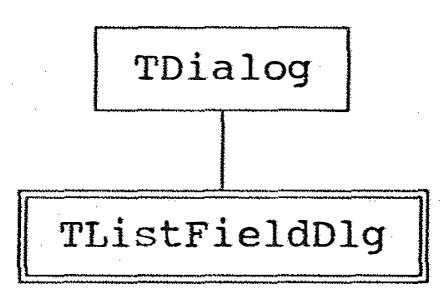

TListFieldDlg objetiva construir uma caixa de diálogo para a indicação de campos (para eliminação, seleção, etc.). Possibilita que o tipo de campo seja informado.

\section{Campos}

AFile

Declaração

Afile: PDataFileobj ;

Finalidade

Ponteiro para o objeto (correspondente a um arquivo) que irá fornecer todas as informações para a construção da caixa e outros objetos de sua propriedade.

\section{ListField}

Declaração ListField: PCollection:

Finalidade Lista de rótulos para construção de uma caixa de lista para informações acerca dos campos. 


\section{Métodos}

Init

Declaração

const ructor Init (DataPkame: PathStr; LineType: Byte; InsRad:

Boolean);

Finalidade Inicializa e constrói a caixa de diálogo e todos os demais objetos internos. DataFName fornece o nome do arquivo onde se encontram as informações sobre os campos (o arquivo será aberto e AFile será um ponteiro para ele). LineType diz respeito ao tipo de linha de entrada que será oferecida. Se for igual a 0 (zero) será colocada uma linha de entrada apropriada para a indicação de campos (objeto TFieldInputLine); se for igual a 1 (um) exibirá uma linha do tipo TInputLine. InsRad é uma variável boleana que indica a inclusão ou não de botões de rádio.

\section{Done}

Declaração

dest ructor Done; virtual;

Finalidade

Desaloca toda memória alocada para os campos do objeto.

\section{TListKeyDlg}

DataDlg

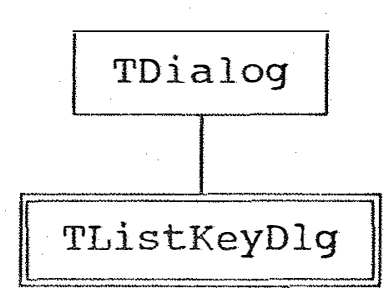

TListKeyDlg tem por finalidade construir uma caixa de diálogo para que sejam indicadas condições de acesso a arquivos e de ordenação de arquivos de chaves. Para isso implementa uma caixa de lista que informará as chaves e os operadores disponíveis no momento. 


\section{Campos}

AFile

Declaração

AFile: PFilestruct;

Finalidade

Ponteiro para um objeto TFileStruct (veja descrição do objeto TFileStruct).

ListKey, ListOper

Declaração

ListKey: PCollection;

Listoper: PCollection;

Finalidade Coleções que armazenam, respectivamente, rótulos para indicação de chaves ou de operadores e funções, respectivamente.

$A B o x$

Declaração

ABox: PListBox;

Finalidade

Ponteiro para uma caixa de lista que irá exibir os conteúdos de ListKey ou de ListOper.

ALabel

Declaração

Alabel: PLabel;

Finalidade

ALabel é um ponteiro para um rótulo que identifica a caixa de lista exibida no momento.

CondLine

Declaração

CondLine: PViev;

Finalidade

Ponteiro para uma linha de entrada que irá estabelecer a condição de acesso ou de ordenação. O tipo de linha de entrada fornecida irá depender do valor do parâmetro Cond de Init, descrito na seção de métodos.

WhatList

Declaração

WhatList: ListType;

Finalidade

Armazena o tipo de lista que está em exibição no momento. 


\section{Métodos}

Init

Declaração

const ructor Init (Keyflere: PathStr ; Cond: Byte);

Finalidade

Inicializa e constrói a caixa de diálogo abrindo o arquivo KeyFName e inicializando os campos que dele dependem. Se Cond tiver valor 0 (zero), a linha de entrada será para uma condição de acesso aos registros do arquivo FKeyName (objeto TCondInputLine); se for igual a 1 (um) a linha de entrada será para ordenação de chaves (objeto TSortInputLine); se for igual a 2 (dois) a linha de entrada será para a indicação de campos, simplesmente.

\section{Done}

Declaração

destructor Done; virtual;

Finalidade

Limpa toda a memória alocada para os campos do objeto.

HandleEvent

Declaração

,procedure HandleEvent (var Event: TEvent); virtual;

Finalidade

Trata todos os eventos gerados pela caixa de diálogo em adição aos que já são tratados por TDialog. HandleEvent.

Tabela 21. Eventos reconhecidos por TListKeyDlg.HandleEvent Eventos Descrição

\section{Comandos}

cmKeylistBox Comando para que a caixa de lista passe a exibir as chaves. cmOperListBox Caixa de lista deve passar a exibir os operadores. 


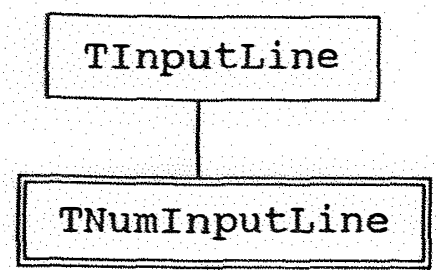

TNumInputLine tem como finalidade fornecer uma linha de entrada para digitação de valores inteiros que estejam em um determinado intervalo.

Campos

Min

Declaração Min: Longlnt ;

Finalidade Valor informado na linha deve ser maior ou igual a Min.

$\operatorname{Max}$

Declaração Max: Longlnt ;

Finalidade Valor informado na linha deve ser menor ou igual a Max

\section{Métodos}

Init

Declaração constructor Init(var Bounds: TRect; AMaxLen: Integer; AMin, AMax: Longlnt);

Finalidade Inicializa objeto TNumInputLine inicializando TInputLine com Bounds e AMaxLen. Atribui AMin e AMax, respectivamente aos campos Min e Max.

DataSize

Declaração function DataSize: Word; virtual;

Finalidade Informa que a quantidade de bytes de informação para passagem de dados é do tamanho de um inteiro longo. 
GetData

Declaração procedure GetData(var Rec); virtual;

Finalidade Devolve a informação digitada no objeto na forma de um inteiro longo.

SetData

Declaração

procedure SetData(var Rec); virtual;

Finalidade Toma um inteiro longo através de Rec, transforma-o numa cadeia e inicializa a linha de entrada com o valor.

\section{Valid}

Declaração

function Valid(Command: Wbrd): Boolean; virtual;

Finalidade

Devolve True se o número indicado na linha for um inteiro entre Min e Max.

TOnlyListBox

Fields

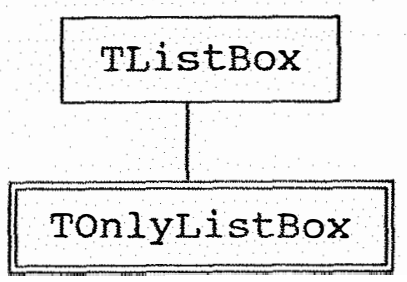

TOnlyListBox tem como finalidade construir uma caixa de lista apenas para informação, sem que haja necessidade de seleção de elementos.

Métodos

DataSize

Declaração

funct ion DataSize: Word; virtual;

Finalidade

DataSize retorna o valor zero como quantidade de bytes para informação a ser transferida, uma vez que a caixa é apenas para listagem. 
GetData -

Declaração procedure GetData(var Rec); virtual;

Finalidade Nada faz, uma vez que TOnlyListBox nada transfere.

SetData

Declaração procedure SetData(var Rec); virtual;

Finalidade Nada faz, uma vez que TOnlyListBox nada recebe para inicialização.

TSortInputLine

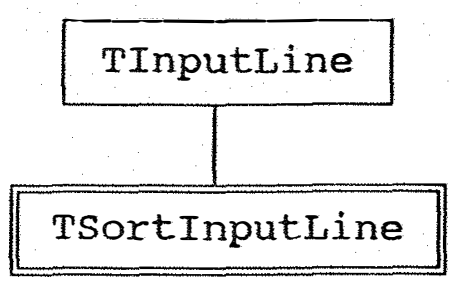

Cria uma linha de entrada destinadas às opções de ordenação das chaves de um arquivo.

\section{Métodos}

Error

Declaração

procedure Error(Code: Integer);

Finalidade

O procedimento Error lista a possível condição de erro existente na linha de entrada que estabelece as condições de ordenação. A descrição do objeto TFileStruct traz maiores detalhes acerca das possibilidades e da forma de especificação do ordenamento. Os códigos de erro são os que seguem na Tabela 22.

Valid

Declaração function Val id(Command: Word): Boolean; virtual;

Finalidade Valid faz o tratamento de erros possivelmente existentes na linha de entrada e chama Error, caso exista algum. Nesse caso, a linha de entrada não pode 
ser abandonada até que uma entrada válida seja fornecida.

Tabela 22. Códigos de erro na especificação do critério de ordenação

\section{Code Descrição}

-1 Direção ilegal de ordenamento.

-2 Posição ilegal de campo.

-3 Especificação ilegal de campo.

-4 Não existe especificação de ordenamento.

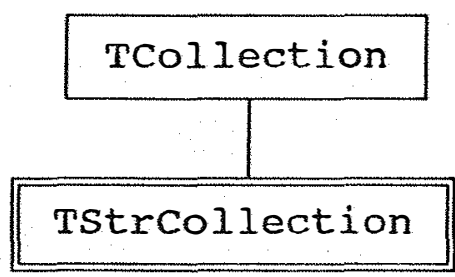

TStrCollection é um objeto descendente de TCollection, cuja finalidade é permitir a construção de coleções de PString, ou seja, de ponteiros para cadeias, uma vez que PString não é descendente de TObject.

Métodos

Freeltem

Declaração procedure Freeltem(Iten: Pointer);

Finalidade Liberar a memória ocupada por cada Item (um PString) da coleção.

\section{Gettem}

Declaração function GetItem(var S: TSt ream): Pointer;

Finalidade Através da função pré-definida TStream. ReadStr retorna um ponteiro para um ítem (PString) da coleção que está armazenada no arquivo $\mathbf{S}$. 


\section{PutItem}

Declaração

procedure Put Itom(var S: TSt rean; I tom: Pointer);

Finalidade Utiliza o procedimento pré-definido TStream. WriteStr para escrever Item no arquivo $\mathbf{S}$.

\section{TStructDialog}

CrtData

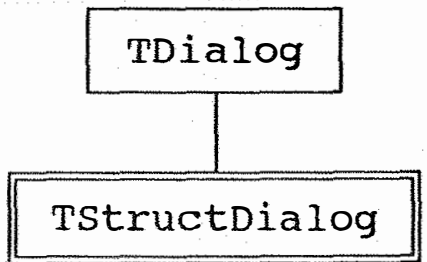

TStructDialog tem por objetivo fornecer um quadro de diálogo para a edição da estrutura dos campos.

Métodos

Init

Declaração , constructor Init(CurrStruct: Integer);

Finalidade Constrói a caixa de diálogo para edição da estrutura de número CurrStruct.

HandleEvent

Declaração procedure HandleEvent (var Event: TEvent); vi rtual;

Finalidade Trata os eventos que não são tratados por TDialog. HandleEvent. Os eventos são definidos na Tabela 23.

Tabela 23. Eventos reconhecidos por TStructDialog. HandleEvent

Eventos Descrição

Comandos

cmPrev Vai à estrutura anterior.

cmNext Vai á próxima estrutura.

cmDelete Elimina a estrutura atual. 
Tabela 23. Continuação

Eventos Descrição

Teclas

kbPgDn Pula 5 estruturas para trás.

kbDown Pula 1 estrutura para trás.

kbPgUp Pula 5 estruturas para frente.

KbUp Vai à próxima estrutura.

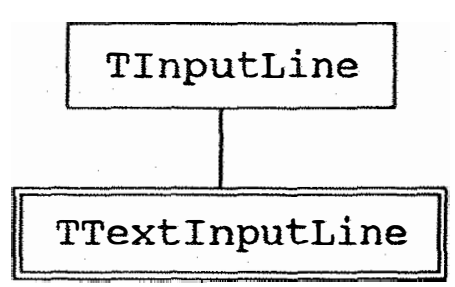

- TTextInputLine é um objeto que tem por objetivo estabelecer uma linha de entrada de texto com a condição de não aceitar linhas de entradas vazias.

Métodos

Valid

Declaração function Valid(Command: Word): Boolean; virtual;

Finalidade Valid retorna True caso a linha de entrada possua pelo menos um caractere. Desse modo a linha de entrada não pode ser abandonada enquanto não for digitado algo dentro dela. 


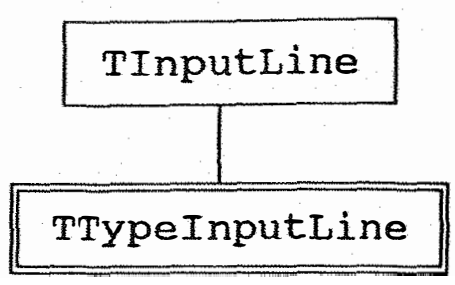

Estabelece uma linha de entrada para informação do tipo de campo (FieldType) no objeto TStruct.

\section{Métodos}

Valid

Declaração funct ion Valid(Commend: Word): Boolean; virtual;

Finalidade Retorna True se for indicado nessa linha um dos caracteres: 'N', 'C' ou 'F', válidos para essa entrada. Isso indica que essa linha somente poderá ser abandonada quando um desses caracteres for digitado.

\section{DataSize}

Declaração

funct ion DetaSize: Word; virtual;

Finalidade

Indica que a quantidade de informações a trafegar de e para o objeto TTypeInputLine é de um byte, ou seja, de um caractere.

GetData

Declaração

procedure GetData(var Rec); virtual;

Finalidade

Devolve a informação digitada na linha de entrada, do tamanho de um caractere.

SetData

Declaração

procedure SetData(var Rec); virtual;

Finalidade

Repassa o caractere ' $N$ ' para a linha de entrada. 


\section{Demais Rotinas Externas}

$\operatorname{Arc} \operatorname{Cos}$ função

GLib

Declaração

function $\operatorname{ArcCos}(x:$ Float): Float;

Finalidade

Retorna o valor do arco coseno, com $\mathbf{x}$ expresso em radianos.

ArcSin função

GLib

Declaração

function $\operatorname{ArcSin}(x:$ Float): Float ;

Finalidade

Retorna $\mathbf{o}$ valor da função arco seno para o valor $\mathbf{x}$, expresso em radianos.

BinCo função

SpecFunc

Declaração

function BinCo(n, k: Integer; var F: PFArray): Float;

Finalidade

Retorna o coeficiente binomial de $\mathbf{n}, \mathbf{k}$ a $\mathbf{k}$, como um número de ponto flutuante. $\mathbf{O}$ parâmetro $\mathbf{F}$ não é utilizado por BinCo mas é necessário para a chamada de FactLn, utilizado por BinCo. A implementação é uma adaptação da proposta por PRESS et alli (1989).

$B S t r F, I S t r F, L S t r F, W S t r F$, WStr2 função

GLib

Declaração funct ion USt rF(Nem: hord; Field: Byte): St r40;

function BStrF(Nm: Byte; Field: Byte): Str40;

function IStrF(Nm: Integer; Field: Byte): Str40;

funct ion WSt $\mathrm{r}$ (Nm: Word): Str2;

function LStrF(Nm: LongInt; Field: Byte): St r40;

Finalidade Retorna numa cadeia o número Num do tipo indicado ajustado à direita num campo de dimensão Field, com exceção de WStr2 que trabalha com cadeias de dimensão 2 somente.

CfgRec tipo

GLib

Declaração

CfgRec $=$ record

DataDi r: PathStr ;

OutDir: PathStr; 


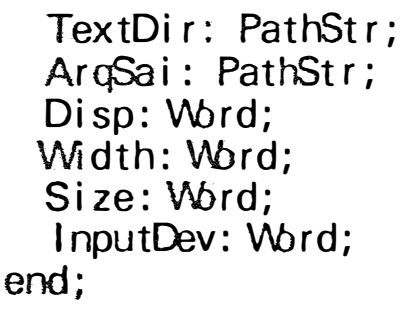

Finalidade Tipo de registro, cuja finalidade é configurar os dispositivos de entrada e saída.

Conf variável

GLib

Declaração:

$$
\begin{aligned}
& \text { Conf: CfgRec = } \\
& \text { (DataDir: ",; } \\
& \text { OutDir: ',; } \\
& \text { TextDir: ',; } \\
& \text { ArqSai : '; } \\
& \text { Disp: \$0; } \\
& \text { Width: \$0; } \\
& \text { Size: \$0; } \\
& \text { InputDev: } \$ 0) \text {; }
\end{aligned}
$$

Finalidade: Registro pré-definido, cuja finalidade é fazer a configuração de dispositivos de entrada e saída e as características com as quais será feita a saída. Os campos considerados são:

- DataDir - Diretório de onde serão lidos os dados provenientes de arquivos de tipo .TSD. Default é o diretório atual.

- OutDir - Diretório para onde serão enviadas as saídas. Default é o diretório atual.

- TextDir - Diretório onde estarão os arquivos de entrada no modo texto (tipo .TST).

- ArqSai - Nome do arquivo texto onde será colocada a saída, quando for o caso. Se ArqSai for igual a " (vazio) o nome do arquivo texto de saída em disco deve ser definido.

- Disp - Dispositivo de saída. O valor $\$ 0$ indica saída no vídeo, $\$ 1$ indica saída na impressora e $\$ 2$ indica saída num arquivo texto em disco (ocasião em que o campo ArqSai é consultado).

- Width - Indica o tamanho que terá a linha no arquivo de saída. Fazendo-se 
Width igual a $\$ 0$ a linha terá 80 colunas enquanto que Width igual a $\$ 1$ indica que a linha terá 132 colunas.

- Size - Indica o nível de compressão dos caracteres na escrita. Se Size for igual a $\$ 0$ a impressão será de 10 caracteres por polegada enquanto que se Size for igual a 1 a impressão será de 17 caracteres por polegada, ou seja, a escrita será compactada. Evidentemente, este campo somente terá sentido se a impressão for feita na impressora.

DataFileDialog procedimento

DataDlg

Declaração

procedure DataFi I eDia log (var Path: PPathStr; Cond: Boolean; st: str40);

Finalidade Este procedimento tem por finalidade obter o caminho dos arquivos de dados, cujo endereço vem através de Path. Cond estabelece se a abertura será para leitura (True) ou para escrita (False) e st fornece um título para a caixa de diálogo para a escolha do nome do arquivo. Path retorna nil se alguma condição não for satisfeita.

Date função

GLib

Declaração

function Date: Str40;

Finalidade

Retorna numa cadeia a data atual do sistema, incluindo o dia da semana.

DelBlk procedimento

GLib

Declaração procedure DelBlk(var st: string);

Finalidade Elimina de st todos os espaços em branco.

Delim variável

GLib

Declaração: Del im: set of char $=[\# 44, \# 58, \# 32, \# 13, \# 10, \# 9]$;

Finalidade: Caracteres ASCII (na ordem, vírgula, ponto e vírgula, dois pontos, espaço em branco, CR, LF, e Tab) que são considerados delimitadores para algumas rotinas que fazem o desdobramento de cadeias em partes que pos- 
suem algum significado (Tokens) ou de rotinas que fazem a leitura de algum arquivo texto.

$D S t r F D, F S t r F D$ função

GLib

Declaração function DStrFD(Nem: Double; Field, Decim: Byte): String; funct ion FStrFD(Nm: Float; Field, Decim: Byte): String;

Finalidade Retornam numa cadeia o número Num de ponto flutuante, ajustado à direita num campo de dimensão Field e com Decim casas decimais.

Eps variável

SpecFunc

Declaração

eps: Float $=10 \mathrm{e}-11$;

Finalidade

Valor que estabelece a precisão desejada na aproximação em muitos procedimentos. Essa variável é utilizada em todos os algoritmos de MARDIA \& ZEMROCH (1978), que traz uma discussão completa acerca dos seus valores mais adequados.

Factrl função SpecFunc

Declaração function Factrl/ $n$ : Integer; var F: PFAr ray; var FactNTop: Integer): Float;

Finalidade Retorna o valor de $\mathbf{n}$ !. O vetor $\mathbf{F}^{n}$ será uma tabela de valores da função fatorial já calculados e FactNTop deverá indicar quantos valores existem nessa tabela. Se numa chamada se tiver $\mathbf{n} \leq$ FactNTop então o valor será pego da tabela; caso contrário e se $\mathbf{n} \leq 32$ então a tabela será completada até $\mathbf{n}$, com o cálculo feito da forma usual, e o valor será pego da posição $\mathbf{n}+1$ da tabela; se $\mathbf{n}>32$, o valor será calculado através da função Gama. Não existe proteção contra estouro numérico no cálculo de $\mathbf{n}$ !.

Observações $\quad \mathrm{O}$ vetor $\mathbf{F}^{\wedge}$ deverá ser dimensionado para abrigar 33 valores. Na primeira chamada de Factrl, FactNTop deverá ser um valor nulo e $\mathbf{F}^{\wedge}[1]=1$. 
FileExists função

GLib

Declaração function Fil leExists (Nare: PathSt $r$ ): Boolean;

Finalidade Retorna True se $\mathrm{o}$ arquivo Name existe.

FileNameDialog função

GLib

Declaração funct ion Fi leNkmeDialog (var Filentare: PathSt $r$;

ToRead: Boolean; Mask: PathStr; Title: str40): Boolean;

Finalidade Este procedimento tem por finalidade abrir um quadro de diálogo de modo que o nome de um arquivo para abertura possa ser informado, retornando em FileName. A variável lógica ToRead indicará se o arquivo será aberto para leitura (True) ou para escrita (False). FileNameDialog retornará False se ToRead for True e o arquivo não existir no caminho indicado. Retornará False se ToRead for False e o arquivo já existe. Os parâmetros Mask e Title, respectivamente, indicam uma máscara (poderá ser, por exemplo, *.*, *.TST) para que a caixa de lista contido no quadro de diálogo liste os nomes dos arquivos que atendam à condição e fornecer um título para a caixa de diálogo.

Float tipo

GLib

Declaração: $\quad$ Float $=$ Double;

Finalidade: $\quad$ O tipo Float é o tipo utilizado para todas as variáveis de ponto flutuante dentro da biblioteca e dos demais programas. Isto indica que trocando-se o tipo para Real $($ Float $=$ Real), os números de ponto flutuante ocuparão 6 bytes cada, enquanto que para Extended (Float $=$ Extended) ocuparão 10 bytes.

FloatArrayRange tipo

GLib

Declaração: $\quad$ FloatAr rayRange $=0$. MaxFloatAr ray;

Finalidade: $\quad$ Fornece uma amplitude de variação para algumas variáveis. 
FUpCase função

GLib

Declaração function Rupcase(S: String): String;

Finalidade Retorna a cadeia $\mathbf{S}$ com todas as letras maiúsculas.

$G C F$ procedimento

SpecFunc

Declaração procedure GCF(a, x: Float; var GammCF, g|n: Float);

Finalidade Obtém o complemento da função gama incompleta de parâmetro a no ponto $\mathbf{x}$, avaliada através da sua representação como fração continuada que retorna em GammCF. O valor $\operatorname{Ln}(\Gamma(\mathbf{a}))$ retorna em gln. A implementação básica é de PRESS et al (1989).

GetIndx função

GLib

Declaração function GetIndx(n1, n2: Word): PWArray;

Finalidade Retorna um ponteiro para um vetor de inteiros positivos consecutivos, que vai de $\mathbf{n} \mathbf{1}$ a $\mathbf{n} \mathbf{2}$.

Observação , O vetor de inteiros é dimensionado e preenchido dentro deste procedimento.

GetLine função

GLib

Declaração

function GetLine(var F: Text): String;

Finalidade

GetLine retorna uma cadeia que corresponde à uma linha de um arquivo texto $\mathbf{F}$. O arquivo é lido caractere a caractere até que seja encontrado um que não pertença a Delim. A seguir, todos os caracteres diferentes dos caracteres ASCII \#13 (Carriage Return), \#10 (Line Feed) e \#26 ( ${ }^{\wedge} \mathrm{Z}$ ou final de arquivo) são incorporados a cadeia de retorno. A leitura da linha cessa assim que um desses caracteres delimitadores for encontrado.

GetTokens procedimento

GLib

Declaração

procedure GetTokens (Token: PStrCol lection; S: String) ;

Finalidade

Realiza basicamente a mesma tarefa que GetWord. A base para a retirada 
dos pedaços de texto (Tokens), entretanto, é a cadeia $\mathbf{S}$ e todos os pedaços são inseridos na coleção Token. Todos os caracteres iniciais de $\mathbf{S}$ que pertençam à Delim são descartados. Os delimitadores que identificam cada pedaço são os que compõem Delim.

Observações A coleção Token precisa ser inicializada antes que GetTokens seja chamado.

GetWord função

GLib

Declaração

funct ion Gethord(var F: Text): String;

Finalidade

Tem por finalidade retornar pedaços de texto do arquivo texto $\mathbf{F}$ que possuam algum significado (Tokens). A leitura é feita caractere a caractere até que se encontre algum diferente dos que são definidos em Delim. Os caracteres seguintes são lidos e incorporados na cadeia de retorno até que um caractere de Delim seja encontrado.

GSer procedimento

SpecFunc

Declaração, procedure GSer (a, x: Float; var Gonser, gln: Float);

Finalidade Retorna o valor da função gama incompleta com parâmetro a no ponto $\mathbf{x}$, avaliada através da sua representação por meio de uma expansão em série que retorna em GamSer. O valor $\operatorname{Ln}(\Gamma(\mathrm{a}))$ retorna através de gln.

HError variável

GLib

Declaração: $\quad$ HError : Boolean = False ;

Finalidade: Variável lógica que pode ser utilizada para a indicar se alguma condição de erro ocorre. Em geral, seu valor é alterado (torna-se True) por rotinas que imprimem mensagens de erro.

ifail variável

SpecFunc

Declaração

ifail: Integer =0;

Finalidade

ifail armazena códigos de erro gerados pelos vários procedimentos da unit 
SpecFunc. Com exceção dos valores 1 e 2, os demais são mesmos descritos por MARDIA \& ZEMROCH (1978). Os códigos constam na Tabela 24.

Tabela 24. Códigos de erros reconhecidos na obtenção dos valores das funções. ifail Descrição

\begin{tabular}{ll}
\hline 0 & Saída normal \\
1 & Parâmetro ilegal em Factrl, GSer, GammP, GammQ ou BetaI \\
2 & Numero de iterações estabelecido é muito pequeno \\
5 & A integral I foi calculada como 1 - I' onde I' foi \\
& calculada com precisão relativa eps. Assim, eps pode ser considerado agora \\
& como um parâmetro de precisão absoluta com os zeros iniciais depois do \\
& ponto decimal contando como dígitos significativos \\
& O valor da integral de probabilidade, utilizado no processo iterativo para \\
& encontrar o ponto percentual, foi obtido sob a condição 5 acima. Assim, e- \\
& xistirá uma perda de precisão neste ponto, particularmente se p é muito \\
& pequeno
\end{tabular}
$\mathrm{O}$ ponto percentual foi calculado como um múltiplo de $(1-\mathrm{x})^{ \pm 1}$ onde $\mathrm{x}$ excedeu 0.9. Portanto, existirá uma perda de dígitos significativos A integral foi calculada como 1 - 2I' onde I' é calculado para a precisão relativa eps. Deste modo, eps é agora a precisão absoluta da integral sob a condição 5 acima Foi utilizada a aproximação normal (MARDIA \& ZEMROCH, 1978) Foi utilizada uma aproximação (MARDIA \& ZEMROCH, 1978) Como na condição 14 acima, mas um valor não nulo de ifail retornou da chamada de X2Int Foi utilizada uma fórmula de interpolação harmônica de três pontos (MARDIA \& ZEMROCH, 1978) número de casos, é improvável que este resultado esteja correto. A explica- 
Tabela 24. Continuação

ifail

Descrição

ção é que o procedimento BAux muda a sua metodologia em $x=0.5,0$ que, dada a quantidade substancial de erros de arredondamento, conduz a algo que seria "numericamente descontínua em $\mathrm{x}$ " neste ponto. Isto confunde o procedimento Hyp e faz com que o valor $\mathrm{x}=0.5$ seja incorretamente retornado. Esse código indica que, provavelmente, um ponto percentual incorreto foi retornado

23

O método na condição 20 acima foi utilizado, com o problema na condição 22 ocorrendo na avaliação de 1,2 ou 3 pontos percentuais utilizados na interpolação

24

O ponto percentual de F é muito grande para ser calculado sem a ocorrência de estouro numérico. Retorna o valor -1.0

30

Erro nos graus de liberdade: $\mathrm{a} \leq 0$ ou $\mathrm{b} \leq 0$ na distribuição Beta ou $\mathrm{v} \leq$ 0 na distribuição qui-quadrado

Valor $\mathrm{p}>1$ ou $\mathrm{p}<0$ num procedimento para determinar pontos percentuais ou $\mathrm{p}$ tomando os valores 0 ou 1 , o que irá conduzir a um ponto percentual infinito

Parâmetro $\mathrm{x}<0 \mathrm{em}$ X2Int, FInt ou BInt ou $\mathrm{x}>1 \mathrm{em}$ BInt

ltMax variável

SpecFunc

Declaração

I tMax: Word = 100;

Finalidade

Estabelecer um limite para alguns processos iterativos de obtenção de valores de funções tal como propøsto por PRESS et al (1989).

KeyFileDialog procedimento DataDig

Declaração procedure KeyFileDialog(var Path: PPathStr; Cond: Boolean; st: st r 40$)$;

Finalidade Objetivo semelhante à DataFileDialog, referindo-se, entretanto, aos arquivos 
de chaves.

KeyType tipo

CrtData

Declaração

KeyType $=$ (St rKey, FloKey, FacKey)

Finalidade

Estabelecer os tipos de chave disponíveis no objeto TKey. StrKey corresponde à chaves do tipo cadeia, FloKey à chaves do tipo Float e FacKey à chaves do tipo número inteiro.

ListType tipo

DataDlg

Declaração

ListType $=($ Field, Key, Oper $)$;

Finalidade

Estabelecer tipos de listas, se de campos, de chaves ou de operadores.

LStringData, LStringDataPtr tipo

LStr

Declaração

LSt ringData $=\operatorname{ar}$ ray [1 . MaxLSt ringLength] of Char ;

LSt $r$ ingDataPt $r$ = 'LSt $r$ ingData;

Finalidade

Definem, basicamente, o vetor de caracteres que será a cadeia longa e o respectivo ponteiro.

LStringRange tipo

LStr

Declaração $\quad$ LSt r ingRange $=0$. MaxLStringLength;

Finalidade Definir a amplitude de variáveis que irão indicar o tamanho da cadeia longa.

LTrim, RTrim função

GLib

Declaração function LTrim(S: String): String;

funct ion RTrim(S: String): String;

Finalidade Retorna a cadeia $\mathbf{S}$ livre dos espaços em branco no início (LTrim) ou no final (RTrim).

MakeFieldList procedimento

DataDlg

Declaração procedure MakeFieldList (C, List: PCollection); 
Finalidade Prepara componentes para a coleção de rótulos de campos que será exibida por uma caixa de lista. MakeFieldList toma através de $\mathbf{C}$ um ponteiro para a coleção Collect de TDataFileMaker e constrói a coleção List a partir dos objetos que definem a estrutura de um arquivo (veja descrição do campo Collect do objeto TDataFileMaker) utilizando as denominações e tipos de campos.

Observações List deve ser dimensionado antes da chamada de MakeFieldList.

MakeKeyList procedimento

DataDlg

Declaração

procedure MakeKeyList (C, List: PCollection);

Finalidade Finalidade semelhante à MakeFieldList. MakeKeyList recebe através de $\mathbf{C}$ a coleção (campo KeyCol de TFileStruct) que armazena a estrutura das chaves e prepara um conjunto de rótulos para informar as chaves existentes que são incluídos em List.

Observações List deve ser dimensionado antes da chamada de MakeKeyList.

MakeNumFieldList procedimento

DataDlg

Declaração procedure MakeNumieldList (C, List: PCollection);

Finalidade MakeNumFieldList faz o mesmo que MakeFieldList mas seleciona somente os campos numéricos.

Observações List deve ser dimensionado antes da chamada de MakeNumFieldList.

MakeOperList procedimento

DataDlg

Declaração procedure MakeOperList (n1, n2: Byte; List: PCollection);

Finalidade MakẹperList seleciona os operadores e funções constantes na variável prédefinida OperArray, constrói os rótulos referentes aos ítens de $\mathbf{n} 1$ a $\mathbf{n} \mathbf{2}$ e os inclui em List.

Observações List deve ser dimensionado antes da chamada de MakeOperlist. 
Max função

GLib

Declaração funct ion $\operatorname{Max}(N 1, N R$ : Longlnt): Longlnt ;

Finalidade Retorna o maior entre dois inteiros N1 e N2.

$M a x F$ função

GLib

Declaração funct ion $\operatorname{MaxF}(a, b$ : Float ): Float ;

Finalidade Retorna o maior valor entre a e b.

MaxFloatArray constante

GLib

Declaração: $\quad$ MaxFloatAr ray $=65528$ div SizeOf (Float);

Finalidade: Dimensão máxima para matrizes de números de tipo float (real, double ou extended, dependendo da especificação inicial).

MaxLStringLength constante

LStr

Declaração

MexLSt $r$ ingLength $=65521$;

Finalidade

Estabelecer o tamanho máximo para a cadeia longa.

MaxNameSize constante

GLib

Declaração: $\quad$ MaxubreSize $=14$;

Finalidade: Estabelece o tamanho máximo para o nome associado a cada coluna no arquivo de dados.

MaxNumPointer constante

GLib

Declaração: $\quad$ MaxumPointer $=16383$;

Finalidade: Dimensão máxima para matrizes de ponteiros.

MaxNumStr constante

GLib

Declaração: $\quad$ MaxNemStr $=255$;

Finalidade: $\quad$ Número máximo de cadeias para um vetor de cadeias. 
Min função

GLib

Declaração function Min(N1, N2: Longint): Longint ;

Finalidade Retorna o menor de dois números inteiros N1 e N2.

OpenTextFile função

GLib

Declaração funct ion OpenTextFile (varhordFi le: Text ; Fi leToRead: PathSt $r$ ): Boolean;

Finalidade Retorna True se o arquivo texto de nome FileToRead for aberto com sucesso. A variável arquivo retorna em WordFile. Retorna False sempre que a variável pré-definida IOResult for diferente de zero.

OperArray variável

Declaração: OperArray: array[1..28] of string[19] =

$$
\begin{aligned}
& \text { ('UN - Log natural', } \\
& \text { 'LOG - Log decimal' } \\
& \text { 'ABS - Val. absoluto', } \\
& \text { 'INT - Val. inteiro', } \\
& \text { 'SBN - Seno', } \\
& \text { 'COS - Cosseno' } \\
& \text { 'TAN - Tangente', } \\
& \text { 'ATN - Arco tangente', } \\
& \text { 'ASN - Arco seno', } \\
& \text { 'ACS - Arco cosseno', } \\
& \text { 'EXP - Exponencial', } \\
& \text { '! - Negação', } \\
& \text { 'NEG - Negativo', } \\
& \text { 'A - Expoente', } \\
& \text { '* - Produto', } \\
& \text { '/ - Divisao', } \\
& \text { '+ - Sorro', } \\
& \text { '- - Subtração', } \\
& \text { '> - Maior que', } \\
& \text { ' }>\text { - Maior ou igual', } \\
& \text { '< - Menor que', } \\
& \text { ' }<=\text { - Menor ou igual', } \\
& '=-\mid \text { gual', } \\
& \text { ' } \infty \text { - Diferente', } \\
& \text { '\& - e (and)', } \\
& \text { 'I - ou (or)', } \\
& \text { '( - Abre parent', } \\
& \text { ') - Fecha parent'); }
\end{aligned}
$$

Finalidade: Vetor pré-definido que informa as funções matemáticas e os operadores 
disponíveis para a indicação de fórmulas matemáticas.

PadL, PadR procedimento

Declaração procedure PadR(var S: String; Field: Byte);

procedure PadL(var S: String; Field: Byte);

Finalidade Esses procedimentos têm por finalidade ajustar à direita (PadR) ou à esquerda ( $\mathrm{PadL}$ ), através do preenchimento com espaços em branco, a cadeia $\mathbf{S}$ dentro do campo Field. Caso $\mathbf{S}$ tenha comprimento superior ao campo Field, os caracteres que excedem o campo são eliminados.

PadX função

Declaração

funct ion PadX(S: string; Count, Index: Byte): string;

Finalidade

Retorna a cadeia $\mathbf{S}$ após inserir Count espaços em branco a partir da posição Index.

PByteArray tipo

GLib

Declaração:

PByteAr ray = ^TByteArray;

Finalidade: Ponteiro para vetor de bytes de uso geral. O tipo TByteArray é definido no Turbo Vision.

Power função GLib

Declaração

funct ion Pover (X, Y: Float): Float;

Finalidade

Retorna o valor $\mathbf{X}$ na potência $\mathbf{Y}$.

PPathStr tipo

GLib

Declaração:

PPathStr = 'PathStr;

Finalidade:

Ponteiro para PathStr, definido no Turbo Vision, destinado a indicação de caminhos para arquivos. 
$q m x$ variável

SpecFunc

Declaração

qnx: Float $=500$;

Finalidade

Constante, ou um seu múltiplo, utilizada nos procedimentos BInt e BPerPt. MARDIA \& ZEMROCH (1978) fazem uma discussão completa acerca da utilização dessa constante.

RegisterGLib procedimento

GLib

Declaração: procedure RegisterGib;

Finalidade: Registrar os objetos que são definidos na unit GLib.

RegisterCrtData procedimento

CrtData

Declaração

procedure RegisterCr tData;

Finalidade

Registrar os objetos da unit CrtData que poderão ser enviados para um arquivo.

RegisterDatadlg procedimento

DataDlg

Declaração

procedure RegisterDataDIg;

Finalidade

Registrar todos os objetos de DataDlg que serão enviados a arquivos.

RegisterLStr procedimento

LStr

Declaração

procedure RegisterLStr;

Finalidade

Registra todos os objetos de LStr que poderão ser enviados à arquivos.

RegisterMatrix procedimento

Matrix

Declaração

procedure RegisterMatrix;

Finalidade

Registra todos os objetos da unit Matrix.

RegisterStruct procedimento

Struct

Declaração procedure RegisterStruct ; 
Finalidade Registra o objeto TStruct para envio a arquivos.

RenameBak procedimento

GLib

Declaração procedure RenameBak (BakFi le, Fi lenbre, TempFi le: PathSt $r$ );

Finalidade $\mathrm{O}$ arquivo BakFile é apagado, FileName é renomeado para BakFile e TempFile é renomeado para FileName. Desse modo, o resultado final é que FileName passa a ser BakFile e TempFile passa a ser FileName. É um procedimento utilizado para a construção de arquivos de reserva.

Renum procedimento

CrtData

Declaração procedure Renum(C. PCollection);

Finalidade Renum recebe, através de $\mathbf{C}$, o ponteiro para a coleção de estruturas (objeto TStruct) e refaz o campo Offset. É utilizado quando essa coleção de estruturas sofre alguma modificação (eliminação de um campo, modificação nos tamanhos de campos, etc.).

$s f$ função

GLib

Declaração function sf (n: Word): Wbrd;

Finalidade A função sf retorna o número de bytes ocupados por $\mathbf{n}$ estruturas do tipo Float.

Sign função

GLib

Declaração function Sign(a, b: Float): Float;

Finalidade Retorna o valor absoluto de a com o sinal de b.

SortIndx procedimento

GLib

Declaração procedure SortIndx(var W: PFArray; var Ind: PMArray);

Finalidade Dado um vetor de números de ponto flutuante apontado por $\mathbf{W}$, este procedimento faz a ordenação dos seus componentes através do ShellSort 
e guarda a ordem respectiva no vetor Ind de inteiros positivos.

Observação $\quad \mathrm{O}$ vetor representado por Ind deverá ser dimensionado antes que SortIndx seja chamado e o seu tamanho deverá ser guardado na posição 0 (zero).

SortVecX procedimento

GLib

Declaração

procedure SortVecX(var W: PFAr ray; var Indx: PNArray);

Finalidade

Dado um ponteiro para um vetor de reais $\mathbf{W}$, os seus componentes são ordenados de acordo com a ordem estabelecida por Indx. Pode ser utilizado em conexão com SortIndx para dar a outros vetores de reais a mesma ordem relativa, ou seja, fazer uma troca correspondente, em outros vetores.

Observação

A dimensão do vetor de inteiros positivos Ind deverá ser armazenada na posição 0 (zero).

Str40, Str12, Str2 tipos

GLib

Declaração Str40 = String[40];

Str12 = String[12];

Str2 = String[2];

Finalidade Tipos de cadeias de uso geral.

StrL, StrW, StrB, Str2W, StrD função

GLib

Declaração function StrL(S: St r40): Longlnt ;

funct ion StMS: Str40): Word;

function StrB(S: Str40): Byte;

function Str2N(S: Str2): Word;

function StrD(S: String): Double;

Finalidade Retorna um número do tipo definido para a função cujo valor está armazenado em S. ValCode armazena uma possível condição de erro (ValCode $<>$ $0)$. 
StrName tipo

Declaração

St rNare = St ring MaxNbreSize ] ;

Finalidade

Estabelece o tipo para os nomes de campos.

Switch $F$ procedimento

GLib

Declaração procedure SwitchF(var $x, y$ : Float);

Finalidade Faz a troca entre os valores de $\mathbf{x}$ e de $\mathbf{y}$, ou seja, $\mathbf{x}$ retorna com o valor de $\mathbf{y}$ e $\mathbf{y}$ com o valor de $\mathbf{x}$.

TextFileDialog procedimento

DataDlg

Declaração procedure TextFileDialog(var Path: PPathStr; Cond: Boolean; st: str 40);

Finalidade Objetivo semelhante à DataFileDialog, referindo-se, entretanto, aos arquivos texto.

TFArray, PFArray tipos

GLib

Declaração: - TFAr ray $=\operatorname{ar}$ ray [1, MaxFloatAr ray] of Float ;

PFArray = ^TFAr ray;

Finalidade: $\quad$ Vetor de números do tipo Float e respectivo ponteiro para uso geral.

TIArray, PIntArray tipo

GLib

Declaração: $\quad$ TIArray = array[1..32767] of Integer ;

PIntArray = ^TIArray;

Finalidade: $\quad$ Vetor de inteiros e ponteiro para a estrutura de uso geral.

Time função

GLib

Declaração function Time: str40;

Finalidade Retorna numa cadeia a hora atual do sistema. 
TLIntArray, PLIntArray tipos

GLib

Declaração: TLIntArray = array[1. .16383] of Longlnt ;

PLIntAr ray = ^TLIntAr ray;

Finalidade: $\quad$ Mesmo que TIArray com a diferença que armazena inteiros longos (de 4 bytes).

TStrArray, PStrArray tipos

GLib

Declaração: TStrArray = array [1. MaxNerrPointer] of PString;

PSt rArray = ^TSt rAr ray;

Finalidade: $\quad$ Vetor de ponteiros para cadeias e respectivo ponteiro.

TStringArray, PStringArray tipos

GLib

Declaração: - TStringAr ray = array [1 . MaxNerrot $r$ ] of String;

PStringAr ray = ^TStringAr ray;

Finalidade: $\quad$ Vetor de cadeias para uso geral e respectivo ponteiro.

TWArray, PWArray tipos

GLib

Declaração: $\quad$ MAr ray = array[0. 32766] of hbrd;

PAr ray = ^MAr ray;

Finalidade: $\quad$ Vetor de inteiros (positivos de 0 a $64 \mathrm{~K}$ ) para uso geral e respectivo ponteiro.

Note-se que o primeiro índice do vetor é zero.

$U_{p}$ CaseStr procedimento

GLib

Declaração procedure UpCaseStr (var S: String);

Finalidade Transforma todas as letras da cadeia $\mathbf{S}$ em maiúsculas.

ValCode variável

GLib

Declaração: ValCode: Integer $=0$;

Finalidade: Armazena códigos de erro na transformação de variáveis tipo cadeia para variáveis numéricas. 


\section{Apêndice 2}

Os programas listados a seguir exemplificam a utilização de vários objetos da biblioteca.

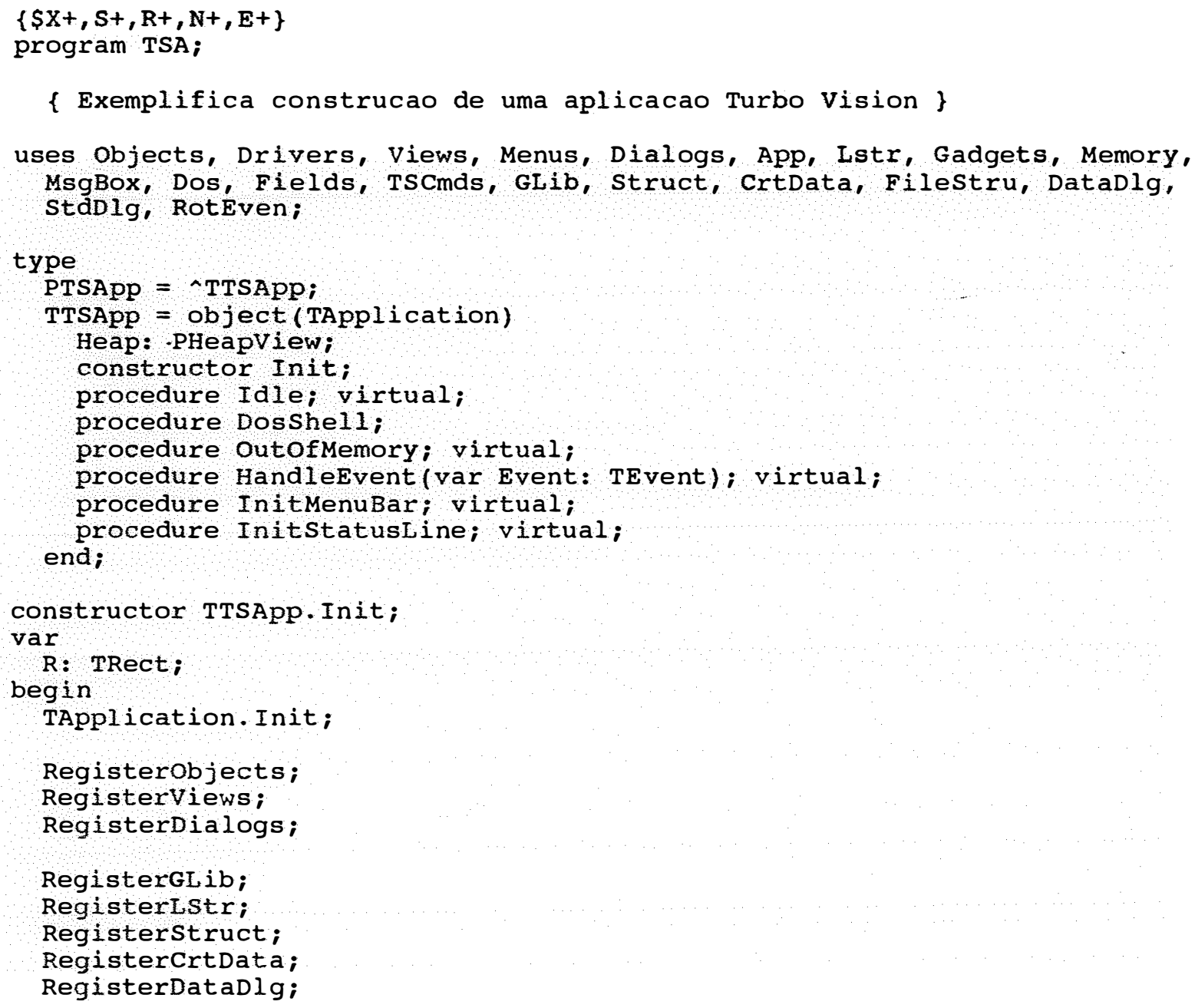




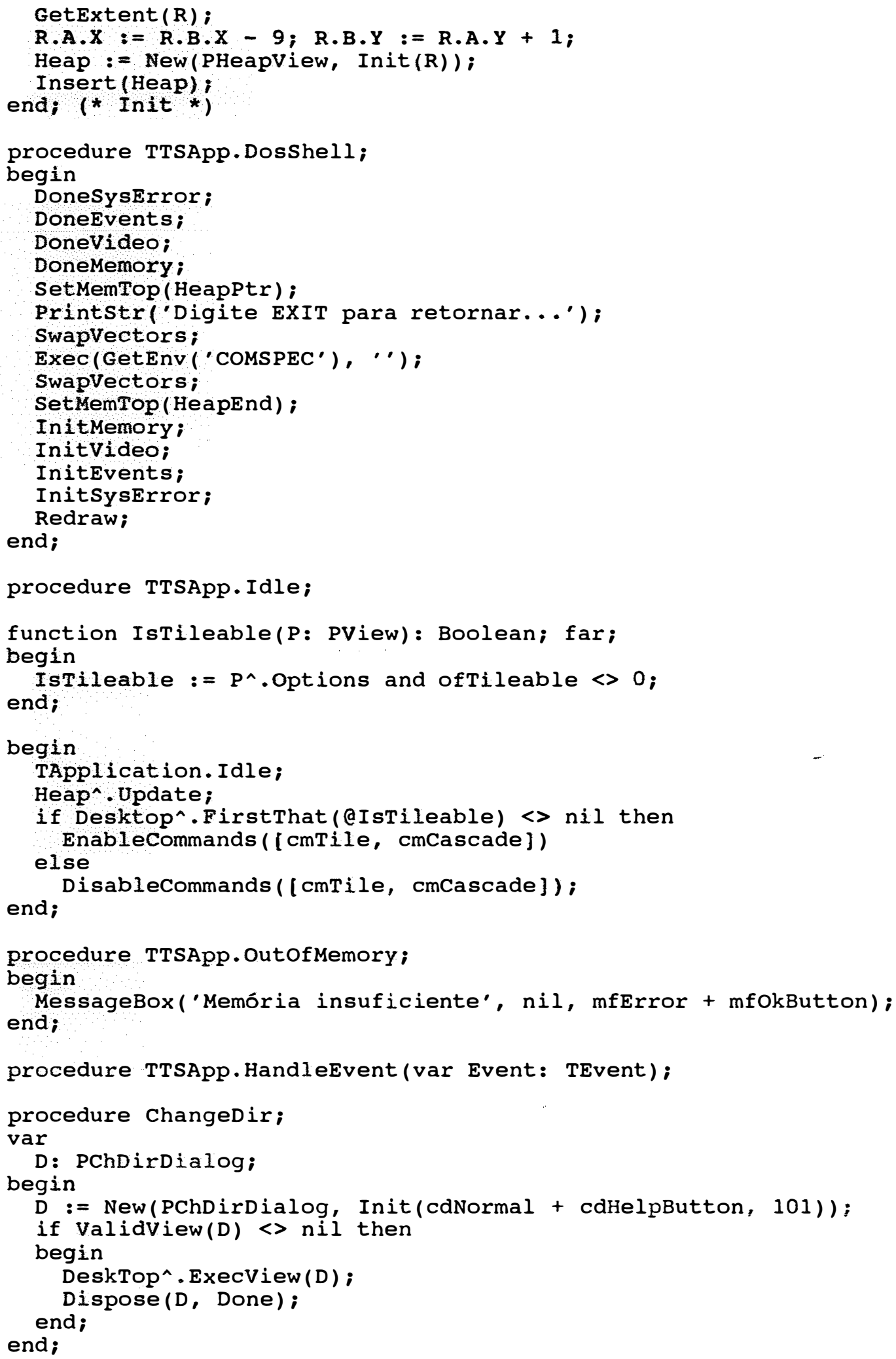




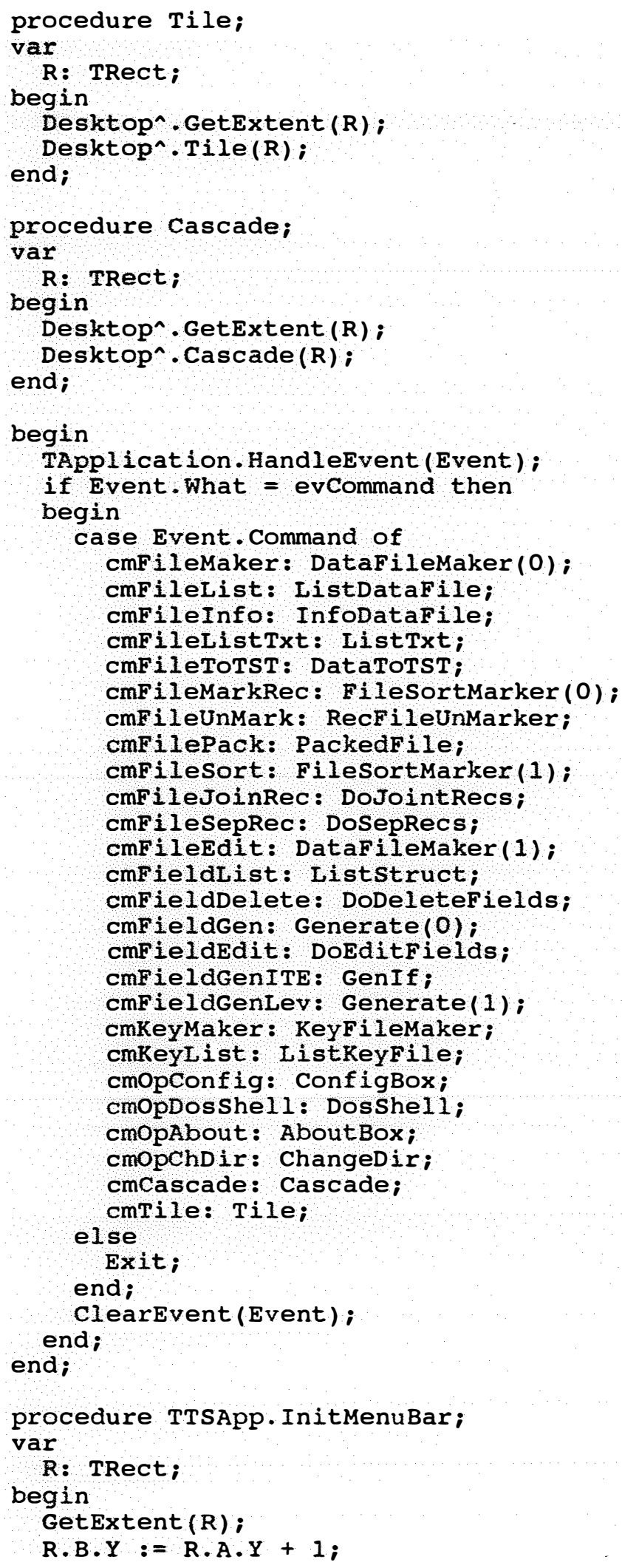


MenuBar : = Nev(PMenuBar, Init ( $R$, NewMenul

WewsubMenu('-A-rquivos', hoNoContext, NewMenul

NewItem('-C-ria/Ins...',F2', kbF2, cmFileMaker, hcNocontext,

NewItem $\left(1-\mathrm{L}-\mathrm{ista} . ., \mathrm{f}^{\prime}, \mathrm{kbF} 3, \mathrm{cmFilelist}\right.$, hcNocontext,

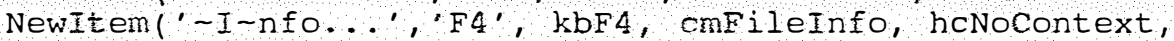

NewItem('Lista $-\mathrm{T}-\mathrm{XT}$..., F8', kbF8, cmFileListTxt, hcNocontext,

NewItem ( -G-era TST...,'Alt-F8', kbAItF8, cmFileToTST, hcNocontext,

NewItem ('Com-p-acta...', Alt-F6', kbAltF6, cmFilePack, hcNocontext,

NewItem (-O-rdena..., 'F5', kbF5, cmFilesort, holocontext,

Newlinel

Newitem(-M-arca..., , F9, kbCtrIF9, cmFileMarkRec, honocontext,

WewItem (-D-esmarca..., , kbNokey, cmFileunkark, hoNocontext,

NewItem( T-r ansfere..., F5', kbCtrlF5, cmFilejoinRec, hcNocontext,

NewItem(-S-epara..,, F4, sbCtrIF4, cmFilesepRec, hcNocontext,

NewItem ( $-J-$ unta..., , , bolokey, cmFileJoinRec, hoNoContext,

NewItem(-E-dita..., , Et-FIO, kbIILFIO, cmFileEdit, holocontext,

Newtinel

NewItem('S-a-i', Alt-X', kbAlty, cmQuit, holocontext,

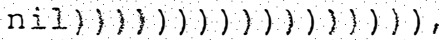

Newsublenu (-C-ampos', hcNoContext, NewMenul

NewItem( -L-ista..., 'Alt-F3', kbAItF3, cmFieldist, helocontext, NewItem (E-X-Clui..., AFG, kbCtrlF6, cmFieldDelete, hcNoContext, WewItem, -E-dita...',AIt-F9, 3bAltF9, cmFieldEdit, hcNocontext, Nexizinel

NewtEem ('-G-era...',E7', kbF7, cmFieldGen, hcNocontext,

NewItem (Gera -S-ES..., F7, kbCtrIF7, CmFieldGenITE, hcNocontext, Newilinel

NewItem ( Gera -N-iv..., ,F8, kbCtrlF8, cmFieldGenLev, hcNocontext, $\mathrm{nil}) 1111$,

NewSubkenu ( C-h-aves', hcirocontext, Newhenul

NewItem ( -C-ria..., , HL -F2, kbAltF2, cmKeywaker, hcNocontext,

NewItem ( -I-ista..., F3, kbCtrIF3, cmkeyList, hoNoContext,

$n i 1)$

NewSubMenu( -O-pçöes', hcNoContext, Newenul

NewItem ( -C-onfigura...., , F2,, kbCtrlF2, cmopConfig, holvocontext, NewItem ( DOS $-\mathrm{S}-\mathrm{heII}$, , , kbNoKey, cmopDosshell, hoNoContext, NewItem (, - D-iretorio..., , kbNokey, cmopchDir, hcNocontext, NewItem (-s-obre...,', kbNokey, cmopAbout, hcNocontext,

nil) 11$)$

NewSubMenu (Ja-n-elas', hoNoContext, NewMenul

NewItem ('-F-echa',', kbNoKey, cmClose, hcNocontext,

NewItem ( $-\mathrm{Z}-00 \mathrm{~m}^{\prime}$, , kbNokey, cmzoom, hcNocontext,

NewItem ('-M-ovimenta',', kbNokey, cmResize, hoNocontext,

NewItem ('-C-ascata',', kbNokey, cmCascade, hcNocontext,

Newttem/,-L-adrilho',', kbNoKey, cmTile, honocontext, nil) )। I)

end;

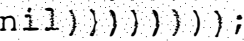

procedure TrSTpp. Initstatusine;

var

R: TRect;

begin

GetExtent (R) ;

R.A.Y $=R \cdot B \cdot Y-1$;

StatusLine $=$ New (PStatusine, Init $(R$,

NewstatusDef 10, SFFFF,

Newstatuskey ( $-F 1-A j u d a ', k b F 1$, cmHelp,

Newstatuskey ('-F2 - Cria', kbF2, CmFileMaker,

Newstatuskey (" $-F 3-$ Lista', $\mathrm{kbF} 3$, cmFileList, 
NewStatuskeyl' ^F2 - Conf', kbctrlF2, cmopconfig,

NewStatuskey(' P $_{2}$ t-F2 Cria Ch', kbAltF2, cmKeyMaker, NewStatuskey('-F7 - Gera', kbF7, cmFieldGen, Newstatuskey('-Blt-X-Sai', kbAltX, cmquit, Newstatuskey('-F10- Menu', kbF10, cmMenu, nil))) )) )), nil)) i

end;

var

TSFile: TTSApp;

begin

TSFile.Init;

TSFile.Run:

TSFile.Done;

end. \{ TSA $\}$

$\{\$ x+\}$

unit Roteven;

\{Exemplifica utilizacao de objetos p/ trat de arquivos \}

interface

procedure DataFileMaker (Mode: Byte);

procedure ListDatafile;

procecure IistTxt;

procedure DataToTST;

procedure Infodatafile;

procedure Filesortwarker (Mode: Byte);

procedure RecFileUnMarker;

procedure Packedfile;

procedure DoJointRecs;

procedure DoSepRecs;

procedure DoDeletefields;

procedure DoEditFields;

procedure GenIf;

procedure Liststruct;

procedure Generate (Mode: Byte);

procedure KeyFilemarer;

procedure ListKeyFile;

procedure ConfigBox;

procedure AboutBox;

implementation

uses Dos, Objects, Dialogs, Views, Drivers, App, MsgBox, Struct, Viewers, Memory, TSCmds, GLib, CrtData, Filestru, Datadig, Fields, StdDig;

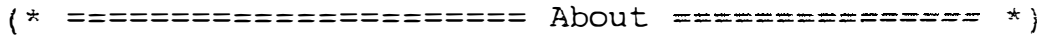

procedure AboutBox;

(* Mostra caixa de dialogo com informacoes sobre o programa *) var

D: PDialog;

Control: PView;

R: TRect; 


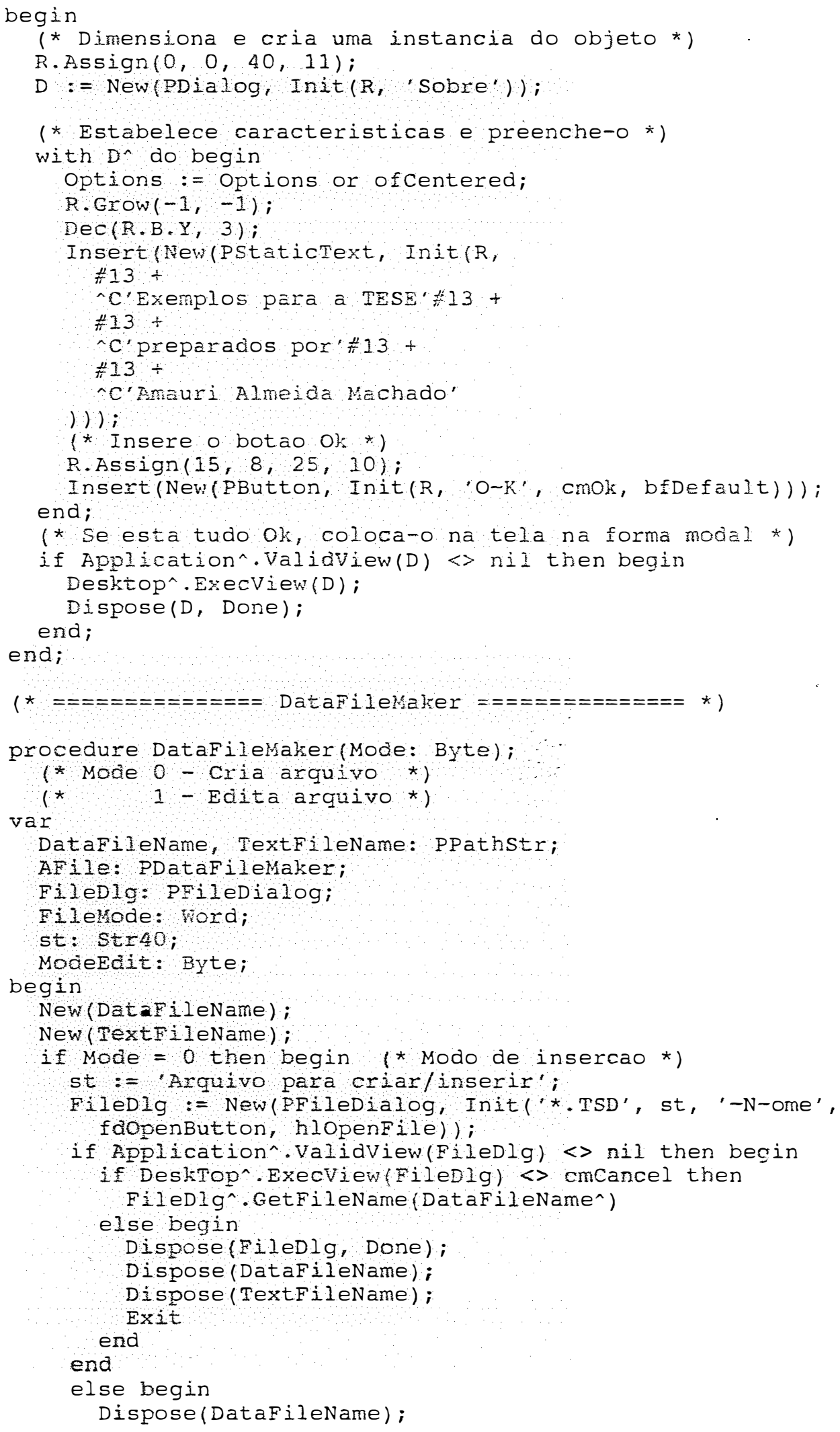




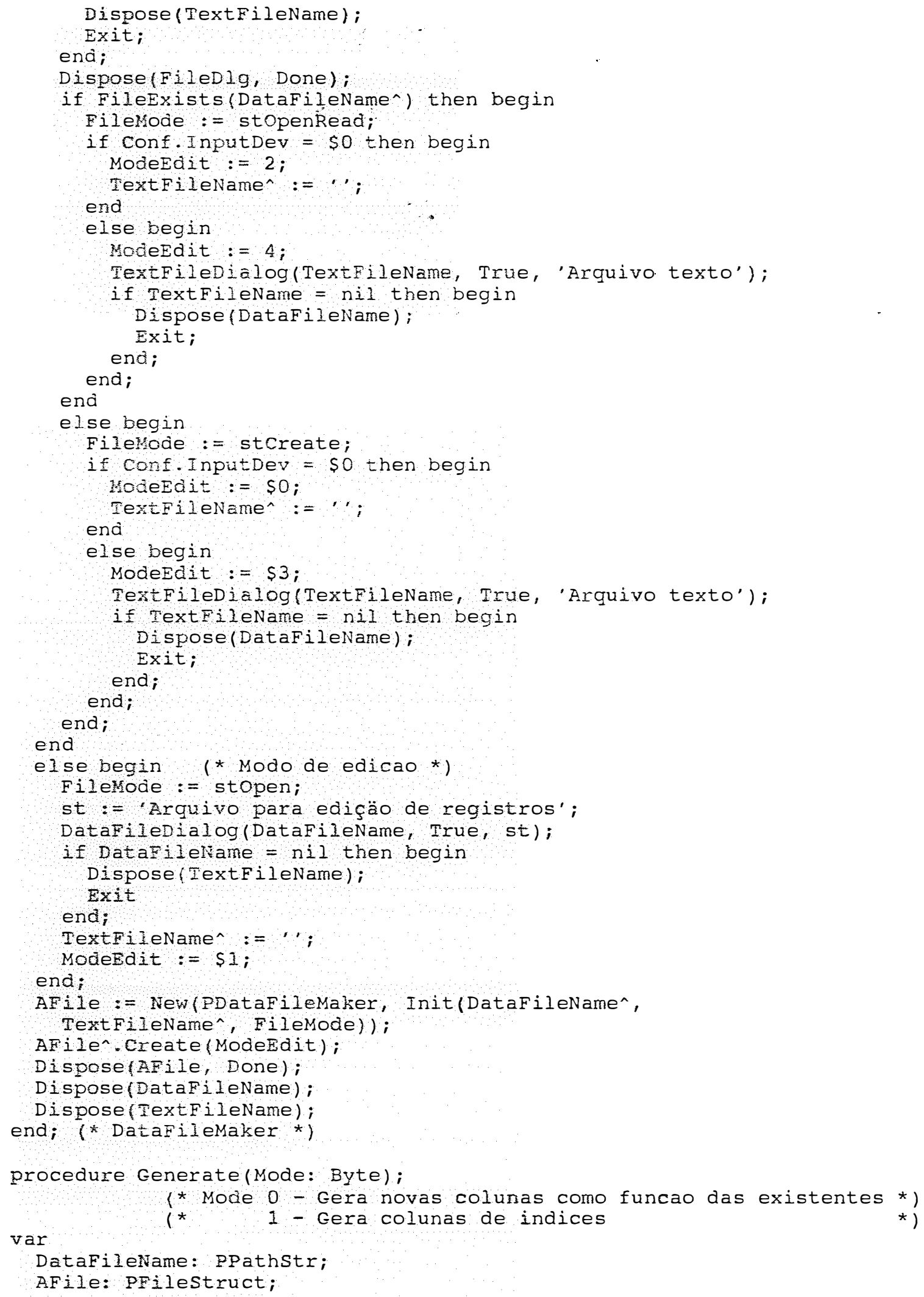




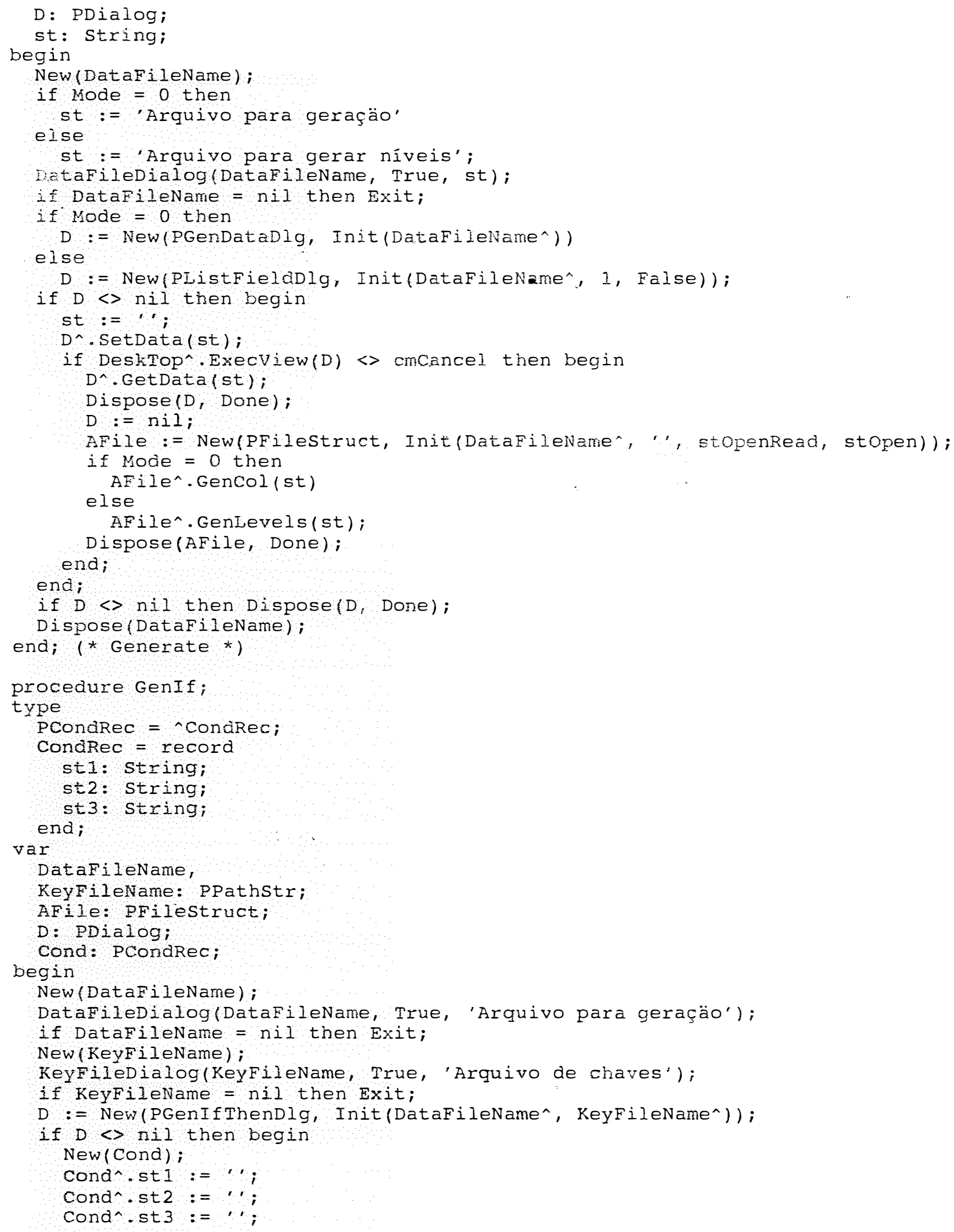




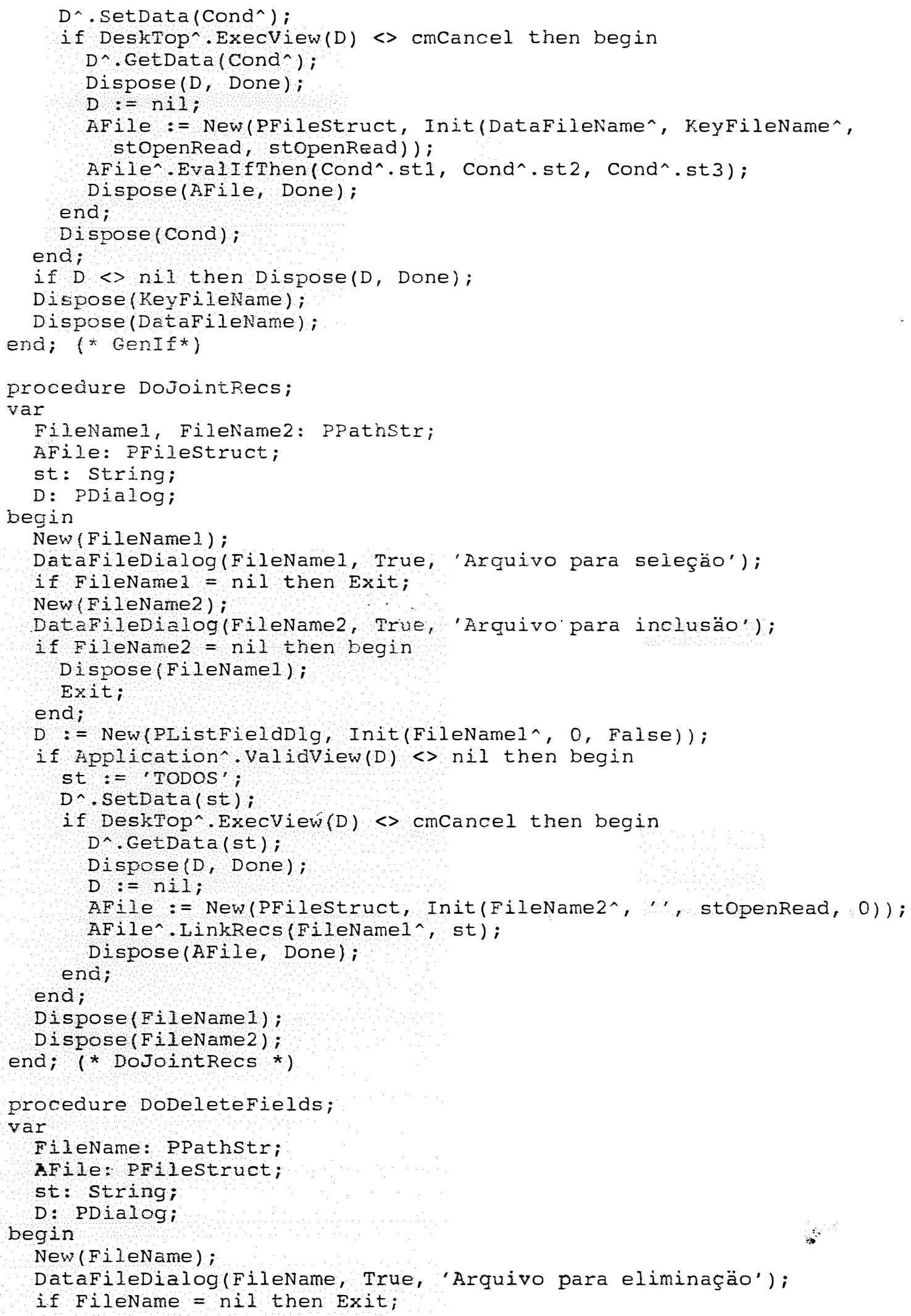




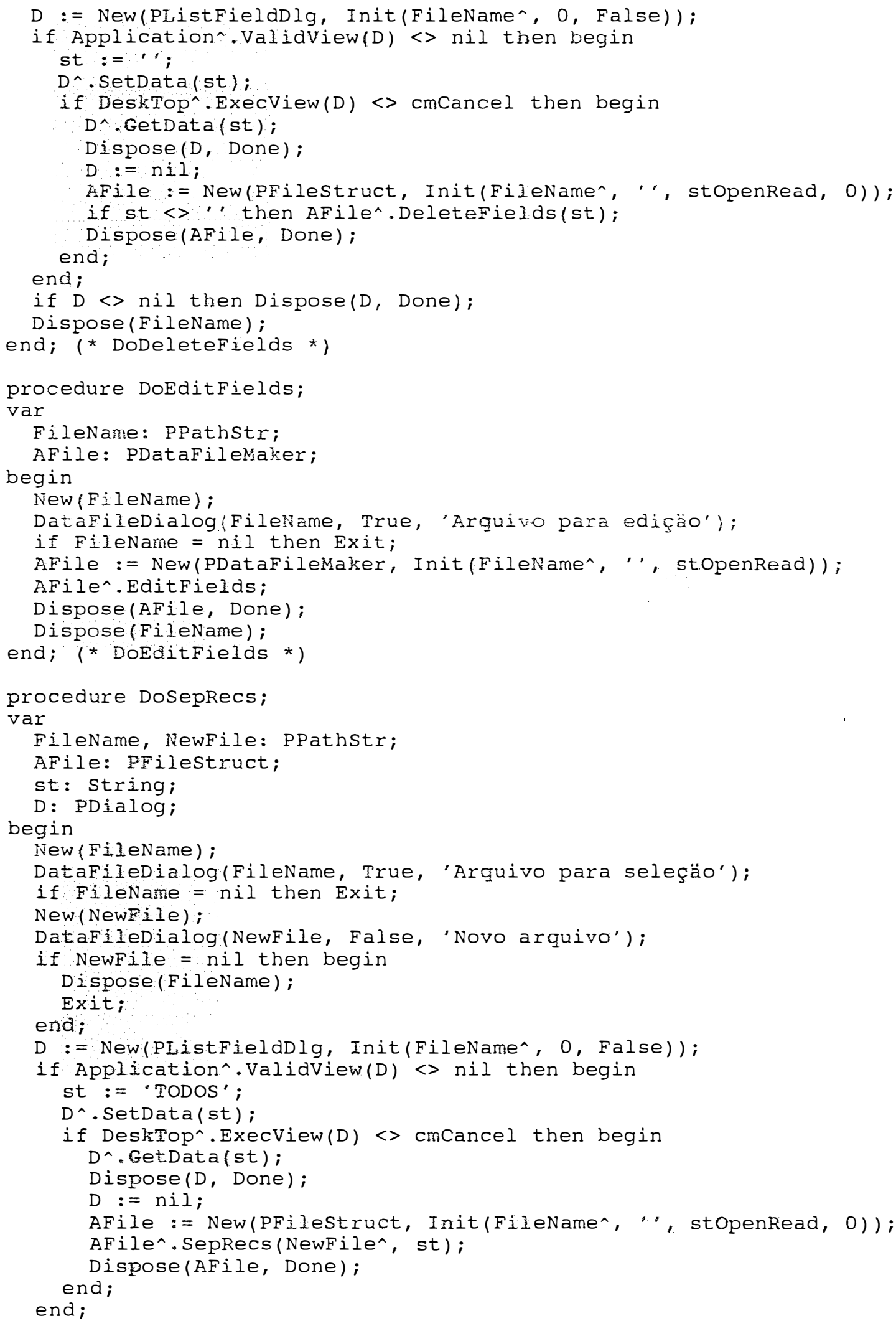



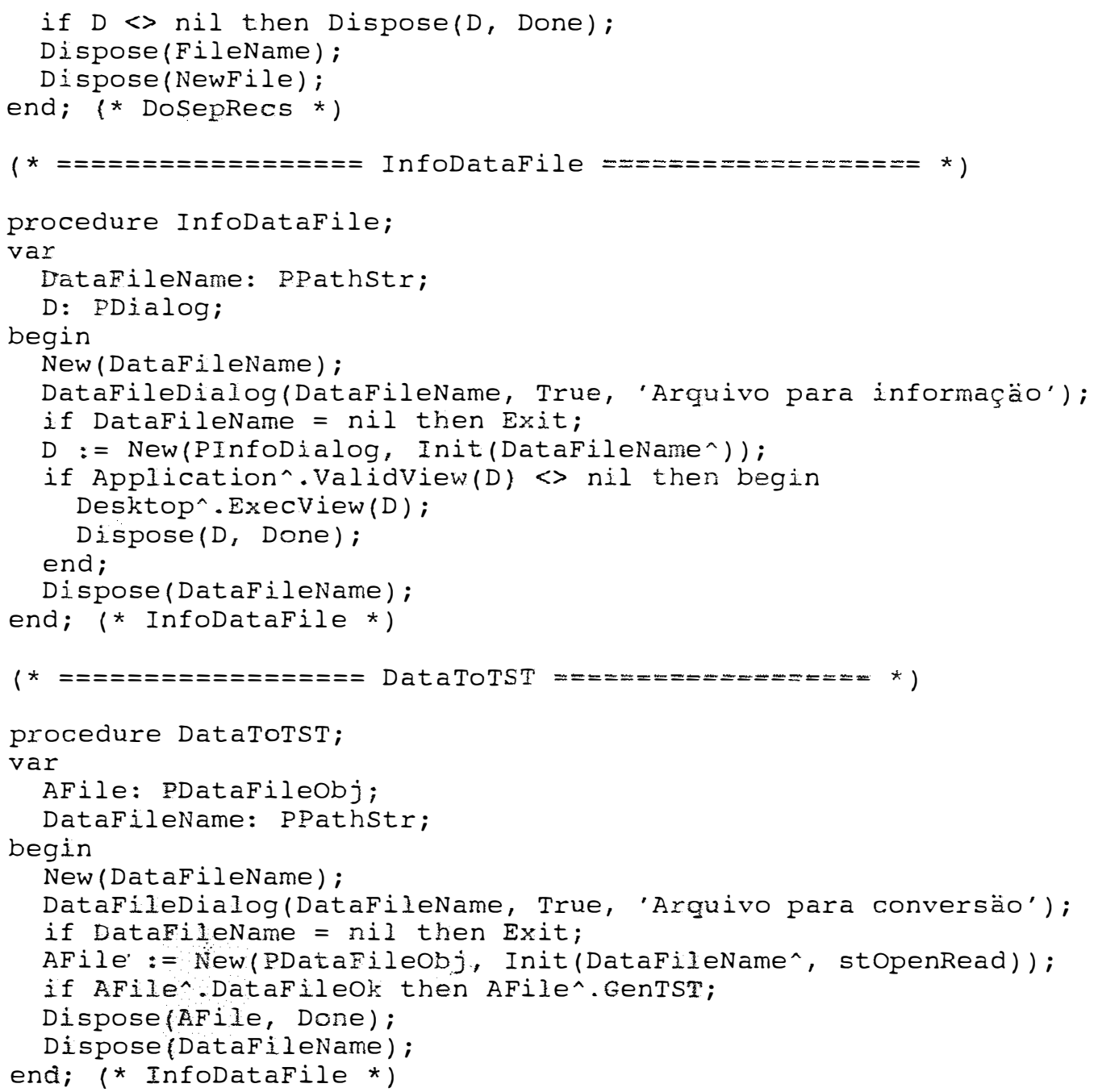

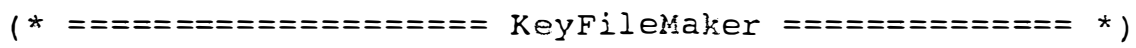

procedure KeyFilemaker;

type

KeyRec = record

st: String;

Key: Word;

end;

var

DataFileName, KeyFileName: PPathstr;

AFile: PFilestruct;

ARec: KeyRec;

D: PDialog;

begin

New(DataFileName);

Datafiledialog (Datafilename, True, 'Arquivo para retirar chaves');

if Datafilename = nil then Exit;

New (KeyFilename);

KeyFiledialog(Keyfilename, False, 'Arquivo para colocar chaves');

if KeyFilename $=$ nil then begin

Dispose(DataFileName); 


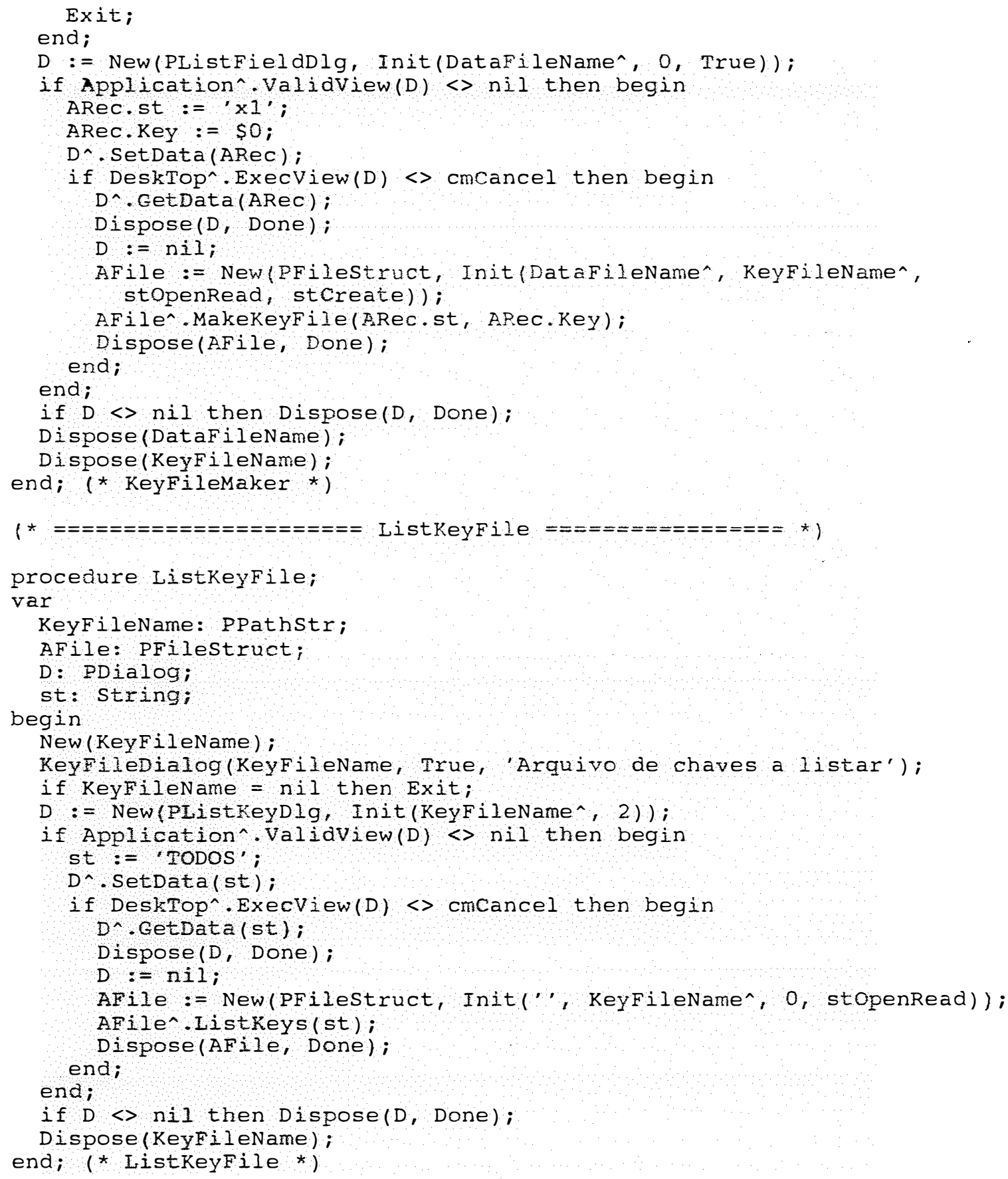




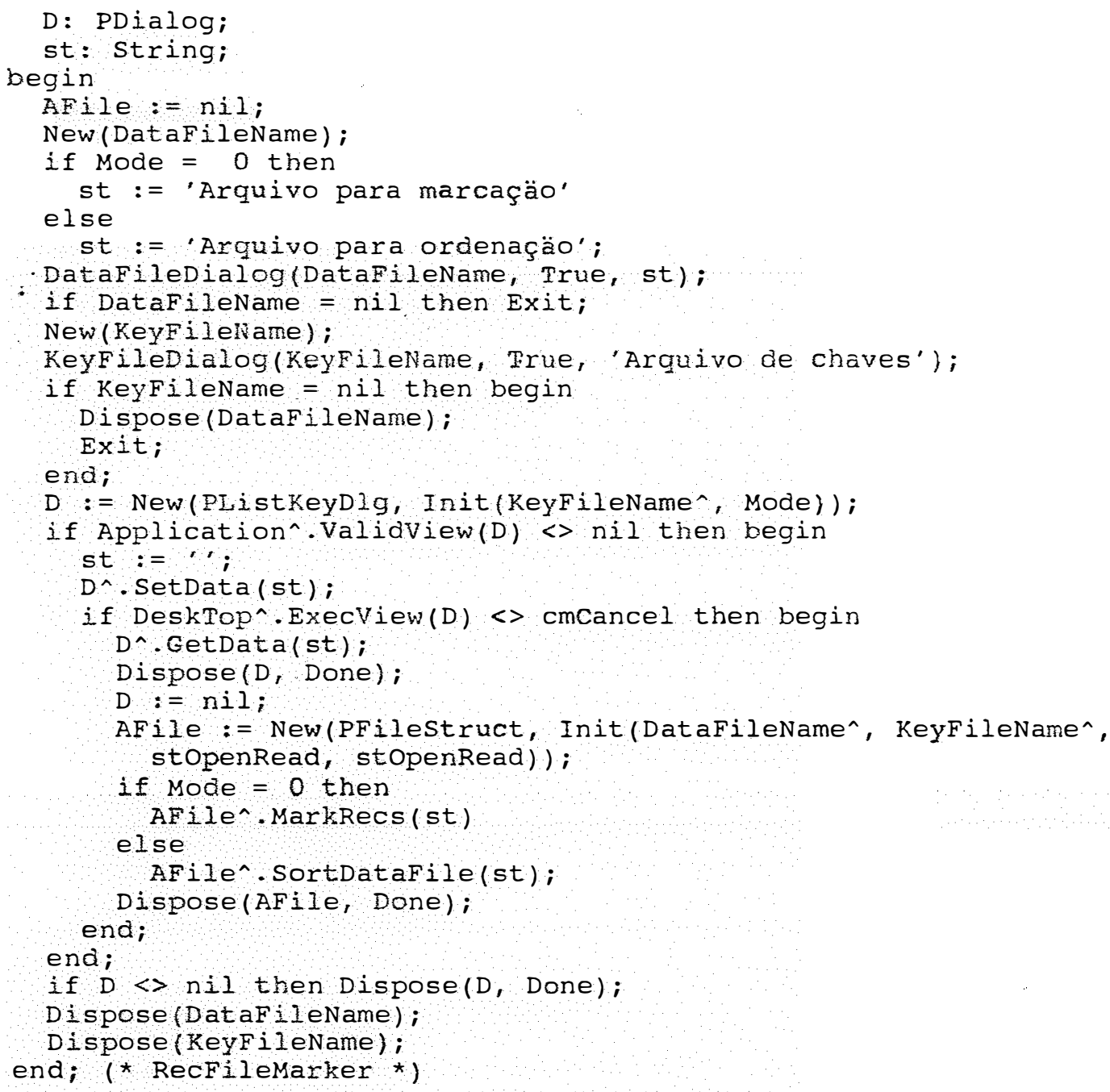


AFile: PDataFileobj;

begin

New (Datafilename);

DatafileDialog (Datafilename, True, 'Arquivo para compactar');

if DataFilename = nil then Exit;

AFile:= New(PDataFileobj, Init (DataFileName^, stopenRead));

if AFilen.Datafileok then AFile^. Deleterecs;

Dispose (AFile, Done);

Dispose (DataFilename);

end; ( * PackedFile *)

$(*=================$ ListDatafile $==========0=0$ *)

procedure ListDatafile;

type

RecData $=$ record

stField: string;

stcond: String;

end;

var

Datafilename, Keyfilename: pPathstr;

AFile: PFilestruct;

$D$ : PDialog;

ARec: RecData;

st: Pathstr;

begin

New (DataFilename);

Datafiledialog(Datafilename, True, 'Arquivo para listar');

if DataFileName = nil then Exit;

New(KeyFileName);

KeyFileDialog(KeyFilename, True, 'Arquivo de chaves');

if Keyfilename $=\mathrm{nil}$ then

st $:=\cdots$

else

st $:=$ KeyFileName^;

D := New(PListDataDlg, Init(DataFileName^, st));

with ARec do begin

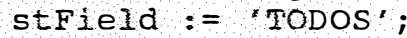

stCond : = TODOS';

end;

if Application^.ValidView(D) $<$ nil then begin

D.. SetData (ARec);

if DeskTop^. ExecView(D) $\Leftrightarrow$ cmcancel then begin

$D^{\wedge}$. GetData (AReC);

Dispose (D, Done);

$\mathrm{D}:=\mathrm{nil}$;

AFile: = New(PFilestruct, Init (DataFileName^, st, stopenRead, stopenRead)):

with ARec do AFile^. ListRecs(stField, stcond);

end;

Dispose (AFile, Done);

end;

if $D \ll$ nil then Dispose (D, Done);

Dispose (DataFilename);

if KeyFilename $<$ nil then Dispose (KeyFileName);

end, (* Listbatafile*)

procedure ListTxt;

var

Textfilename: PPathstr; 


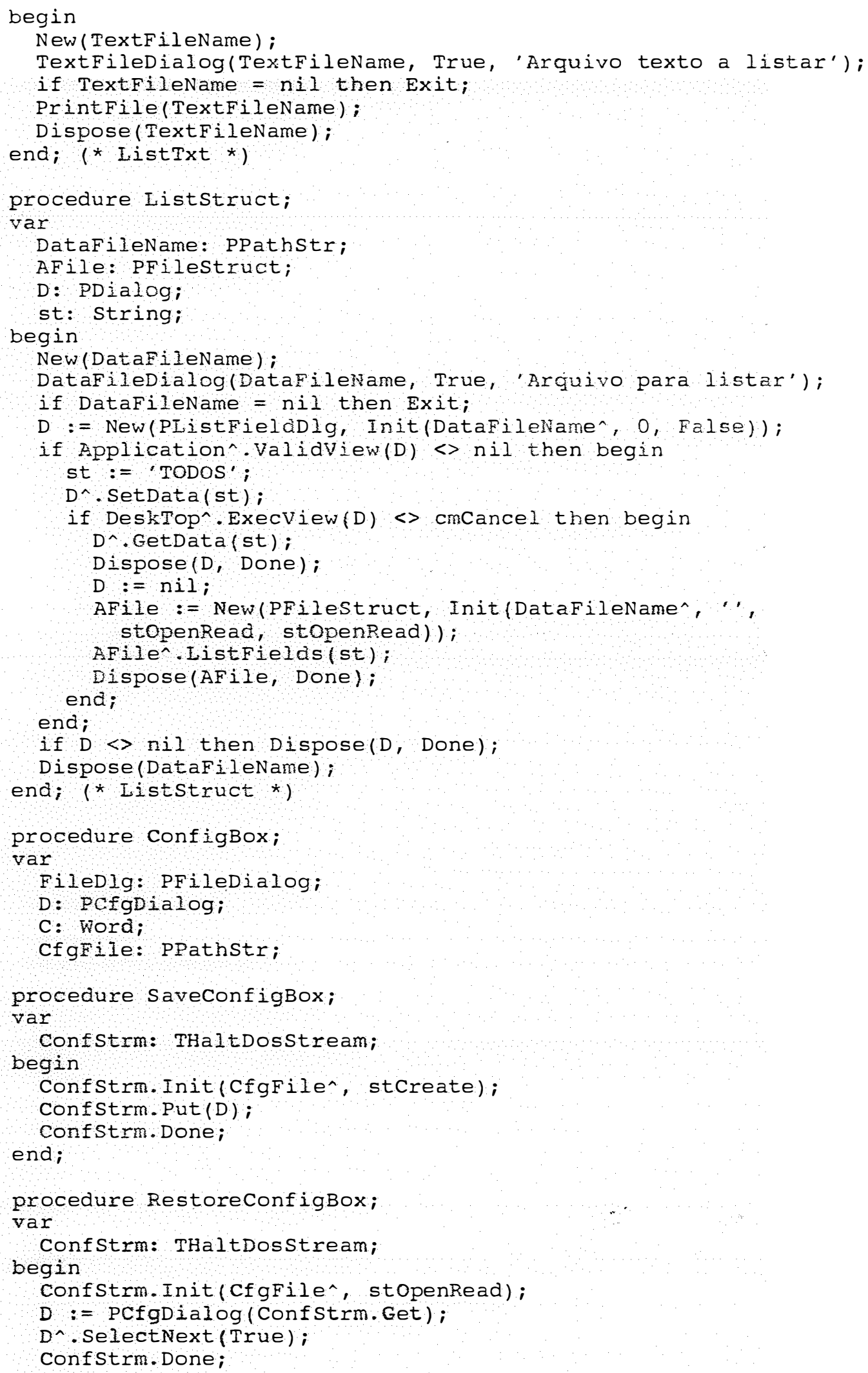


end;

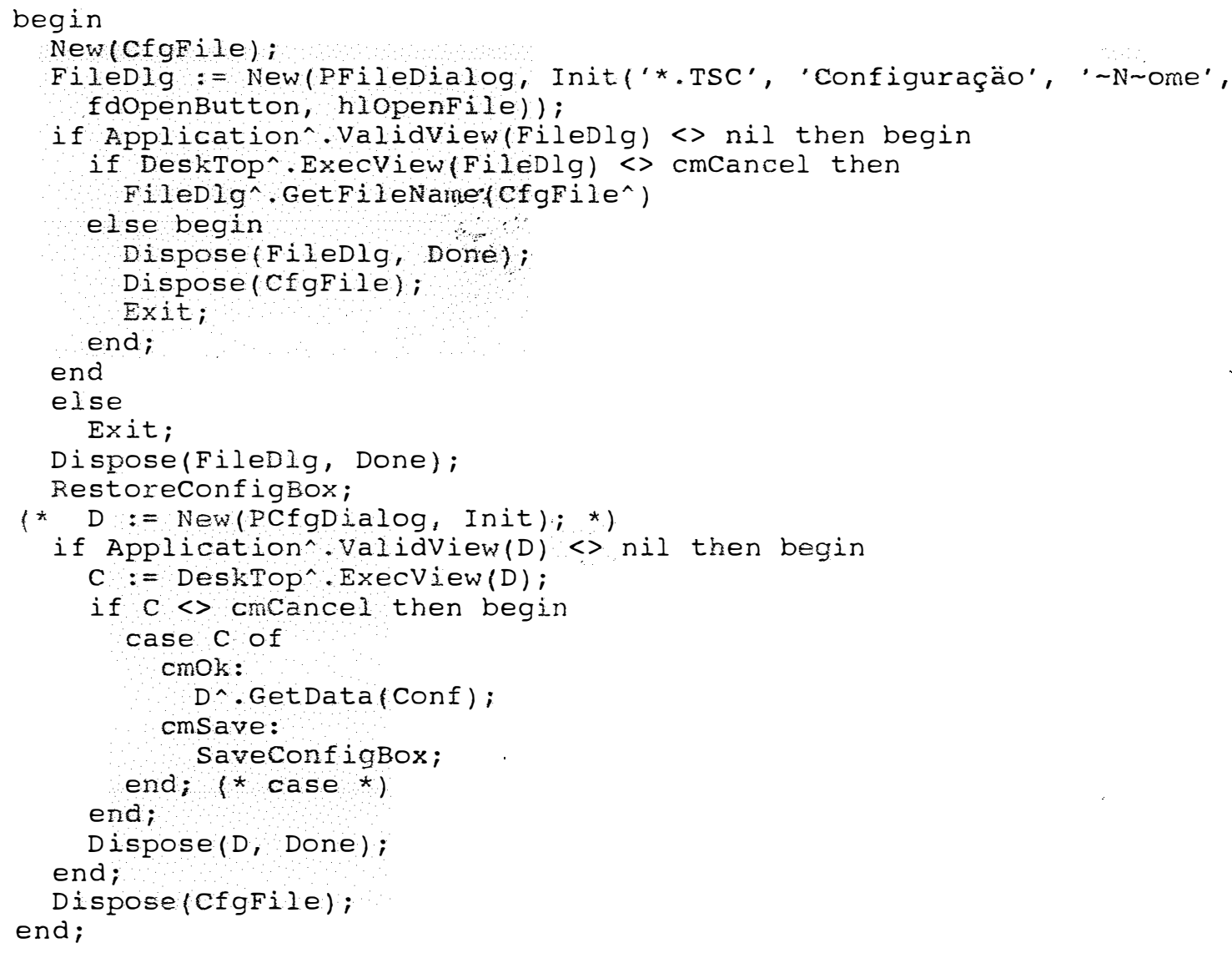

end.

$\{\$ N+, E+\}$

program TSMat;

f Exemplifica a utilizacao do objeto TMatrix e TMatcol atraves de muitos de seus metodos escolhidos por meio de um menu. Os dados necessarios sao retirados dos arquivos $\mathrm{EX}^{*}$ \}

\section{uses}

Dos, GLib, crt, objects, Matrix; const

Arq: array $[1.216]$ of Pathstr $=$

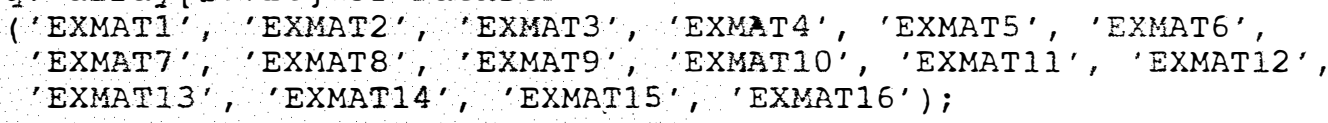


'Decomposicao por valores singulares - SVD',

'Solucao de SEL atraves do metodo de Gauss-Jordan',

'Solucao de SEL atraves da decomposicao LU',

'Solucao de SEL simetrico atraves do metodo de Cholesky',

'Solucao de SEL c/ posto completo por Householder',

'Solucao de SEL C/ posto incompleto por Householder',

'Solucao de SEL atraves da SVD',

'Solucao de SEL simetrico atraves do SWEEP',

'Inversao de matrizes atraves do metodo de Gauss-Jordan',

- Inversao de matrizes atraves da decomposicao LU',

'Inversao de matrizes atraves do SWEEP'.

'Inversa de Moore-Penrose atraves da SVD',

' Inversa G2 obtida atraves do SwEEP',

- Forma de Hermite obtida atraves do SEEP.

)$;$

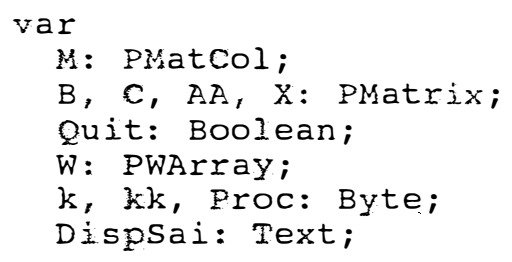




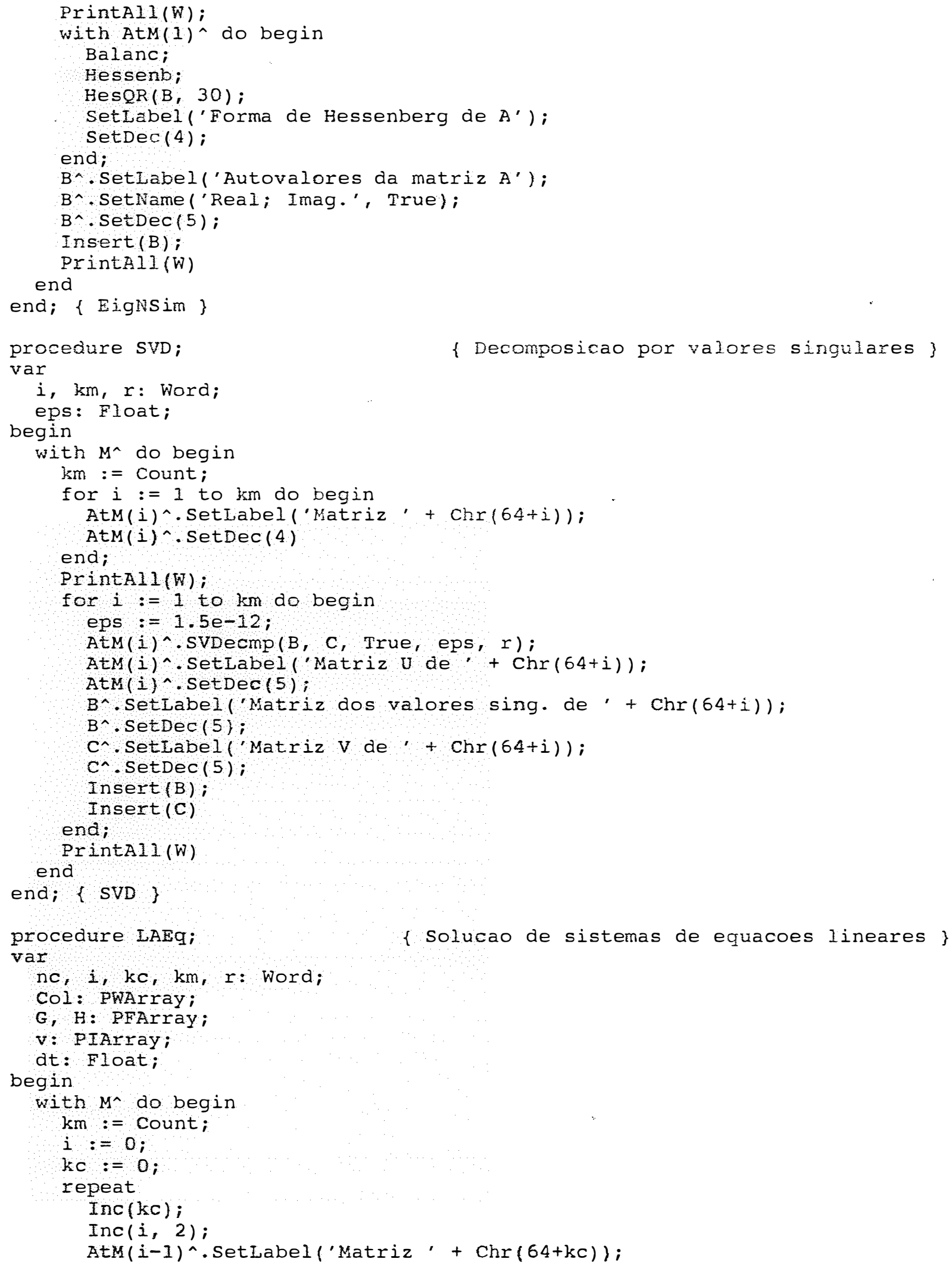




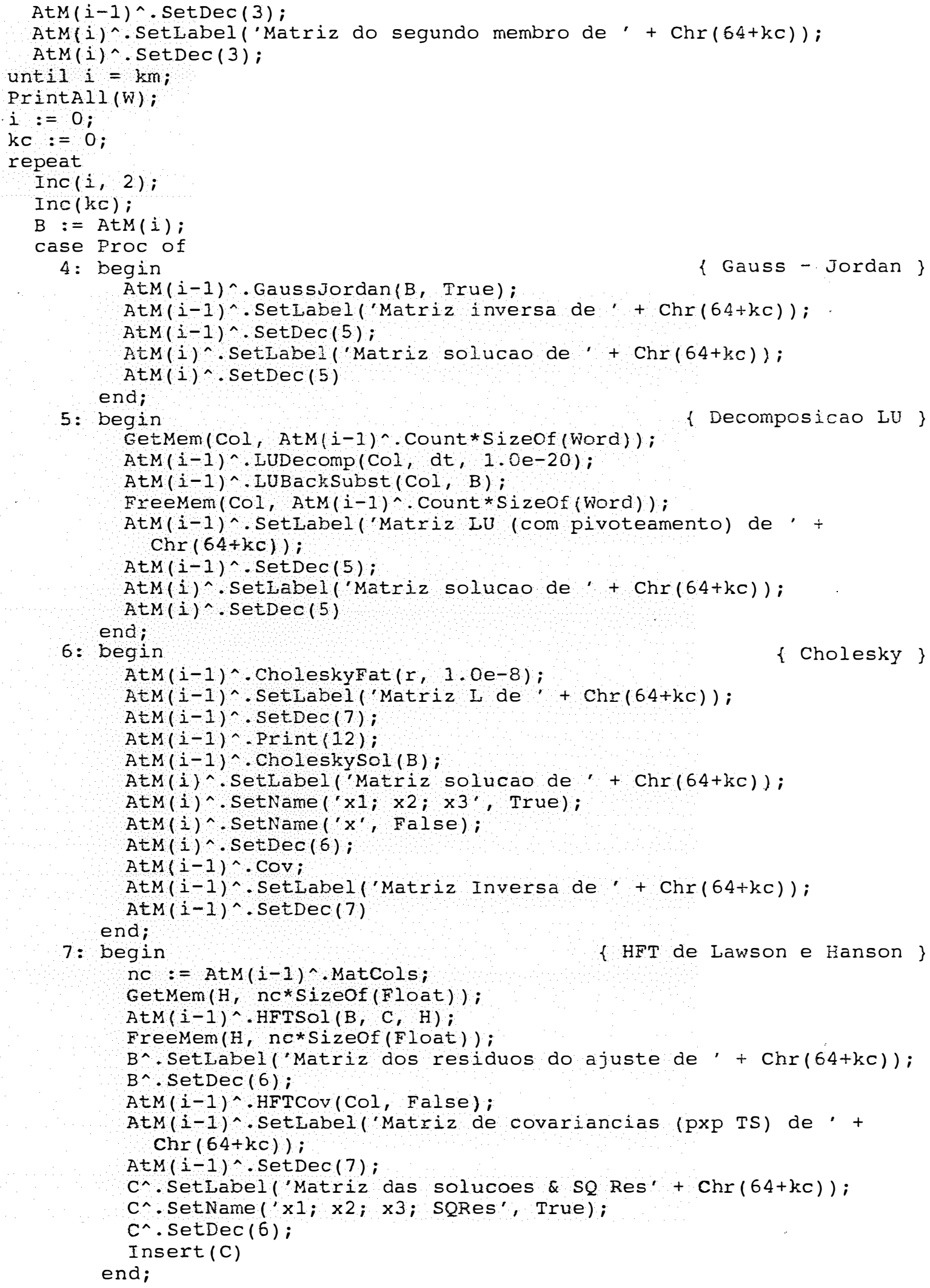




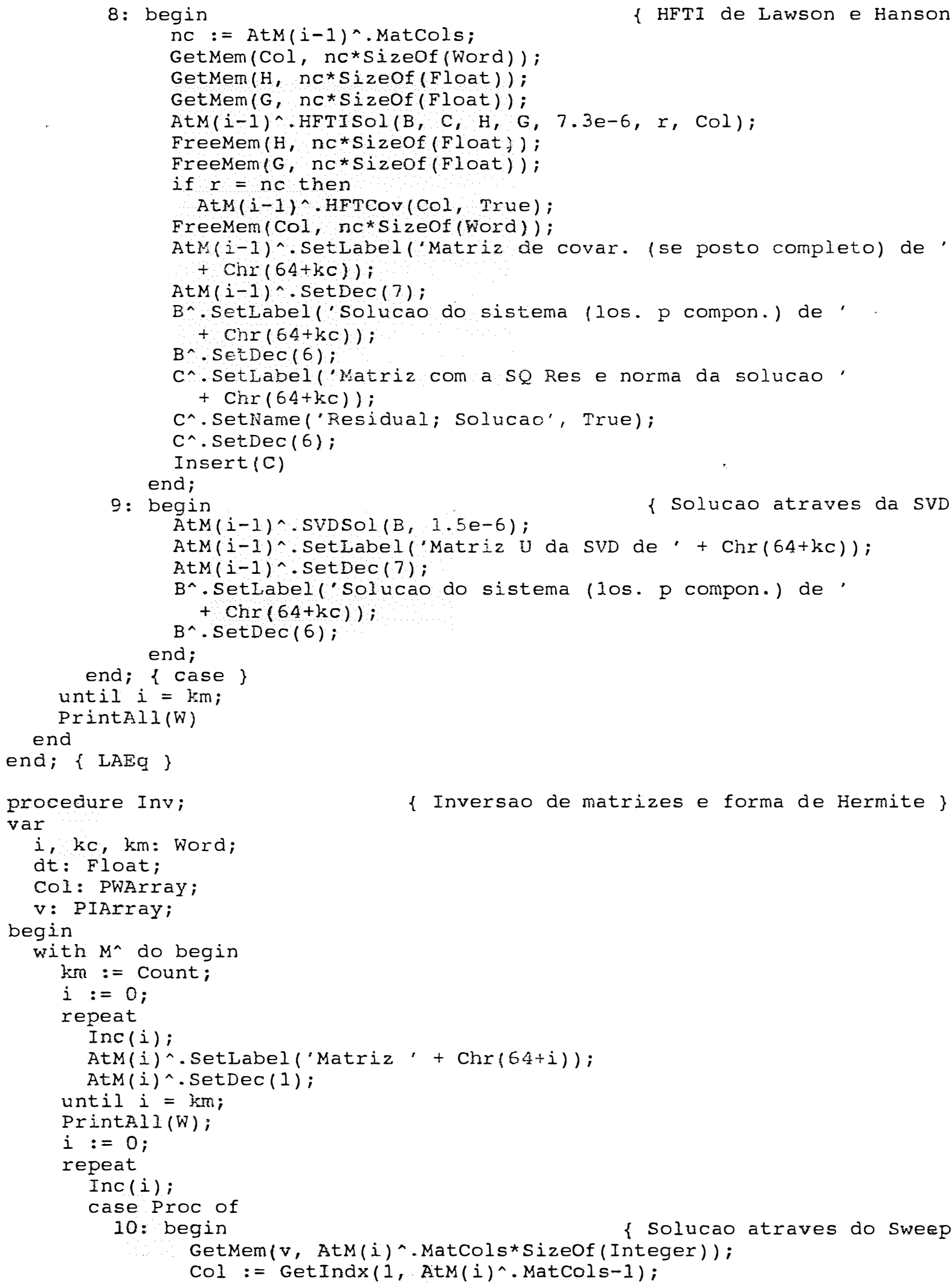




$$
\text { end; }
$$

AtM(i)^. Sweep (Col, v, 1.5e-8);

FreeMem(Col, Col^[0]*SizeOf(Word));

FreeMem(v, AtM(i)^.MatCols*Sizeof (Integer));

$\operatorname{AtM}(i) \wedge$. SetDec (4);

AtM(i)^. SetLabel('Matriz com a solucao de' +

$\operatorname{Chr}(64+i))$

11: begin

AtM(i)^.GaussJordan(B, Faḷse);

$\{$ Inversao por Gauss Jordan \}

AtM(i)^.SetLabel ('Matriz Inversa de' $+\operatorname{Chr}(64+i))$;

$\operatorname{AtM}(i)^{\wedge}$. SetDec(5)

end;

12: begin

$\{$ Inversao pela decomposicao LU \}

GetMem(Col, AtM(i)^.Count*Sizeof(Word));

$\operatorname{AtM}(i)^{\wedge}$. LUDecomp (Col, dt, 1.5e-10);

$\operatorname{AtM}(i)^{\wedge}$. LuInverse $(B, C o l)$;

AtM(i)^.SetLabel ('Matriz LU (com pivoteamento) de' $+\operatorname{Chr}(64+i))$;

$\operatorname{AtM}(1) \wedge$.SetDec (4);

FreeMem(Col, AtM(i)^. Count*Sizeof (word));

$\mathrm{B}^{\wedge}$. SetLabel ('Matriz inversa de ' $\left.+\operatorname{Chr}(64+i)\right)$;

$\mathrm{B}^{\wedge}$. SetDec (5);

Insert ( $B$ )

end;

13: begin $\{$ Inverte pelo sweep \}

$\operatorname{GetMem}\left(v, \operatorname{AtM}(i)^{\wedge} \cdot \operatorname{MatCol} s^{\star} \operatorname{SizeOf}(\right.$ Integer));

$\operatorname{Col}:=\operatorname{Get} \operatorname{Indx}(1, \operatorname{AtM}(1) \wedge$.MatCols $)$;

Atm (i)^. Sweep (Col, v, $1.5 e-8)$;

FreeMem (Col, Col^ $[0\} *$ SizeOf (Word));

FreeMem(v, AtM(i)^.MatCols`Sizeof(Integer));

$\operatorname{AtM}(1) \wedge$. SetDec (7);

$\operatorname{AtM}(i) \wedge$. SetLabel ('Matriz inversa de $+\operatorname{Chr}(64+i)$ ) end;

14: begin \{ Inversa de Moore Penrose atraves da SVD \} AtM(i)^.MorePenrose (B, 1.5e-8);

AtM(i)^. SetLabel ('Matriz U de'+Chr(64+i));

$\operatorname{AtM}(i) \wedge$. SetDec (4);

$\mathrm{B}^{\wedge}$. SetDec (5);

$\mathrm{B}^{\wedge}$. SetLabel('Matriz inversa de Moore Penrose de '+ $\operatorname{Chr}(64+i)$ ); end;

Insert (B)

15: begin

\{ Inversa G2 atraves do Sweep \}

$\operatorname{GetMem}\left(v, \operatorname{AtM}(i)^{\wedge}\right.$.MatCols*SizeOf(Integer));

Col: $=\operatorname{GetIndx}(1, \operatorname{AtM}(i) \wedge$ MatCols $)$;

$\operatorname{AtM}(i) \wedge$.MatCopy $(X)$;

$\operatorname{AtM}(i) \wedge$. Sweep (Col, v, 1.5e-8);

$\operatorname{AtM}(i) \wedge . G 2 \operatorname{Inv}(B, v)$;

$B^{\wedge}$.Mult ( $X, C$, True);

Dispose ( $X$, Done);

FreeMem (Col, Col^[0]*SizeOf (Word));

FreeMem(v, AtM (i)^.MatCols*Sizeof (Integer));

$\operatorname{AtM}(i) \wedge$. SetLabel (Matriz + $\operatorname{Chr}(64+i)+$

operada pelo SWEEP');

$\mathrm{B}^{\wedge}$. SetDec (2);

B^. SetLabel ('Matriz inversa G2 de' + $\operatorname{Chr}(64+i)$ );

C.. SetDec(1);

C^.SetLabel ('Forma de Hermite de ' + $\operatorname{Chr}(64+i)+$ - pelo produto');

Insert (C);

Insert (B); 
end;

16: begin \{Forma de Hermite atraves do Sweep \}

GetMem(v, AtM(i)^.MatCols*Sizeof (Integer));

Col:= GetIndx (1, AtM(i)^.MatCols)

AtM(i)^. Sweep (Col, v, $1.5 e-8)$;

$\operatorname{AtM}(i) \wedge$ Hermite(C, v);

FreeMem (Col, Col^\{0\}*SizeOf (Word));

FreeMem(V, AtM(i)^.MatCols*Sizeof (Integer));

AtM(i)^.SetLabel('Matriz! + Chr $(64+i)+$

- operada pelo SWEEP');

C. SetDec (1);

C^. SetLabel('Matriz de Hermite de ' $+\operatorname{Chr}(64+i)$ ); Insert (C);

end;

end; $\{$ case $\}$

until $i=\mathrm{km}$;

PrintAll (W)

end

end; $\{$ Inv $\}$

begin

if Paramcount $=0$ then begin

Writeln('Este programa necessita de um arquivo para impressao'); Exit

end

else

Conf. Arqsai : = Paramstr (1);

Quit : = False;

repeat

kk : = 10;

GetMem ( $w, k k * S i z e O f(W o r d))$;

\{ Dimensao de cada coluna para listagem \}

for $k:=0$ to $k k-1$ do $W^{\wedge}[k]:=12$;

\{ Instancia do objeto para a colecao de matrizes \}

M:= New(PMatCol, $\operatorname{Init}(6,2))$;

Clrscr;

writeln;

writeln;

writeln ('

writeln('

writeln('

writeln("

writeln!

writeln('

writeln('

writeln ('

writeln('

writeln('

writeln! '

writeln('

writeln ('

writeln('

writeln('

writeln('

writeln.'

0: Encerra ');

1: Autovalores de uma matriz simetrica');

2: Autovalores de uma matriz nao simetrica');

3: Decomposicao por valores singulares');

4: Solucao por Gauss-Jordan') ;

5: Decomposicao LU') ;

6: Solucao por Cholesky');

7: Solucao de $M Q$ de posto completo por Householder');

8: Solucao de $M Q$ de posto incompleto por Householder');

9: Solucao de MQ pela SVD');

10: Solucao atraves do Sweep') ;

11: Inversao por Gauss-Jordan') ;

12: Inversao pela decomposicao LU');

13: Inversao pelo Sweep');

14: Inversa de Moore Penrose atraves da SVD');

15: Inversa G2 atraves do Sweep');

16: Forma de Hermite atraves do Sweep');

writeln;

repeat 


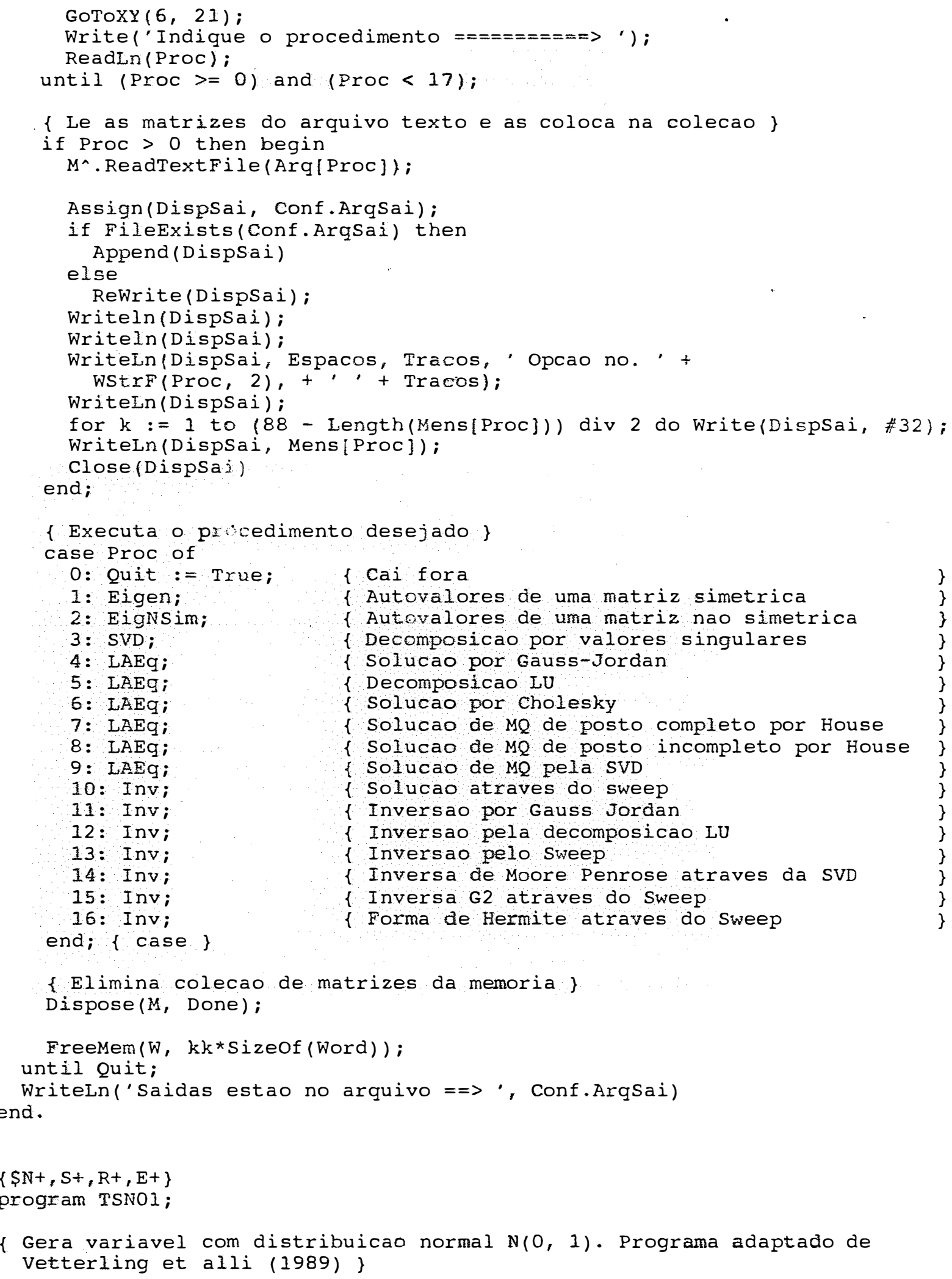




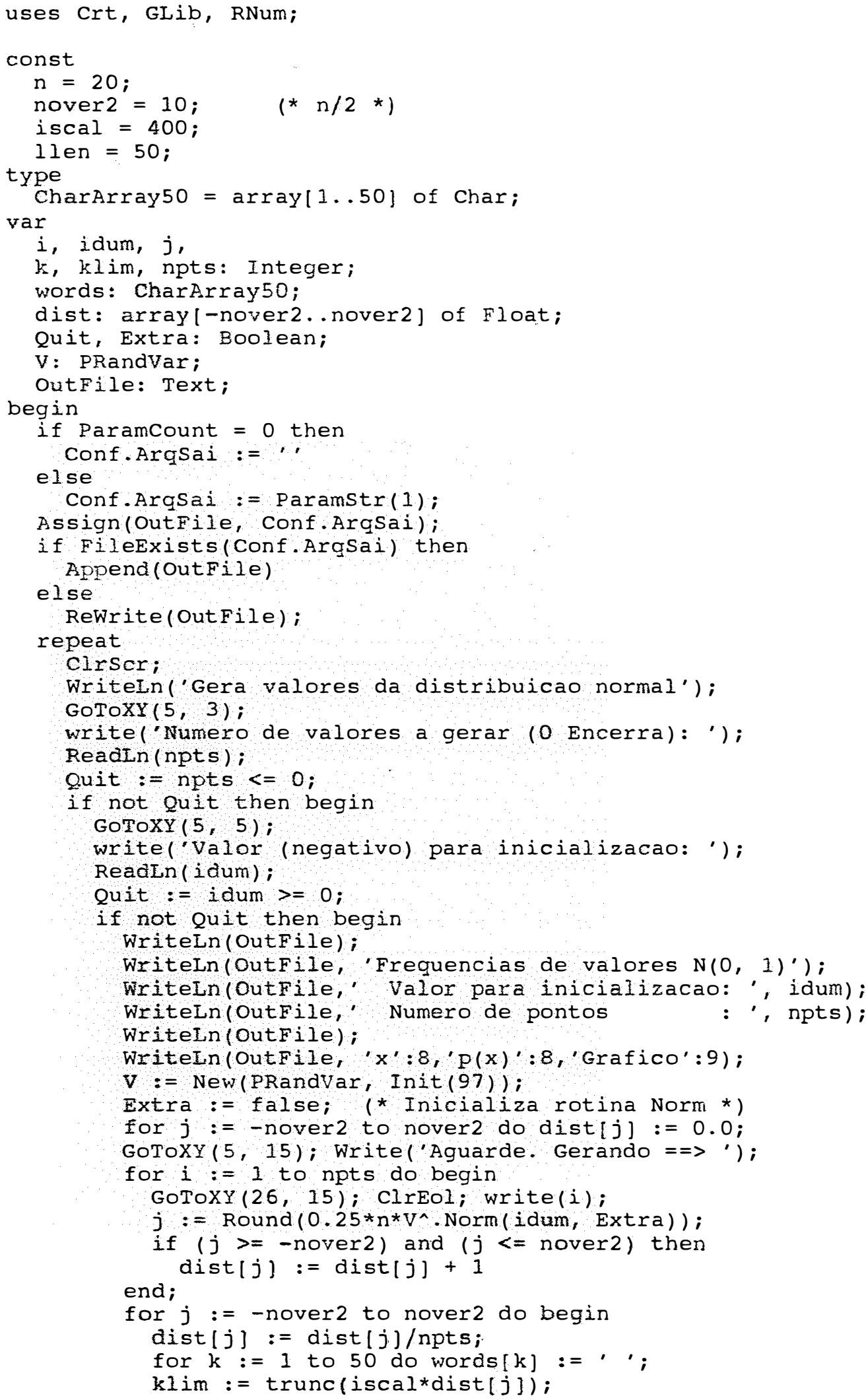


if $\mathrm{klim}>$ lien then $\mathrm{klim}:=$ llen;

for $\mathrm{k}:=1$ to $\mathrm{klim}$ do words $[\mathrm{k}]:=$, $^{\prime}$;

Write (OutFile, $j /\left(0.25^{*} \mathrm{n}\right): 8: 4$, dist $[j]: 8: 4,{ }^{\prime}{ }^{\prime}$ ));

for $k:=1$ to 50 do Write(OutFile, words[k]);

end;

Writeln(OutFile)

Dispose(V, Done);

if Conf.ArgSai $=$ " then begin

Write('Pressione uma tecla para continuar ...');

Readin end;

end;

end;

until Quit;

Close(OutFile);

if Conf.Argsai $<$ ', then end.

Writeln('Saida esta no arquivo: ', conf.ArqSai); 